\title{
On rectifiable measures in Carnot groups: representation
}

\author{
Gioacchino Antonelli ${ }^{1}$. Andrea Merlo ${ }^{2}$
}

Received: 14 April 2021 / Accepted: 16 September 2021 / Published online: 20 November 2021

(c) The Author(s) 2021

\begin{abstract}
This paper deals with the theory of rectifiability in arbitrary Carnot groups, and in particular with the study of the notion of $\mathscr{P}$-rectifiable measure. First, we show that in arbitrary Carnot groups the natural infinitesimal definition of rectifiabile measure, i.e., the definition given in terms of the existence of flat tangent measures, is equivalent to the global definition given in terms of coverings with intrinsically differentiable graphs, i.e., graphs with flat Hausdorff tangents. In general we do not have the latter equivalence if we ask the covering to be made of intrinsically Lipschitz graphs. Second, we show a geometric area formula for the centered Hausdorff measure restricted to intrinsically differentiable graphs in arbitrary Carnot groups. The latter formula extends and strengthens other area formulae obtained in the literature in the context of Carnot groups. As an application, our analysis allows us to prove the intrinsic $C^{1}$-rectifiability of almost all the preimages of a large class of Lipschitz functions between Carnot groups. In particular, from the latter result, we obtain that any geodesic sphere in a Carnot group equipped with an arbitrary left-invariant homogeneous distance is intrinsic $C^{1}$-rectifiable.
\end{abstract}

Mathematics Subject Classification 53C17 · 22E25 $\cdot 28 \mathrm{~A} 75 \cdot 49 \mathrm{Q} 15 \cdot 26 \mathrm{~A} 16$

\section{Introduction}

In the Euclidean setting the notion of rectifiable set, and more in general that of rectifiable measure, can be given in two equivalent ways. Either one could prescribe the infinitesimal behaviour of the measure by saying that it has flat tangent measures almost everywhere, i.e., Hausdorff measures supported on vector subspaces of dimension $k$ or, following a global approach, one could say that the measure is absolutely continuous with respect to the Hausdorff $k$-dimensional measure, and that it is supported on a countable union of $k$-dimensional Lipschitz graphs. In Euclidean spaces the latter two notions are equivalent, pretty wellunderstood and thoroughly studied, see [10,15,38,42].

Communicated by A. Malchiodi.

Gioacchino Antonelli

gioacchino.antonelli@sns.it

1 Scuola Normale Superiore, Piazza dei Cavalieri, 7, Pisa 56126, Italy

2 Université Paris-Saclay, 307 Rue Michel Magat Bâtiment, Orsay 91400, France 
In the last two decades an increasing interest has grown towards the understanding of rectifiability in some specific non-smooth contexts, such as the context of Carnot groups, see Sect. 2 for details. A Carnot group $\mathbb{G}$ is a simply connected nilpotent Lie group, whose Lie algebra is stratified and generated by its first layer. Carnot groups are a generalization of Euclidean spaces, and we remark that (quotients of) Carnot groups arise as the infinitesimal models of sub-Riemannian manifolds. The geometry of a Carnot group, even at an infinitesimal scale, might be very different from the Euclidean one. On every Carnot group we have a natural family of anisotropic dilations $\left\{\delta_{\lambda}\right\}_{\lambda>0}$. We always endow $\mathbb{G}$ with an arbitrary left-invariant homogeneous (with respect to $\left\{\delta_{\lambda}\right\}_{\lambda>0}$ ) distance $d$, and we recall that any two of them are bi-Lipschitz equivalent. We denote $Q$ the Hausdorff dimension of $\mathbb{G}$ with respect to any of such distances.

As shown in the fundamental papers [21,22], in step-2 Carnot groups the reduced boundary of a finite perimeter set can be covered up to $\mathcal{H}^{Q-1}$-negligible sets by countably many intrinsic $C^{1}$-regular hypersurfaces, $C_{\mathrm{H}}^{1}$ hypersurfaces from now on, see Definition 5.2. The positive De Giorgi's rectifiability result in [22] has started an effort to study Geometric Measure Theory in sub-Riemannian Carnot groups, and in particular to study various notion(s) of rectifiability, mostly given following the global approach described at the beginning of this paragraph.

One of the big efforts in this study is trying to understand what is the correct class of building blocks to consider in order to give a satisfactory global definition of rectifiable set, or measure, in the setting of Carnot groups. The first notion of rectifiability that has been proposed and studied is the one which considers as building blocks $C_{\mathrm{H}^{-}}^{1}$-surfaces, as explained above, see [21,23,24,27,35]. Then a notion of intrinsic Lipschitz graph (see Definition 2.12) has been proposed and studied in [17,20], and relations between the notion of intrinsic Lipschitz rectifiability and the notion of $C_{\mathrm{H}}^{1}$-rectifiability have been investigated in [16, 19]. The problem of linking the latter two definitions of rectifiability with the infinitesimal viewpoint was raised in [39] in the setting of Heisenberg groups $\mathbb{H}^{n}$. From the results in [39] one deduces that in $\mathbb{H}^{n}$ the natural infinitesimal notion of rectifiable measure-namely the one given in terms of the existence of flat tangent measures almost everywhere-agrees with the one given in terms of intrinsic Lipschitz graphs in low dimensions, and with the one given in terms of $C_{\mathrm{H}}^{1}$-surfaces in low codimensions. Eventually, it took about ten years to conclude that a Rademacher theorem for intrinsic Lipschitz functions in low codimensions holds in $\mathbb{H}^{n}$, see [43]. As a consequence, at least in $\mathbb{H}^{n}$, the natural infinitesimal definition of rectifiability always agrees with the one given in terms of coverings with intrinsic Lipschitz graphs. An analysis similar to the one of [39] has been pursued in [26] in the setting of homogeneous groups and for measures with horizontal tangents.

Other notions of rectifiability modelled on Lipschitz images of (homogeneous subgroups of) Carnot groups have been proposed by Pauls and Cole-Pauls in [8,41]. An interesting open question asks whether in $\mathbb{H}^{1}$ the notion of rectifiability by means of $C_{\mathrm{H}}^{1}$-hypersurfaces is equivalent to the one of Cole-Pauls given in [8], see [5,11] for some partial results. Nevertheless, in arbitrary Carnot groups, the two notions could be very different, see the results in [2]. A weak notion of rectifiability in terms of building blocks that satisfy some mild cone property has also been recently investigated in [12].

On the other hand, from the infinitesimal viewpoint, a notion that makes sense in arbitrary Carnot groups has been proposed in [40] by the second-named author, namely the notion of $\mathscr{P}$-rectifiable measure, which we recall here. We recall that a subgroup $\mathbb{V}$ of $\mathbb{G}$ is said to be homogeneous if it is closed under the action of the dilations $\left\{\delta_{\lambda}\right\}_{\lambda>0}$. Again we remark that $\mathbb{G}$ is endowed with a left-invariant homogeneous distance $d$. 
Definition 1.1 (P्P-rectifiable measures) Fix a natural number $1 \leq h \leq Q$. A Radon measure $\phi$ on $\mathbb{G}$ is said to be $\mathscr{P}_{h}$-rectifiable if for $\phi$-almost every $x \in \mathbb{G}$ we have

(i) $0<\Theta_{*}^{h}(\phi, x) \leq \Theta^{h, *}(\phi, x)<+\infty$,

(ii) $\operatorname{Tan}_{h}(\phi, x) \subseteq\left\{\lambda \mathcal{S}^{h}\llcorner\mathbb{V}(x): \lambda \geq 0\}\right.$, where $\mathbb{V}(x)$ is a homogeneous subgroup of $\mathbb{G}$ of Hausdorff dimension $h$,

where $\Theta_{*}^{h}(\phi, x)$ and $\Theta^{h, *}(\phi, x)$ are, respectively, the lower and the upper $h$-density of $\phi$ at $x$, see Definition 2.7, $\operatorname{Tan}_{h}(\phi, x)$ is the set of $h$-tangent measures to $\phi$ at $x$, see Definition 2.6, and $\mathcal{S}^{h}$ is the spherical Hausdorff measure of dimension $h$, see Definition 2.4.

In [4] we started to study structure results for the class of $\mathscr{P}$-rectifiable measures, proving a Marstrand-Mattila type rectifiability criterion in the co-normal case [4, Theorem 1.3]. The latter theorem directly leads to the proof of Preiss's theorem in the first Heisenberg group $\mathbb{H}^{1}$ equipped with the Koranyi norm, see [4, Theorem 1.4]. In this paper we complete the study of the notion of $\mathscr{P}$-rectifiable measure, when the tangents are complemented, showing that the notion of $\mathscr{P}$-rectifiability, which is infinitesimal in nature, is equivalent to the global one given in terms of intrinsic differentiable graphs, see Theorem 1.1. We stress that our Theorem 1.1 extends to arbitrary Carnot groups and to all the dimensions the results given in [39].

All in all we conclude that, in Carnot groups, the correct building blocks to consider in order to give a global definition of rectifiability that agrees with the infinitesimal one seem to be intrinsic differentiable graphs. We also provide an area formula for such building blocks, see Theorem 1.3. We stress that, due to the existence of intrinsic Lipschitz graphs that are nowhere intrinsically differentiable, see [28], one cannot give a geometric area formula in the spirit of Theorem 1.3 for arbitrary intrinsic Lipschitz graphs. Nevertheless the area formulae in Theorem 1.2 and Theorem 1.3 extend the area formula given in [27, Theorem 1.1], see the discussion after Theorem 1.3.

We stress that one of the main achievements of this paper is also the rectifiability criterion in Proposition 3.9 which allows to prove the $\mathscr{P}$-rectifiability of almost all the preimages of a large number of Lipschitz functions, see Proposition 1.4, and Corollary 1.5.

Remark Some of the results presented in this paper use results proven in [4, Sections 2-34-6]. We recall the most important ones in the preliminary section of this work, see Sect. 2. During the proofs we give precise references to the results of [4] when we need them.

\subsection{Main results}

We discuss the main contributions of the present paper. We fix $\mathbb{G}$ a Carnot group and we equip it with a left-invariant homogeneous distance. We recall that when we say that a homogeneous subgroup $\mathbb{V}$ admits a complementary subgroup, we mean that there exists a homogeneous subgroup $\mathbb{L}$ such that $\mathbb{G}=\mathbb{V} \cdot \mathbb{L}$ and $\mathbb{V} \cap \mathbb{L}=\{0\}$. The first result of this work is a complete characterization of $\mathscr{P}_{h}$-rectifiable sets with complemented tangents in arbitrary Carnot groups either in terms of the existence of flat $h$-dimensional complemented Preiss's tangents almost everywhere or in terms of a covering property with $h$-dimensional intrinsically differentiable graphs with complemented tangents. We recall that, while $\operatorname{Tan}_{h}(\phi, x)$ captures the behaviour of tangent measures obtained rescaling with the $h$-th power of the scale, see Definition 2.6, the Preiss's tangent Tan $(\phi, x)$, see Definition 2.6, captures the behaviour of all the possible tangent measures, namely

$\operatorname{Tan}(\phi, x):=\left\{v:\right.$ there exist $\left\{c_{i}\right\}$, with $c_{i}>0$, and $\left\{r_{i}\right\}$ with $r_{i} \rightarrow_{i} 0$ such that $\left.c_{i} T_{x, r_{i}} \phi \rightarrow_{i} \nu\right\}$, 
where the convergence of measures is meant in the duality with $C_{c}(\mathbb{G})$, see Definition 2.5. For the reader's convenience we recall here that an intrinsic graph with respect to a splitting $\mathbb{G}=\mathbb{V} \cdot \mathbb{L}$ of the group is said to be intrinsically differentiable at one of its points if the Hausdorff tangent at that point is a homogeneous subgroup, see Definition 3.1 for a precise definition. For the proof of the next statement, see the end of Sect. 3.

Theorem 1.1 Let $\mathbb{G}$ be a Carnot group and fix a natural number $1 \leq h \leq Q$. Let $\Gamma \subseteq \mathbb{G}$ be a Borel set such that $\mathcal{S}^{h}(\Gamma)<+\infty$, where $\mathcal{S}^{h}$ is the h-dimensional spherical Hausdorff measure. Then the following are equivalent

1. $\mathcal{S}^{h}\left\llcorner\Gamma\right.$ is a $\mathscr{P}_{h}$-rectifiable measure with complemented tangents, i.e., a $\mathscr{P}_{h}^{c}$-rectifiable measure, see Definition 2.14,

2. For $\mathcal{S}^{h}\llcorner\Gamma$-almost every $x \in \mathbb{G}$ we have

$$
\begin{gathered}
\operatorname{Tan}\left(\mathcal{S}^{h}\llcorner\Gamma, x)=\left\{\lambda \mathcal{S}^{h}\llcorner\mathbb{V}(x): \lambda>0, \mathbb{V}(x) \text { is acomplemented hom. subgroup of } \mathbb{G}\right.\right. \\
\text { with } \left.\operatorname{dim}_{H} \mathbb{V}(x)=h\right\},
\end{gathered}
$$

3. There exist countably many compact intrinsic Lipschitz graphs $\Gamma_{i}$ that are h-dimensional intrinsically differentiable graphs at $\mathcal{S}^{h}$-almost every $x \in \Gamma_{i}$, that have complemented Hausdorff tangents at $\mathcal{S}^{h}$-almost every $x \in \Gamma_{i}$, and such that

$$
\mathcal{S}^{h}\left(\Gamma \backslash \cup_{i=1}^{+\infty} \Gamma_{i}\right)=0 .
$$

Moreover, denoting with $\mathcal{C}^{h}$ the centered Hausdorff measure of dimension $h$, see Definition 2.4, if any of the previous holds, then $\Theta^{h}\left(\mathcal{C}^{h}\llcorner\Gamma, x)=1\right.$ exists for $\mathcal{C}^{h}\llcorner\Gamma$-almost every $x \in \mathbb{G}$ and

$$
r^{-h}\left(T_{x, r}\right)_{*}\left(\mathcal { C } ^ { h } \llcorner \Gamma ) \rightarrow \mathcal { C } ^ { h } \left\llcorner\mathbb{V}(x), \quad \text { for } \mathcal{C}^{h}\llcorner\Gamma-\text { almost every } x \in \mathbb{G},\right.\right.
$$

where the convergence of measures is meant in the duality with $C_{c}(\mathbb{G})$.

Let us observe that when a Rademacher theorem is available, we can equivalently consider as the building blocks in item 3. of Theorem 1.1 the class of $h$-dimensional intrinsically Lipschitz graphs, without asking anything a priori on the differentiability. Let us recall that a Rademacher theorem is proved in $[16,19]$ in the setting of Carnot groups $\mathbb{G}$ of type $\star$, i.e., a class strictly larger than Carnot groups of step 2, and for maps $\varphi: U \subseteq \mathbb{W} \rightarrow \mathbb{L}$, where $\mathbb{W}$ and $\mathbb{L}$ are complementary subgroups of $\mathbb{G}$, with $\mathbb{L}$ horizontal and one-dimensional. Moreover, with the recent results of [32], the latter codimension-one Rademacher theorem can be extended to the groups of type diamond introduced in [32]. Recently, by making use of the theory of currents, the author of [43] has proved the Rademacher theorem for intrinsically Lipschitz maps between complementary subgroups of any dimension in the Heisenberg groups $\mathbb{H}^{n}$, while in [3] we proved the validity of a Rademacher theorem for co-normal intrinsically Lipschitz graphs.

Nevertheless, Rademacher theorem is now known to be false in arbitrary Carnot groups in a very strict sense, i.e., there exists an $h$-dimensional intrinsically Lipschitz graph in a Carnot group such that at every point of it there exist infinitely many blow-ups and each of these blow-ups is not a homogeneous subgroup, see [28, Theorem 1.1]. This latter result implies that in general in item 3. of Theorem 1.1 one cannot equivalently consider as building blocks of a locally well-behaved definition of rectifiable sets the family of $h$-dimensional intrinsically Lipschitz graphs. So, in some sense, the result of Theorem 1.1 is sharp also in view of the negative result of [28].

Let us further notice that we do not consider in this work the relations between the three items in Theorem 1.1 and the existence of an approximate tangent in the sense of [38, 
Definition 15.17] (cf. [39, Definition 3.7]). This relation will be target of future investigations. All in all, taking into account that $\mathcal{S}^{h}\left\llcorner\Gamma\right.$ is $\mathscr{P}_{h}^{c}$-rectifiable with co-horizontal tangents if and only if $\Gamma$ is $C_{H}^{1}\left(\mathbb{G}, \mathbb{R}^{Q-h}\right)$-rectifiable, see Definition 5.3 and Corollary 5.3, our result in Theorem 1.1 extends and strengthens [39, (i) $\Leftrightarrow($ ii $) \Leftrightarrow($ iv $) \Leftrightarrow(v)$ of Theorem 3.15]. Notice also that in the previous chain of equivalences, we can also drop the assumption on the lower density in [39, (iv),(v)]. Moreover, taking into account the Rademacher theorem of [3] in the co-normal case, our result in Theorem 1.1 extends $[39$, (i) $\Leftrightarrow(\mathrm{ii}) \Leftrightarrow(\mathrm{iv}) \Leftrightarrow(\mathrm{v})$ of Theorem 3.14] as well. Let us recall, for the reader's convenience, that [39, Theorem 3.15] deals with the characterization of co-horizontal rectifiability in the Heisenberg groups $\mathbb{H}^{n}$, while [39, Theorem 3.14] deals with the characterization of horizontal rectifiability in the Heisenberg groups $\mathbb{H}^{n}$.

Let us final notice that we stated our result in Theorem 1.1 for measures of the form $\mathcal{S}^{h}\left\llcorner\Gamma\right.$, but we could also give a version of it for Radon measures with $\Theta^{h, *}(\phi, x)<+\infty$ for $\phi$-almost every $x \in \mathbb{G}$, after having proven the analogue of Theorem 3.7 for measures.

The second result of the work is an area formula for intrinsic Lipschitz graphs that are in addition $\mathscr{P}_{h}^{c}$-rectifiable. The proof of the following statement is at the end of Sect. 4.

Theorem 1.2 Let $\mathbb{V}, \mathbb{L}$ be homogeneous complementary subgroups of a Carnot group $\mathbb{G}$ such that $h:=\operatorname{dim}_{H} \mathbb{V}$. Let $\Gamma$ be the graph of an intrinsic Lipschitz map $\varphi: A \subseteq \mathbb{V} \rightarrow \mathbb{L}$ (see Definition 2.12), with A Borel, such that $\mathcal{S}^{h}\left\llcorner\Gamma\right.$ is $\mathscr{P}_{h}^{c}$-rectifiable with tangent measures $\mathcal{S}^{h}\llcorner\Gamma$-almost everywhere supported on homogeneous subgroups complemented by $\mathbb{L}$. Then, for every Borel function $\psi: \Gamma \rightarrow[0,+\infty)$ the following area formula holds

$$
\int_{\Gamma} \psi d \mathcal{C}^{h}\left\llcorner\Gamma=\int_{A} \psi(a \cdot \varphi(a)) \mathcal{A}(\mathbb{V}(a \cdot \varphi(a))) d \mathcal{C}^{h}\llcorner\mathbb{V},\right.
$$

where $\mathcal{C}^{h}$ is the centered Hausdorff measure, see Definition 2.4, $\mathbb{V}(a \cdot \varphi(a))$ is the tangent on which it is supported the tangent measure of $\mathcal{S}^{h}\llcorner\Gamma$ at the point $a \cdot \varphi(a) \in \Gamma$, and $\mathcal{A}(\cdot)$ is the centered area factor defined with respect to the splitting $\mathbb{G}=\mathbb{V} \cdot \mathbb{L}$, see Definition 4.1.

A consequence of the previous result is the following one, which is an area formula for intrinsic Lipschitz graphs that are also intrinsically differentiable. The proof of the following statement can be found at the end of Sect. 4.

Theorem 1.3 Let $\mathbb{V}, \mathbb{L}$ be homogeneous complementary subgroups of a Carnot group $\mathbb{G}$ such that $h=\operatorname{dim}_{H} \mathbb{V}$. Let $\Gamma$ be the graph of an intrinsic Lipschitz map $\varphi: A \subseteq \mathbb{V} \rightarrow \mathbb{L}$, with $A$ Borel. Let us assume $\Gamma$ is an intrinsically differentiable graph (see Definition 3.1) at $\mathcal{S}^{h}$-almost every $x \in \Gamma$ and let us assume that the Hausdorff tangent $\mathbb{V}(x)$ of $\Gamma$ at $x$ is complemented by $\mathbb{L}$ at $\mathcal{S}^{h}$-almost every $x \in \Gamma$. Then, for every Borel function $\psi: \Gamma \rightarrow[0,+\infty)$, the area formula in (1) holds.

Let us remark that, taking into account that $\Theta^{h}\left(\mathcal{C}^{h}\llcorner\Gamma, x)=1\right.$ for $\mathcal{C}^{h}\llcorner\Gamma$-almost every $x \in \mathbb{G}$, see Theorem 1.1, and considering the result in [27, Corollary 3.6] one can show that (1) extends and strengthens the area formula of [27, Theorem 1.1]. Indeed, the graph of a $C_{\mathbb{W}, \mathbb{V}}^{1}$ function as in the statement of [27, Theorem 1.1] is a $\mathscr{P}_{h}^{c}$-rectifiable set, see [4, Proposition 6.2]. Moreover, we stress that Theorem 1.2 strictly strengthens [27, Theorem 1.1] for two reasons: there exists natural examples of graphs that are $\mathscr{P}_{h}$-rectifiable but not $C_{\mathrm{H}^{-}}^{1}$-rectifiable, see [4, Remark 6.3], and moreover in our result the map $\varphi$ does not need to be defined on an open set but just on a Borel set.

Let us notice that the area formula in Theorem 1.3 is geometric. It roughly asserts that when an intrinsic Lipschitz graph over the split $\mathbb{V} \cdot \mathbb{L}$ has almost everywhere a flat Hausdorff tangent 
complemented by $\mathbb{L}$, then the area of this graph can be obtained integrating on $\mathbb{V}$ a geometric area factor. With geometric we mean that the factor only depends on the tangent. Let us stress that when a Rademacher theorem is available, one can remove the hypothesis about the intrinsic differentiability in Theorem 1.3. Nevertheless, as discussed above, a Rademacher theorem might not hold in arbitrary Carnot groups, see [28].

Let us point out that in the literature one can find many more analytic area formulae in Carnot groups, i.e., in which the area element is expressed in terms of properly defined intrinsic derivatives of the map $\varphi$. This is the case of [9, Theorem 1.1 and Theorem 1.2] for low-codimensional $C_{\mathrm{H}^{-}}^{1}$-surfaces in Heisenberg groups (cf. also [20, Theorem 2]), which has been extended to intrinsic Lipschitz low-codimensional surfaces in [43, Theorem 1.3] (cf. also [7, Theorem 1.6]); and of [1, Proposition 1.8] for one-codimensional $C_{\mathrm{H}^{-}}^{1}$ graphs in arbitrary Carnot groups. These formulas could be derived from Theorem 1.3 explicitly writing the area element in terms of the intrinsic derivatives of the parametrisation map $\varphi$. Other geometric area formulae for Euclidean $C^{1}$ or $C^{1,1}$-submanifolds in Carnot groups have been investigated in $[34,36,37]$. Let us remark that our point of view is intrinsic while on the contrary the works $[34,36,37]$ investigate Euclidean-regular manifolds. The results in [37, Theorem 1.1 and Theorem 1.2], [36, Theorem 1.1], and [34, Theorem 1.1 and Theorem 1.2] roughly assert that whenever a point of a Euclidean-regular submanifold is sufficiently nice, then the intrinsic blow-up at that point exists and it is a homogeneous subgroup; and as a consequence also the density - of the correct dimension - of the (Euclidean) volume measure of the submanifold exists at that point. Then what one notices is that in a lot of cases the nice points are almost all-with respect to the intrinsic Hausdorff measure of the correct dimension - the points of the submanifold, cf. [36, Theorem 1.2]. These latter results have to be compared with our Proposition 3.9 in which we prove that having almost everywhere an intrinsic complemented blow-up implies the existence of the density of the Hausdorff measure. Let us notice that when a negligibility theorem, a blow-up theorem, and the existence of the density hold in the sense of [34,36,37] discussed above for a Euclideanregular submanifold $\Sigma$, then one gets that $\operatorname{Tan}_{h}\left(\mathcal{S}^{h}\llcorner\Sigma, x) \subseteq\left\{\lambda \mathcal{S}^{h}\llcorner\mathbb{V}(x): \lambda>0\}\right.\right.$ holds for $\mathcal{S}^{h}$-almost every $x \in \Sigma$, where $h$ is the Hausdorff dimension of $\Sigma$, and where $\mathbb{V}(x)$ is a homogeneous subgroup. This last observation easily follows arguing as in the last lines of the proof of Proposition 3.9. As a result, when a negligibility theorem, a blow-up theorem, and the existence of the density hold in the sense of [34,36,37] for a Euclidean-regular submanifold $\Sigma$, one gets that $\mathcal{S}^{h}\left\llcorner\Sigma\right.$ is a $\mathscr{P}_{h}$-rectifiable measure.

Let us finally notice that the area formula in Theorem 1.3 is a formula for the building blocks for the global notion of rectifiability in item 3. of Theorem 1.1. Thus, by localization, one obtains from Theorem 1.3 and Theorem 1.1, an area formula for arbitrary $\mathscr{P}_{h}^{c}$-rectifiable measures, see Corollary 4.7.

The third result of the work is a rectifiability result for the level sets of Lipschitz functions between Carnot groups. For the proof of the following statement, we refer the reader to Proposition 5.1.

Proposition 1.4 Let B be a Borel set in $\mathbb{G}$ and suppose $\mathbb{H}$ is a Carnot group of homogeneous dimension $Q^{\prime}$ with $Q \geq Q^{\prime}$. Let $f: B \subseteq \mathbb{G} \rightarrow \mathbb{H}$ be a Lipschitz map such that

$$
\begin{aligned}
& \operatorname{Ker}(d f(x)) \text { is a complemented homogeneous subgroup of } \mathbb{G} \text { for } \mathcal{S}^{Q} \\
& \quad \text {-almost every } x \in\{z \in B: d f(z) \text { exists surjective }\}
\end{aligned}
$$

where $d f(x)$ is the Pansu differential that exists for $\mathcal{S}^{Q}$-almost every $x \in \mathbb{G}$, see Definition 5.1. Then, for $\mathcal{S}^{Q^{\prime}}$-almost every $y \in f(B)$, the measure $\mathcal{S}^{Q-Q^{\prime}} f^{-1}(y)$ is $\mathscr{P}_{Q-Q^{\prime}}^{c}$-rectifiable 
in $\mathbb{G}$ and

$$
\begin{aligned}
& \operatorname{Tan}_{Q-Q^{\prime}}\left(\mathcal { S } ^ { Q - Q ^ { \prime } } \llcorner f ^ { - 1 } ( y ) , x ) \subseteq \left\{\lambda \mathcal{S}^{Q-Q^{\prime}}\llcorner\operatorname{Ker}(d f(x)): \lambda>0\}, \quad \text { forS } \mathcal{S}^{Q-Q^{\prime}}\right.\right. \\
& \quad \text {-almost everyx } \in f^{-1}(y) .
\end{aligned}
$$

As an immediate consequence of the previous result we have the following corollary, which is worth pointing out explicitly, since, even if simple to state, it seems to be not present in the literature up to the authors' knowledge. For a proof, see Corollary 5.4. We remark that a more general statement, in the co-horizontal case, is still true and can be found in Corollary 5.2 .

Corollary 1.5 Suppose $f: B \subseteq \mathbb{G} \rightarrow \mathbb{R}$ is a Lipschitz map, where $B$ is a Borel set. Then, for $\mathcal{S}^{1}$-almost every $y \in f(B)$ the set $f^{-1}(y)$ is $C_{\mathrm{H}}^{1}$-rectifiable. In particular, for every $r>0$ and $x \in \mathbb{G}$, every geodesic sphere $\partial B_{r}(x)$ is $C_{\mathrm{H}^{1}}^{1}$-rectifiable.

Notice that the last part of the previous result comes from the first applied to the distance function from $x$, and the observation that, by dilating, once $\partial B_{r}(x)$ is $C_{\mathrm{H}}^{1}$-rectifiable for one radius $r>0$, then it is $C_{\mathrm{H}}^{1}$-rectifiable for every radius $r>0$. The previous corollary should be compared with [44, Theorem 3.2], where an intrinsic Lipschitz rectifiability for Lipschitz surfaces is proved in CC-spaces. Notice, however, that nowadayas it is not known whether, in codimension-one, intrinsic Lipschitz rectifiability and $C_{\mathrm{H}}^{1}$-rectifiability are equivalent in arbitrary Carnot groups; while being intrinsic Lipschitz rectifiable is weaker than being $C_{\mathrm{H}}^{1}$-rectifiable. Nevertheless, our previous corollary provides the $C_{\mathrm{H}}^{1}$-rectifiability of all the spheres in every Carnot group. It is however interesting to point out how the Euclidean rectifiability of the geodesic spheres in Carnot groups is still an intriguing open question in general, and it is related to asymptotic volume expansion in nilpotent Lie groups [6,31].

Let us remark that the previous results in Proposition 1.4 and Corollary 1.5 follow from the rectifiability criterion in Proposition 3.9. It is worth pointing out that, given a Lipschitz function $f: B \subseteq \mathbb{G} \rightarrow \mathbb{R}$, for every $y \in f(B)$, the set $\{f \leq y\}$ is of locally finite perimeter in $\mathbb{G}$, see, e.g., [13, Theorem 2.40]. Hence, taking into account Corollary 1.5, we deduce the following consequence: $\mathcal{S}^{1}$-almost all the sublevel sets of real-valued Lipschitz functions defined on Borel subsets of Carnot groups are examples of sets of locally finite perimeter whose boundary is $C_{\mathrm{H}^{-}}^{1}$-rectifiable—namely De Giorgi's rectifiability Theorem holds for such sets.

Let us finally stress that the previous results in Proposition 1.4 and Corollary 1.5 open the way to proving slicing theorems and coarea formulae for $\mathscr{P}$-rectifiable and Lipschitz slicing functions. This will be target of future investigations.

Let us briefly comment on the proof of Theorem 1.1. For what concerns the implications 1 . $\Rightarrow 2$., and 1. $\Rightarrow 3$., the first is just a matter of routine argument, see [38, Remark 14.4(3)], and the second is a consequence of [4, Theorem 1.8]. The main new contributions of this paper, which lead to the equivalence in Theorem 1.1, are the implications 2 . $\Rightarrow 1$., and 3 . $\Rightarrow$ 1., both of them non-trivial.

For what concerns the implication 2. $\Rightarrow$ 1., we first use that the hypothesis of flat Preiss's tangents allows to conclude that $\Gamma$ is $\mathcal{S}^{h}$-almost everywhere covered by countably many $h$-dimensional graphs $\Gamma_{i}$ of intrinsically Lipschitz functions, namely $\mathcal{S}^{h}\left(\Gamma \backslash \cup_{i=1}^{+\infty} \Gamma_{i}\right)=0$, see Proposition 2.26. Hence we exploit the general fact, that dates back to Preiss's paper (cf. [42, Corollary 2.7]), that a measure with a compact-based tangent at a point is asymptotically doubling at that point. Joining the latter two observations, we deduce that, for every $i$, the measure $\mathcal{S}^{h}\left\llcorner\Gamma_{i}\right.$ is asymptotically doubling, and then this enables us to prove that $\Gamma_{i}$ has big projections on the plane over which $\Gamma_{i}$ is a graph, see Proposition 3.3. The latter proposition 
is just a straightforward empowerment of our result already proved in [4, Proposition 4.6]. Finally, the big projections property of Proposition 3.3 allows us to conclude that the $h$-lower density $\Theta_{*}^{h}\left(\mathcal{S}^{h}\left\llcorner\Gamma_{i}, \cdot\right)\right.$ is positive $\mathcal{S}^{h}\left\llcorner\Gamma_{i}\right.$ almost everywhere, see Proposition 3.6. Hence, the proof of the implication 2 . $\Rightarrow 1$. is concluded since we can argue, by exploiting Lebesgue differentiation theorem, that $\Theta_{*}^{h}\left(\mathcal{S}^{h}\llcorner\Gamma, \cdot)\right.$ is positive $\mathcal{S}^{h}\llcorner\Gamma$-almost everywhere, which was the non-trivial missing information to prove 1 . Let us notice that in 2 . we are not requiring anything a priori on the positivity of the $h$-lower density of $\mathcal{S}^{h}\llcorner\Gamma$, otherwise the implication 2 . $\Rightarrow 1$. would have been trivial. Nevertheless, we deduce the positivity of the $h$-lower density from the fact that the tangents are flat and complemented as we discussed above.

The proof of the implication 3. $\Rightarrow 1$. relies on the fact that an arbitrary $h$-dimensional (almost everywhere) intrinsically differentiable graph $\Gamma$ with complemented Hausdorff tangents has the property that $\mathcal{S}^{h}\left\llcorner\Gamma\right.$ is $\mathscr{P}_{h}^{c}$-rectifiable. This is exactly the content of Proposition 3.9. In order to prove the latter proposition, we show that when we have an arbitrary $h$ dimensional (almost everywhere) intrinsically differentiable graph $\Gamma$ with complemented Hausdorff tangents, at $\left(\mathcal{S}^{h}\llcorner\Gamma\right.$-almost) every point we have that the graph $\Gamma$ is, at arbitrarily small scales, contained in a cone with arbitrarily small opening and with basis the Hausdorff tangent at that point. This observation enables us to perform a covering argument and to show directly that $\Theta^{h}\left(\mathcal{C}^{h}\llcorner\Gamma, \cdot)=1\right.$ at $\mathcal{C}^{h}\left\llcorner\Gamma\right.$-almost every point. Then the fact that $\mathcal{C}^{h}\llcorner\Gamma$, and hence $\mathcal{S}^{h}\left\llcorner\Gamma\right.$, is $\mathscr{P}_{h}^{c}$-rectifiable is reached by using a classical argument, see Proposition 2.3 and Proposition 2.4. Let us notice that in Proposition 3.9 it is essential to work with the centered Hausdorff measure $\mathcal{C}^{h}\llcorner\Gamma$, since we consider coverings with balls centered on $\Gamma$. It is also worth noticing that $\mathcal{S}^{h}\left\llcorner\Gamma\right.$ and $\mathcal{C}^{h}\llcorner\Gamma$ are mutually absolutely continuous so any rectifiability property for one measure is transferred to the other by means of Lebesgue differentiation theorem and the locality of tangents.

The final part of the statement in Theorem 1.1 is a consequence of the fact that the $h$-density of $\mathcal{C}^{h}\left\llcorner\Gamma\right.$ is 1 as a consequence of the previous reasoning, and the fact that $\mathcal{C}^{h}\llcorner\mathbb{V}(B(0,1))=1$ for every homogeneous subgroup $\mathbb{V}$ of Hausdorff dimension $h$, see Lemma 2.1 .

Let us briefly comment on the proof of Theorem 1.2. The strategy of the proof is similar to the one in [27] and it is based on a continuity property of the volumes through a blow-up procedure, see Proposition 4.4. Nevertheless, in order to prove Proposition 4.4, one needs to face some delicate technical problems due to the fact that the map $\varphi$ is not necessarily defined on an open set, but just on a Borel set. Hence, one needs to argue directly on the graph by using a Vitali-type differentiation theorem, see Proposition 4.3, and a new delicate estimate on the volumes of the projections of balls in $\Gamma$, see Proposition 3.8.

\section{Preliminaries}

\subsection{Carnot groups}

In this subsection we briefly introduce some notations on Carnot groups that we will extensively use throughout the paper. For a detailed account on Carnot groups we refer to [29].

A Carnot group $\mathbb{G}$ of step $\kappa$ is a connected and simply connected Lie group whose Lie algebra $\mathfrak{g}$ admits a stratification $\mathfrak{g}=V_{1} \oplus V_{2} \oplus \cdots \oplus V_{\kappa}$. We say that $V_{1} \oplus V_{2} \oplus \cdots \oplus V_{\kappa}$ is a stratification of $\mathfrak{g}$ if $\mathfrak{g}=V_{1} \oplus V_{2} \oplus \cdots \oplus V_{\kappa}$,

$$
\left[V_{1}, V_{i}\right]=V_{i+1}, \quad \text { for any } i=1, \ldots, \kappa-1, \quad \text { and }\left[V_{1}, V_{\kappa}\right]=\{0\}
$$


where $[A, B]:=\operatorname{span}\{[a, b]: a \in A, b \in B\}$. We call $V_{1}$ the horizontal layer of $\mathbb{G}$. We denote by $n$ the topological dimension of $\mathfrak{g}$, by $n_{j}$ the dimension of $V_{j}$ for every $j=1, \ldots, \kappa$. Furthermore, we define $\pi_{i}: \mathbb{G} \rightarrow V_{i}$ to be the projection maps on the $i$-th strata. We will often shorten the notation to $v_{i}:=\pi_{i} v$.

The exponential map exp : $\mathfrak{g} \rightarrow \mathbb{G}$ is a global diffeomorphism from $\mathfrak{g}$ to $\mathbb{G}$. Hence, if we choose a basis $\left\{X_{1}, \ldots, X_{n}\right\}$ of $\mathfrak{g}$, any $p \in \mathbb{G}$ can be written in a unique way as $p=\exp \left(p_{1} X_{1}+\cdots+p_{n} X_{n}\right)$. This means that we can identify $p \in \mathbb{G}$ with the $n$-tuple $\left(p_{1}, \ldots, p_{n}\right) \in \mathbb{R}^{n}$ and the group $\mathbb{G}$ itself with $\mathbb{R}^{n}$ endowed with $\cdot$ the group operation determined by the Baker-Campbell-Hausdorff formula. From now on, we will always assume that $\mathbb{G}=\left(\mathbb{R}^{n}, \cdot\right)$ and, as a consequence, that the exponential map exp acts as the identity.

The stratificaton of $\mathfrak{g}$ carries with it a natural family of dilations $\delta_{\lambda}: \mathfrak{g} \rightarrow \mathfrak{g}$, that are Lie algebra automorphisms of $\mathfrak{g}$ and are defined by

$$
\delta_{\lambda}\left(v_{1}, \ldots, v_{\kappa}\right):=\left(\lambda v_{1}, \lambda^{2} v_{2}, \ldots, \lambda^{\kappa} v_{\kappa}\right), \text { for any } \lambda>0,
$$

where $v_{i} \in V_{i}$. The stratification of the Lie algebra $\mathfrak{g}$ naturally induces a gradation on each of its homogeneous Lie sub-algebras $\mathfrak{h}$, i.e., a sub-algebra that is $\delta_{\lambda}$-invariant for any $\lambda>0$, that is

$$
\mathfrak{h}=V_{1} \cap \mathfrak{h} \oplus \ldots \oplus V_{\kappa} \cap \mathfrak{h} .
$$

We say that $\mathfrak{h}=W_{1} \oplus \cdots \oplus W_{\kappa}$ is a gradation of $\mathfrak{h}$ if $\left[W_{i}, W_{j}\right] \subseteq W_{i+j}$ for every $1 \leq i, j \leq \kappa$, where we mean that $W_{\ell}:=\{0\}$ for every $\ell>\kappa$. Since the exponential map acts as the identity, the Lie algebra automorphisms $\delta_{\lambda}$ are also group automorphisms of $\mathbb{G}$.

Definition 2.1 (Homogeneous subgroups) A subgroup $\mathbb{V}$ of $\mathbb{G}$ is said to be homogeneous if it is a Lie subgroup of $\mathbb{G}$ that is invariant under the dilations $\delta_{\lambda}$.

We recall the following basic terminology: a horizontal subgroup of a Carnot group $\mathbb{G}$ is a homogeneous subgroup of it that is contained in $\exp \left(V_{1}\right)$; a Carnot subgroup $\mathbb{W}=\exp (\mathfrak{h})$ of a Carnot group $\mathbb{G}$ is a homogeneous subgroup of it such that the first layer $V_{1} \cap \mathfrak{h}$ of the grading of $\mathfrak{h}$ inherited from the stratification of $\mathfrak{g}$ is the first layer of a stratification of $\mathfrak{h}$.

Homogeneous Lie subgroups of $\mathbb{G}$ are in bijective correspondence through exp with the Lie sub-algebras of $\mathfrak{g}$ that are invariant under the dilations $\delta_{\lambda}$. For any Lie algebra $\mathfrak{h}$ with gradation $\mathfrak{h}=W_{1} \oplus \ldots \oplus W_{\kappa}$, we define its homogeneous dimension as

$$
\operatorname{dim}_{\text {hom }}(\mathfrak{h}):=\sum_{i=1}^{\kappa} i \cdot \operatorname{dim}\left(W_{i}\right) .
$$

Thanks to (2) we infer that, if $\mathfrak{h}$ is a homogeneous Lie sub-algebra of $\mathfrak{g}$, we have $\operatorname{dim}_{\text {hom }}(\mathfrak{h}):=$ $\sum_{i=1}^{\kappa} i \cdot \operatorname{dim}\left(\mathfrak{h} \cap V_{i}\right)$. It is well-known that the Hausdorff dimension (for a definition of Hausdorff dimension see for instance [38, Definition 4.8]) of a graded Lie group $\mathbb{G}$ with respect to a left-invariant homogeneous distance coincides with the homogeneous dimension of its Lie algebra. For a reference for the latter statement, see [30, Theorem 4.4]. From now on, if not otherwise stated, $\mathbb{G}$ will be a fixed Carnot group.

For any $p \in \mathbb{G}$, we define the left translation $\tau_{p}: \mathbb{G} \rightarrow \mathbb{G}$ as

$$
q \mapsto \tau_{p} q:=p \cdot q .
$$

As already remarked above, the group operation - is determined by the Campbell-Hausdorff formula, and it has the form (see [21, Proposition 2.1])

$$
p \cdot q=p+q+\mathscr{Q}(p, q), \text { for all } p, q \in \mathbb{R}^{n},
$$


where $\mathscr{Q}=\left(\mathscr{Q}_{1}, \ldots, \mathscr{Q}_{\kappa}\right): \mathbb{R}^{n} \times \mathbb{R}^{n} \rightarrow V_{1} \oplus \ldots \oplus V_{\kappa}$, and the $\mathscr{Q}_{i}$ 's have the following properties. For any $i=1, \ldots \kappa$ and any $p, q \in \mathbb{G}$ we have

(i) $\mathscr{Q}_{i}\left(\delta_{\lambda} p, \delta_{\lambda} q\right)=\lambda^{i} \mathscr{Q}_{i}(p, q)$,

(ii) $\mathscr{Q}_{i}(p, q)=-\mathscr{Q}_{i}(-q,-p)$,

(iii) $\mathscr{Q}_{1}=0$ and $\mathscr{Q}_{i}(p, q)=\mathscr{Q}_{i}\left(p_{1}, \ldots, p_{i-1}, q_{1}, \ldots, q_{i-1}\right)$.

Thus, we can represent the product · as

$$
\begin{aligned}
p \cdot q= & \left(p_{1}+q_{1}, p_{2}+q_{2}+\mathscr{Q}_{2}\left(p_{1}, q_{1}\right), \ldots, p_{\kappa}+q_{\kappa}\right. \\
& \left.+\mathscr{Q}_{\kappa}\left(p_{1}, \ldots, p_{\kappa-1}, q_{1}, \ldots, q_{\kappa-1}\right)\right) .
\end{aligned}
$$

Definition 2.2 (Homogeneous left-invariant distance and norm) A metric $d: \mathbb{G} \times \mathbb{G} \rightarrow \mathbb{R}$ is said to be homogeneous and left-invariant if for any $x, y \in \mathbb{G}$ we have, respectively

(i) $d\left(\delta_{\lambda} x, \delta_{\lambda} y\right)=\lambda d(x, y)$ for any $\lambda>0$,

(ii) $d\left(\tau_{z} x, \tau_{z} y\right)=d(x, y)$ for any $z \in \mathbb{G}$.

Given a homogeneous left-invariant distance, its associated norm is defined by $\|g\|_{d}:=$ $d(g, 0)$, for every $g \in \mathbb{G}$, where 0 is the identity element of $\mathbb{G}$.

Given a homogeneous left-invariant distance $d$ on $\mathbb{G}$, for every $x \in \mathbb{G}$ and every $E \subseteq \mathbb{G}$ we define $\operatorname{dist}(x, E):=\inf \{d(x, y): y \in E\}$.

Throughout the paper we will always endow, if not otherwise stated, the group $\mathbb{G}$ with an arbitrary homogeneous and left-invariant metric. We will denote such a distance with $d$. We remark that two homogeneous left-invariant distances on a Carnot group are always bi-Lipschitz equivalent.

Definition 2.3 (Metric balls and tubular neighbourhoods) Suppose a homogeneous and leftinvariant metric $d$ has been fixed on $\mathbb{G}$. Then, we define $U_{d}(x, r):=\{z \in \mathbb{G}: d(x, z)<r\}$ to be the open metric ball relative to the distance $d$ centred at $x$ at radius $r>0$. The closed ball will be denoted with $B_{d}(x, r):=\{z \in \mathbb{G}: d(x, z) \leq r\}$. Moreover, for a subset $E \subseteq \mathbb{G}$ and $r>0$, we denote with $B_{d}(E, r):=\{z \in \mathbb{G}: \operatorname{dist}(z, E) \leq r\}$ the closed $r$-tubular neighborhood of $E$ and with $U_{d}(E, r):=\{z \in \mathbb{G}: \operatorname{dist}(z, E)<r\}$ the open $r$-tubular neighborhood of $E$. When the metric $d$ is understood, we will tacitly drop the dependence on the metric in the notation.

Definition 2.4 (Hausdorff Measures) Let $d$ be a homogeneous and left-invariant metric on $\mathbb{G}$ and $A \subseteq \mathbb{G}$ be a Borel set. For any $0 \leq h \leq \mathcal{Q}$ and $\delta>0$, define

$$
\begin{aligned}
& \mathscr{C}_{d, \delta}^{h}(A):=\inf \left\{\sum_{j=1}^{\infty} r_{j}^{h}: A \subseteq \bigcup_{j=1}^{\infty} B_{d}\left(x_{j}, r_{j}\right), r_{j} \leq \delta \text { and } x_{j} \in A\right\}, \\
& \mathscr{S}_{d, \delta}^{h}(A):=\inf \left\{\sum_{j=1}^{\infty} r_{j}^{h}: A \subseteq \bigcup_{j=1}^{\infty} B_{d}\left(x_{j}, r_{j}\right), r_{j} \leq \delta\right\},
\end{aligned}
$$

and $\mathscr{S}_{\delta, E}^{h}(\emptyset):=0=: \mathscr{C}_{\delta}^{h}(\emptyset)$. Eventually, we let

$$
\begin{aligned}
\mathcal{C}_{d}^{h}(A):=\sup _{B \subseteq A} \sup _{\delta>0} \mathscr{C}_{d, \delta}^{h}(B) & =\sup _{B \subseteq A} \mathcal{C}_{d, 0}^{h}(B) \text { be the centred spherical Hausdorff measure, } \\
\mathcal{S}_{d}^{h}(A) & :=\sup _{\delta>0} \mathscr{S}_{d, \delta}^{h}(A) \text { be the spherical Hausdorff measure. }
\end{aligned}
$$


We stress that $\mathcal{C}_{d}^{h}$ is an outer measure, and thus it defines a Borel regular measure, see [14, Proposition 4.1], and that the measures $\mathcal{S}_{d}^{h}, \mathcal{H}_{d}^{h}, \mathcal{C}_{d}^{h}$ are all equivalent measures, see [15, Section 2.10.2] and [14, Proposition 4.2]. When the metric $d$ is understood, we will tacitly drop the dependence on the metric in the notation.

We recall here the following result that has been proved in [4, item (iii) of Proposition 2.11], and that will often be used in the paper.

Lemma 2.1 Let $\mathbb{V}$ be a homogeneous subgroup of a Carnot group $\mathbb{G}$ endowed with a leftinvariant homogeneous distance $d$. Let us call h the homogeneous dimension of $\mathbb{V}$. Hence

$$
\mathcal{C}_{d}^{h}\left(B_{d}(x, r) \cap \mathbb{V}\right)=r^{h}
$$

for every $x \in \mathbb{V}$ and any $r>0$.

\subsection{Densities and tangents of Radon measures}

Throughout the rest of the paper we will always assume that $\mathbb{G}$ is a fixed Carnot group endowed with a left-invariant homogeneous distance $d$. The homogeneous, and thus Hausdorff, dimension with respect to $d$ will be denoted with $Q$. Furthermore, as discussed in the previous subsection, we will assume without loss of generality that $\mathbb{G}$ coincides with $\mathbb{R}^{n}$ endowed with the product induced by the Baker-Campbell-Hausdorff formula.

Definition 2.5 (Weak convergence of measures) Given a family $\left\{\phi_{i}\right\}_{i \in \mathbb{N}}$ of Radon measures on $\mathbb{G}$ we say that the sequence $\phi_{i}$ weakly* converges to a Radon measure $\phi$, and we write $\phi_{i} \rightarrow \phi$, if

$$
\int f d \phi_{i} \rightarrow \int f d \phi \quad \text { for any } f \in C_{c}(\mathbb{G})
$$

where $C_{c}(\mathbb{G})$ is the space of compactly supported functions on $\mathbb{G}$.

Definition 2.6 (Tangent measures) Let $\phi$ be a Radon measure on $\mathbb{G}$. For any $x \in \mathbb{G}$ and any $r>0$ we define the measure

$$
T_{x, r} \phi(E):=\phi\left(x \cdot \delta_{r}(E)\right), \quad \text { for any Borel set } E .
$$

Furthermore, we define $\operatorname{Tan}(\phi, x)$, the tangent measures to $\phi$ at $x$, to be the collection of the non-null Radon measures $v$ for which there is a sequence $\left\{r_{i}\right\}_{i \in \mathbb{N}}$, with $r_{i} \rightarrow 0$, and a sequence $\left\{c_{i}\right\}_{i \in \mathbb{N}}$, with $c_{i}>0$, such that

$$
c_{i} T_{x, r_{i}} \phi \rightarrow v .
$$

Moreover, we define $\operatorname{Tan}_{h}(\phi, x)$, the $h$-tangent measures to $\phi$ at $x$, to be the collection of Radon measures $v$ for which there is a sequence $\left\{r_{i}\right\}_{i \in \mathbb{N}}$, with $r_{i} \rightarrow 0$, such that

$$
r_{i}^{-h} T_{x, r_{i}} \phi \rightarrow v .
$$

Definition 2.7 (Lower and upper densities) Suppose $d$ is a fixed homogeneous left-invariant metric on $\mathbb{G}$. If $\phi$ is a Radon measure on $\mathbb{G}$, and $h>0$, we define

$$
\Theta_{d, *}^{h}(\phi, x):=\liminf _{r \rightarrow 0} \frac{\phi\left(B_{d}(x, r)\right)}{r^{h}}, \quad \text { and } \quad \Theta_{d}^{h, *}(\phi, x):=\limsup _{r \rightarrow 0} \frac{\phi\left(B_{d}(x, r)\right)}{r^{h}},
$$


and we say that $\Theta_{d, *}^{h}(\phi, x)$ and $\Theta_{d}^{h, *}(\phi, x)$ are the lower and upper $h$-density of $\phi$ at the point $x \in \mathbb{G}$, respectively. Furthermore, we say that measure $\phi$ has $h$-density if

$$
0<\Theta_{d, *}^{h}(\phi, x)=\Theta_{d}^{h, *}(\phi, x)<\infty, \quad \text { for } \phi \text {-almost any } x \in \mathbb{G} \text {. }
$$

When the metric $d$ is understood, we will tacitly drop the dependence on the metric in the notation.

Proposition 2.2 Assume $\phi$ is a Radon measure on $\mathbb{G}$ and suppose that $r_{i}^{-h} T_{x, r_{i}} \phi \rightarrow v$. Then, for any $z \in \operatorname{supp}(v)$ there exists a sequence $y_{i} \in \operatorname{supp}(\phi)$ such that $\delta_{1 / r_{i}}\left(x^{-1} y_{i}\right) \rightarrow z$.

Proof A simple argument by contradiction yields the claim. The proof follows verbatim its Euclidean analogue, see for instance the proof of [10, Proposition 3.4].

Definition 2.8 (Definition of $E(\vartheta, \gamma)$ ) Let $\phi$ be a Radon measure on $\mathbb{G}$ that is supported on the compact set $K$, i.e., such that $\phi(\mathbb{G} \backslash K)=0$. For any $\vartheta, \gamma \in \mathbb{N}$ we define

$$
E(\vartheta, \gamma):=\left\{x \in K: \vartheta^{-1} r^{h} \leq \phi(B(x, r)) \leq \vartheta r^{h} \text { for any } 0<r<1 / \gamma\right\} .
$$

The following two propositions can be found in [4, Proposition 2.4 and Proposition 2.5].

Proposition 2.3 ([4, Proposition 2.4 and Proposition 2.5]) Assume $\phi$ is a Radon measure supported on the compact set $K$ such that $0<\Theta_{*}^{h}(\phi, x) \leq \Theta^{h, *}(\phi, x)<\infty$ for $\phi$-almost every $x \in \mathbb{G}$. Then, for every $\vartheta, \gamma \in \mathbb{N}$ the set $E(\vartheta, \gamma)$ is compact and $\phi\left(\mathbb{G} \backslash \bigcup_{\vartheta, \gamma \in \mathbb{N}} E(\vartheta, \gamma)\right)=0$.

Proposition 2.4 Suppose $\phi$ is a Radon measure with h-density. Then for $\phi$-almost every $x \in \mathbb{G}$ we have that $\operatorname{Tan}_{h}(\phi, x)$ is not empty and for any $v \in \operatorname{Tan}_{h}(\phi, x)$ we have $0 \in \operatorname{supp}(v)$, and $\nu(B(y, s))=\Theta^{h}(\phi, x) s^{h}$ for any $y \in \operatorname{supp}(v)$ and any $s>0$.

Proof The proof follows verbatim its Euclidean counterpart, see for instance [10, Proposition 3.4].

Proposition 2.5 Suppose that $\mu$ is a Borel regular measure on $\mathbb{G}$ supported on a homogeneous subgroup $\mathbb{V} \in \mathbb{G}(h)$, such that $0 \in \operatorname{supp}(\mu)$ and assume that there exists a constant $C>0$ such that for any $z \in \operatorname{supp}(\mu)$ and any $s>0$ we have

$$
\mu(B(z, s))=C s^{h} .
$$

Then $\mu$ is a Haar measure of the subgroup $\mathbb{V}$.

Proof Without loss of generality we can assume $C=1$. Thanks to [18, Theorem 3.1] we thus infer that $\mu=\mathcal{C}^{h}\llcorner\operatorname{supp}(\mu)$. Moreover, for any $x \in \operatorname{supp}(\mu)$, thanks to Lemma 2.1, we have that $\mu(B(x, r))=\mathcal{C}^{h}\llcorner\mathbb{V}(B(x, r))$ for every $r>0$. If by contradiction $\operatorname{supp}(\mu) \neq \mathbb{V}$, then there would exist a $p \in \mathbb{V}$ and a $r_{0}>0$ such that $B\left(p, r_{0}\right) \cap \operatorname{supp}(\mu)=\emptyset$. This however is impossible since we would have

$$
\begin{aligned}
& \mathcal{C}^{h}\left\llcorner\mathbb{V}\left(B\left(0,2\left(\|p\|+r_{0}\right)\right)\right)\right. \\
& \quad \geq \mathcal{C}^{h}\left(B\left(0,2\left(\|p\|+r_{0}\right)\right) \cap \operatorname{supp}(\mu)\right)+\mathcal{C}^{h}\left(B\left(p, r_{0}\right) \cap \mathbb{V}\right)>\mu\left(B\left(0,2\left(\|p\|+r_{0}\right)\right)\right),
\end{aligned}
$$

and this is a contradiction with what we found above, since by assumption $0 \in \operatorname{supp}(\mu)$.

A very useful property of locally asymptotically doubling measures is that Lebesgue theorem holds and thus local properties are stable under restriction to Borel subsets. The forthcoming result is a direct consequence of [25, Theorem 3.4.3] and the Lebesgue differentiation Theorem in [25, page 77]. 
Proposition 2.6 Suppose $d$ is a fixed homogeneous left-invariant metric on $\mathbb{G}$ and that $\phi$ is a Radon measure on $\mathbb{G}$ such that for $\phi$-almost every $x \in \mathbb{G}$ we have

$$
\limsup _{r \rightarrow 0} \frac{\phi\left(B_{d}(x, 2 r)\right)}{\phi\left(B_{d}(x, r)\right)}<\infty \text {. }
$$

Then

(i) for any Borel set $B \subseteq \mathbb{G}$ the measure $\phi\llcorner B$ is a locally asymptotically doubling measure, and we have that the following equalities hold for $\phi$-almost every $x \in B$

$$
\Theta_{d, *}^{h}\left(\phi \llcorner B , x ) = \Theta _ { d , * } ^ { h } ( \phi , x ) , \quad \text { and } \quad \Theta _ { d } ^ { h , * } \left(\phi\llcorner B, x)=\Theta_{d}^{h, *}(\phi, x),\right.\right.
$$

(ii) for every non-negative $\rho \in L^{1}(\phi)$, and for $\phi$-almost every $x \in \mathbb{G}$ we have $\operatorname{Tan}(\rho \phi, x)=$ $\rho(x) \operatorname{Tan}(\phi, x)$. More precisely, for $\phi$-almost every $x \in \mathbb{G}$ the following holds

$$
\text { if } r_{i} \rightarrow 0 \text { is such that } c_{i} T_{x, r_{i}} \phi \rightarrow v \text { then } c_{i} T_{x, r_{i}}(\rho \phi) \rightarrow \rho(x) v .
$$

We recall that any Radon measure $\phi$ on $(\mathbb{G}, d)$ that satisfies (5) for $\phi$-almost every $x \in \mathbb{G}$ is said to be locally asymptotically doubling, or simply asymptotically doubling.

\subsection{Intrinsic Grassmannian in Carnot groups}

We recall in this section some useful properties about homogeneous subgroups in Carnot groups. We equip $\mathbb{G}$ with a fixed left-invariant homogeneous distance $d$ that will sometimes be understood.

Definition 2.9 (Intrinsic Grassmanian on Carnot groups) For any $1 \leq h \leq Q$, we define $\mathbb{G}(h)$ to be the family of homogeneous subgroups $\mathbb{V}$ of $\mathbb{G}$ that have Hausdorff dimension $h$. Let us recall that if $\mathbb{V}$ is a homogeneous subgroup of $\mathbb{G}$, any other homogeneous subgroup $\mathbb{L}$ such that

$$
\mathbb{V} \cdot \mathbb{L}=\mathbb{G}, \quad \text { and } \quad \mathbb{V} \cap \mathbb{L}=\{0\},
$$

is said to be a complement of $\mathbb{G}$. We let $\mathbb{G}_{c}(h)$ to be the subfamily of those $\mathbb{V} \in \mathbb{G}(h)$ that have a complement and we will refer to $\mathbb{G}_{c}(h)$ as the $h$-dimensional complemented Grassmanian. Finally, for any $1 \leq h \leq Q$ we endow $\mathbb{G}(h)$ with the metric

$$
d_{\mathbb{G}}\left(\mathbb{W}_{1}, \mathbb{W}_{2}\right):=d_{H, \mathbb{G}}\left(\mathbb{W}_{1} \cap B(0,1), \mathbb{W}_{2} \cap B(0,1)\right),
$$

where $d_{H, \mathbb{G}}$ is the Hausdorff distance of sets induced by the distance $d$. For more details we refer to [4].

We recall here the following proposition from [4, Proposition 2.7] that will be used several times.

Proposition 2.7 (Compactness of the Grassmannian) For any $1 \leq h \leq Q\left(\mathbb{G}(h), d_{\mathbb{G}}\right)$ is a compact metric space.

Definition 2.10 (Projections related to a splitting) For any $\mathbb{V} \in \mathbb{G}_{c}(h)$, if we choose a complement $\mathbb{L}$ of $\mathbb{V}$, we can find two unique elements $g_{\mathbb{V}}:=P_{\mathbb{V}} g \in \mathbb{V}$ and $g_{\mathbb{L}}:=P_{\mathbb{L}} g \in \mathbb{L}$ such that

$$
g=P_{\mathbb{V}}(g) \cdot P_{\mathbb{L}}(g)=g_{\mathbb{V}} \cdot g_{\mathbb{L}} .
$$

We will refer to $P_{\mathbb{V}}(g)$ and $P_{\mathbb{L}}(g)$ as the splitting projections, or simply projections, of $g$ onto $\mathbb{V}$ and $\mathbb{L}$, respectively. 
Proposition 2.8 ([17, Proposition 2.12 and Corollary 2.15], [4, Proposition 2.14]) Suppose d is a fixed homogeneous left-invariant metric on $\mathbb{G}$ and let $\|\cdot\|_{d}$ be the associated homogeneous norm. Then, for any $\mathbb{V} \in \mathbb{G}_{c}(h)$ with complement $\mathbb{L}$ there is a constant $C_{1}(d, \mathbb{V}, \mathbb{L})>0$ such that for any $p \in \mathbb{G}$ we have

$$
\begin{aligned}
& C_{1}(d, \mathbb{V}, \mathbb{L})\left\|P_{\mathbb{L}}(p)\right\|_{d} \leq \operatorname{dist}(p, \mathbb{V}) \leq\left\|P_{\mathbb{L}}(p)\right\|_{d}, \\
& C_{1}(d, \mathbb{V}, \mathbb{L})\left(\left\|P_{\mathbb{L}}(p)\right\|_{d}+\left\|P_{\mathbb{V}}(p)\right\|_{d}\right) \leq\|p\|_{d} \leq\left\|P_{\mathbb{L}}(p)\right\|_{d}+\left\|P_{\mathbb{V}}(p)\right\|_{d} .
\end{aligned}
$$

Furthermore, for any $r>0$, there exists a constant $C_{2}$ we have $\mathcal{S}_{d}^{h}\left\llcorner\mathbb{V}\left(P_{\mathbb{V}}\left(B_{d}(p, r)\right)\right)=\right.$ $C_{2}(d, \mathbb{V}, \mathbb{L}) r^{h}$ and, for any Borel set $A \subseteq \mathbb{G}$ for which $\mathcal{S}_{d}^{h}(A)<\infty$, we have

$$
\mathcal{S}_{d}^{h}\left\llcorner\mathbb{V}\left(P_{\mathbb{V}}(A)\right) \leq 2 C_{2}(d, \mathbb{V}, \mathbb{L}) \mathcal{S}_{d}^{h}(A) .\right.
$$

When the metric $d$ is understood, we will tacitly drop the dependence on the metric in the notation.

Remark 2.1 (About the definition of $C_{1}$ ) We stress that we fix $C_{1}$ to be the supremum of all the constants for which the inequalities in (7) hold.

We recall here the following proposition that will be useful later on.

Proposition 2.9 ([4, Proposition 2.10], [17, Proof of Lemma 2.20]) Suppose d is a fixed homogeneous left-invariant metric on $\mathbb{G}$. Let us fix $\mathbb{V} \in \mathbb{G}_{c}(h)$ and $\mathbb{L}$ two complementary homogeneous subgroups of $\mathbb{G}$. Then, for any $x \in \mathbb{G}$ the map $\Psi: \mathbb{V} \rightarrow \mathbb{V}$ defined as $\Psi(z):=P_{\mathbb{V}}(x z)$ is invertible and it has unitary Jacobian. As a consequence $\mathcal{S}_{d}^{h}\left(P_{\mathbb{V}}(\mathcal{E})\right)=$ $\mathcal{S}_{d}^{h}\left(P_{\mathbb{V}}\left(x P_{\mathbb{V}}(\mathcal{E})\right)\right)=\mathcal{S}_{d}^{h}\left(P_{\mathbb{V}}(x \mathcal{E})\right)$ for every $x \in \mathbb{G}$ and $\mathcal{E} \subseteq \mathbb{G}$ Borel.

The following proposition will be useful in the proof of Corollary 5.2. We omit the proof. The first part of the statement, i.e., the one about the homeomorphism, can be reached by using elementary Linear Algebra; while the second part of the statement follows from the first. We stress that in the following statement we are endowing $\mathfrak{g}$, that is identified with $\mathbb{R}^{n}$ through a choice of a basis of left-invariant vector fields $X_{1}, \ldots, X_{n}$, with a scalar product $\langle\cdot, \cdot\rangle$ that makes $X_{1}, \ldots, X_{n}$ orthonormal.

Proposition 2.10 Let $\mathcal{L}(\mathfrak{g}, \mathfrak{g})$ be the set of linear maps from the Lie algebra $\mathfrak{g}$ of $\mathbb{G}$ into itself, endowed with the operator norm $\rho$. Then, being $\mathbb{G}(\mathfrak{g})$ the Grassmannian of the vector space $\mathfrak{g}$, the map $\mathfrak{P}: \mathbb{G}(\mathfrak{g}) \rightarrow \mathcal{L}(\mathfrak{g}, \mathfrak{g})$ defined as $\mathfrak{P}(V):=\Pi_{V^{\perp}}$, where $\Pi_{V^{\perp}}$ is the orthogonal projection onto $V^{\perp}$, is a homeomorphism onto its image.

Then, a map $V: \mathfrak{g} \rightarrow \exp ^{-1}(\mathbb{G}(h))$ is Borel measurable if and only if the projection map $\pi_{V^{\perp}}: \mathfrak{g} \rightarrow \mathcal{L}(\mathfrak{g}, \mathfrak{g})$ defined as

$$
\pi_{V^{\perp}}(x):=\Pi_{V(x)^{\perp}},
$$

where $\Pi_{V(x)^{\perp}}$ is the orthogonal projection onto $V(x)^{\perp}$, is Borel measurable. In addition to this, the Borelianity of $\pi_{V^{\perp}}$ is also equivalent to saying that for any fixed $v, w \in \mathfrak{g}$, the map $x \mapsto\left\langle v, \Pi_{V(x)^{\perp}}[w]\right\rangle$ is Borel.

\subsection{Cones over homogeneous subgroups and intrinsic Lipschitz functions}

In this section we recall some basic definitions about intrinsic cones in Carnot groups. 
Definition 2.11 (Intrinsic cone) Suppose $d$ is an homogeneous left-invariant distance on $\mathbb{G}$. For any $\alpha>0$ and $\mathbb{V} \in \mathbb{G}(h)$, we define the cone $C_{\mathbb{V}, d}(\alpha)$ as:

$$
C_{\mathbb{V}, d}(\alpha):=\left\{w \in \mathbb{G}: \operatorname{dist}_{d}(w, \mathbb{V}) \leq \alpha\|w\|_{d}\right\} .
$$

Furthermore, given $\mathbb{V} \in \mathbb{G}(h)$ and an $\alpha>0$, we say that a Borel set $E \subseteq \mathbb{G}$ is a $C_{\mathbb{V}, d}(\alpha)$-set if

$$
E \subseteq p C_{\mathbb{V}, d}(\alpha) \quad \text { for any } p \in E .
$$

When the metric $d$ is understood, we will tacitly drop the dependence on the metric in the notation.

Remark 2.2 (Equivalent intrinsic cones) Suppose $d$ is a homogeneous left-invariant distance on $\mathbb{G}$. For some benefits toward the rest of the paper, let us prove that if $\mathbb{V} \in \mathbb{G}_{c}(h), \mathbb{L}$ is a complementary subgroup of $\mathbb{V}$, and $\alpha<C_{1}(d, \mathbb{V}, \mathbb{L})$, then

$$
C_{\mathbb{V}, d}(\alpha) \subseteq\left\{w \in \mathbb{G}:\left\|P_{\mathbb{L}} w\right\|_{d} \leq \frac{\alpha}{C_{1}(d, \mathbb{V}, \mathbb{L})-\alpha}\left\|P_{\mathbb{V}} w\right\|_{d}\right\} .
$$

Indeed, let us take an element $w$ in the complement of the set in the right-hand-side above. Thanks to the fact that $\|w\|_{d} \leq\left\|P_{\mathbb{L}} w\right\|_{d}+\left\|P_{\mathbb{V}} w\right\|_{d}<C_{1}(d, \mathbb{V}, \mathbb{L}) \alpha^{-1}\left\|P_{\mathbb{L}} w\right\|_{d}$ and to Corollary 2.8 we have

$$
\operatorname{dist}_{d}(w, \mathbb{V}) \geq C_{1}(d, \mathbb{V}, \mathbb{L})\left\|P_{\mathbb{L}}(w)\right\|_{d}>\alpha\|w\|_{d} .
$$

Therefore, any such $w$ is contained in the complement of the left-hand-side of (9), and thus we get the sought conclusion. Moreover, for any $\mathbb{V} \in \mathbb{G}_{c}(h)$ and any of its complementary subgroup $\mathbb{L}$, let us show that for any $\alpha>0$

$$
C_{\mathbb{V}, \mathbb{L}, d}(\alpha):=\left\{w \in \mathbb{G}:\left\|P_{\mathbb{L}} w\right\|_{d} \leq \alpha\left\|P_{\mathbb{V}} w\right\|_{d}\right\} \subseteq C_{\mathbb{V}, d}\left(C_{1}(d, \mathbb{V}, \mathbb{L})^{-1} \alpha\right) .
$$

Indeed, if $w$ is an element in the left-hand-side above, we can readily see thanks to Corollary 2.8 that

$$
\operatorname{dist}_{d}(w, \mathbb{V}) \leq\left\|P_{\mathbb{L}} w\right\|_{d} \leq \alpha\left\|P_{\mathbb{V}} w\right\|_{d} \leq \alpha C_{1}(d, \mathbb{V}, \mathbb{L})^{-1}\|w\|_{d} .
$$

All in all we have proved that if $\mathbb{V} \in \mathbb{G}_{c}(h), \mathbb{L}$ is one of its complementary subgroups and $\alpha<C_{1}(d, \mathbb{V}, \mathbb{L})$ we have

$$
C_{\mathbb{V}, \mathbb{L}, d}\left(C_{1}(d, \mathbb{V}, \mathbb{L}) \alpha\right) \subseteq C_{\mathbb{V}, d}(\alpha) \subseteq C_{\mathbb{V}, \mathbb{L}, d}\left(\alpha /\left(C_{1}(d, \mathbb{V}, \mathbb{L})-\alpha\right)\right),
$$

thus showing that, below some threshold on the opening, the cones $C_{\mathbb{V}, d}$ and $C_{\mathbb{V}, \mathbb{L}, d}$ are equivalent.

Remark 2.3 (Equivalent distances and cones) Let $d_{1}, d_{2}$ be two homogeneous and leftinvariant metrics on $\mathbb{G}$. Since they are bi-Lipschitz equivalent, we can find a constant $0<\mathfrak{c}<1$ such that $\mathfrak{c} d_{1}(x, y) \leq d_{2}(x, y) \leq \mathfrak{c}^{-1} d_{1}(x, y)$ for any $x, y \in \mathbb{G}$. Then for any $\alpha>0$ and any $\mathbb{V} \in \mathbb{G}(h)$ we have

$$
C_{\mathbb{V}, d_{1}}\left(\mathfrak{c}^{2} \alpha\right) \subseteq C_{\mathbb{V}, d_{2}}(\alpha) \subseteq C_{\mathbb{V}, d_{1}}\left(\mathfrak{c}^{-2} \alpha\right) .
$$

We now recall two results that were already proven in [4]. We refer the reader to the reference [4] for the simple proofs. 
Lemma 2.11 ([4, Lemma 2.16]) Suppose d is a homogeneous left-invariant distance on $\mathbb{G}$. For any $\mathbb{V} \in \mathbb{G}_{c}(h)$, given $\mathbb{L}$ to be a complementary subgroup of $\mathbb{V}$, there exists $0<\varepsilon_{1}:=$ $\varepsilon_{1}(d, \mathbb{V}, \mathbb{L})<1$ such that

$$
\mathbb{L} \cap C_{\mathbb{V}, d}\left(\varepsilon_{1}\right)=\{0\} .
$$

and for the aims of this paper, we can and will fix $\varepsilon_{1}(d, \mathbb{V}, \mathbb{L}):=C_{1}(d, \mathbb{V}, \mathbb{L}) / 2$.

Proposition 2.12 Suppose $d$ is a homogeneous left-invariant distance on $\mathbb{G}$. The function $\mathfrak{e}: \mathbb{G}_{c}(h) \rightarrow \mathbb{R}$ defined as

$$
\mathfrak{e}(\mathbb{V}):=\sup \left\{\varepsilon_{1}(d, \mathbb{V}, \mathbb{L}): \mathbb{L} \text { is a complement of } \mathbb{V}\right\},
$$

is lower semicontinuous. In particular the following conclusion holds

- if $\mathscr{G} \subseteq \mathbb{G}_{c}(h)$ is compact with respect to $d_{\mathbb{G}}$, then there exists a eqG $>0$ such that $\mathfrak{e}(\mathbb{V}) \geq \mathfrak{e} \mathscr{G}$ for any $\mathbb{V} \in \mathscr{G}$.

Proof It follows verbatim from the proof of [4, Proposition 2.22], taking [4, Remark 2.5] into account.

Let us recall the classical definition of intrinsic Lipschits function, see [17, Definition 11].

Definition 2.12 (Intrinsic Lipschitz function) Let $\mathbb{W} \in \mathbb{G}_{c}(h)$, assume $\mathbb{L}$ is a complement of $\mathbb{W}$, and let $E \subseteq \mathbb{W}$. A function $f: E \rightarrow \mathbb{L}$ is said to be an intrinsic Lipschitz function if there exists an $\alpha>0$ such that for every $p \in \operatorname{graph}(f):=\{v \cdot f(v): v \in E\}$ we have

$$
\operatorname{graph}(f) \cap p C_{\mathbb{W}, \mathbb{L}}(\alpha)=\operatorname{graph}(f),
$$

where the cones $C_{\mathbb{W}, \mathbb{L}}(\alpha)$ have been defined in Remark 2.2.

We finally state two properties of intrinsic Lipschitz graphs that will be useful later on, and whose simple proofs can be found in [4].

Proposition 2.13 ([4, Proposition 2.19]) Suppose d is a homogeneous left-invariant distance on $\mathbb{G}$ and let us fix $\mathbb{V} \in \mathbb{G}_{c}(h)$ with complement $\mathbb{L}$. If $\Gamma \subset \mathbb{G}$ is a $C_{\mathbb{V}}(\alpha)$-set for some $\alpha \leq \varepsilon_{1}(d, \mathbb{V}, \mathbb{L})$, then the map $P_{\mathbb{V}}: \Gamma \rightarrow \mathbb{V}$ is injective. As a consequence $\Gamma$ is the intrinsic graph of an intrinsically Lipschitz map defined on $P_{\mathbb{V}}(\Gamma)$.

Lemma 2.14 ([4, Lemma 2.21]) Let $\mathbb{L}$ and $\mathbb{V}$ be homogeneous complementary subgroups of $\mathbb{G}$ endowed with a left-invariant homogeneous distance $d$. Suppose $\Gamma$ is a $C_{\mathbb{V}, d}(\alpha)$-set with $\alpha \leq \varepsilon_{1}(d, \mathbb{V}, \mathbb{L})$. Then there exists a constant $\mathfrak{C}(\alpha)=\mathfrak{C}(\alpha, d, \mathbb{V}, \mathbb{L})>0$ such that

$$
\mathcal{S}_{d}^{h}\left(P_{\mathbb{V}}\left(B_{d}(x, r) \cap \Gamma\right)\right) \geq \mathcal{S}_{d}^{h}\left(P_{\mathbb{V}}\left(B_{d}(x, \mathfrak{C}(\alpha) r) \cap x C_{\mathbb{V}, d}(\alpha)\right) \cap P_{\mathbb{V}}(\Gamma)\right),
$$

for any $x \in \Gamma$, and any $r>0$.

\subsection{Rectifiable measures in Carnot groups}

In what follows we are going to define the class of $h$-flat measures on a Carnot group and then we will give proper definitions of rectifiable measures on Carnot groups. 
Definition 2.13 (Flat measures) For any $h \in\{1, \ldots, Q\}$ we let $\mathfrak{M}(h)$ to be the family of flat $h$-dimensional measures in $\mathbb{G}$, i.e.,

$$
\mathfrak{M}(h):=\left\{\lambda \mathcal{S}^{h}\llcorner\mathbb{W}: \text { for some } \lambda>0 \text { and } \mathbb{W} \in \mathbb{G}(h)\} .\right.
$$

Furthermore, if $G$ is a subset of the $h$-dimensional Grassmanian $\mathbb{G}(h)$, we let $\mathfrak{M}(h, G)$ to be the set

$$
\mathfrak{M}(h, G):=\left\{\lambda \mathcal{S}^{h}\llcorner\mathbb{W}: \text { for some } \lambda>0 \text { and } \mathbb{W} \in G\}\right.
$$

Definition $2.14\left(\mathscr{P}_{h}\right.$ and $\mathscr{P}_{h}^{c}$-rectifiable measures) Let $h \in\{1, \ldots, Q\}$. A Radon measure $\phi$ on $\mathbb{G}$ is said to be a $\mathscr{P}_{h}$-rectifiable measure if for $\phi$-almost every $x \in \mathbb{G}$ we have

(i) $0<\Theta_{*}^{h}(\phi, x) \leq \Theta^{h, *}(\phi, x)<+\infty$,

(ii) there exists a $\mathbb{V}(x) \in \mathbb{G}(h)$ such that $\operatorname{Tan}_{h}(\phi, x) \subseteq\left\{\lambda \mathcal{S}^{h}\llcorner\mathbb{V}(x): \lambda \geq 0\}\right.$.

Furthermore, we say that $\phi$ is $\mathscr{P}_{h}^{c}$-rectifiable if (ii) is replaced with the weaker

(ii)* there exists a $\mathbb{V}(x) \in \mathbb{G}_{c}(h)$ such that $\operatorname{Tan}_{h}(\phi, x) \subseteq\left\{\lambda \mathcal{S}^{h}\llcorner\mathbb{V}(x): \lambda \geq 0\}\right.$.

Remark 2.4 (About $\lambda=0$ in Definition 2.14) It is readily noticed that, since in Definition 2.14 we are asking $\Theta_{*}^{h}(\phi, x)>0$ for $\phi$-almost every $x$, we can not have the zero measure as an element of $\operatorname{Tan}_{h}(\phi, x)$ thanks to [4, Proposition 2.26]. As a consequence, a posteriori, we have that in item (ii) and item (ii)* we can restrict to $\lambda>0$. We will tacitly work in this restriction from now on, see [4, Remark 2.7].

Remark 2.5 (About the rectifiability of Hausdorff measures) We observe that if $\Gamma$ is a Borel set in $\mathbb{G}, \mathcal{S}^{h}\left\llcorner\Gamma\right.$ is $\mathscr{P}_{h}$-rectifiable if and only if $\mathcal{C}^{h}\left\llcorner\Gamma\right.$ (or also $\mathcal{H}^{h}\left\llcorner\Gamma\right.$ ) is $\mathscr{P}_{h}$-rectifiable. This is because $\mathcal{S}^{h}, \mathcal{H}^{h}, \mathcal{C}^{h}$ are equivalent measures (see Definition 2.4), the $\mathscr{P}_{h}$-rectifiability implies being locally asymptotically doubling, and then we can transfer the property of being $\mathscr{P}_{h}$ rectifiable from one measure to the other by using Lebesgue-Radon-Nikodym theorem (see [25, page 82]) and the locality of tangents in Proposition 2.6.

We introduce now a way to estimate how far two measures are.

Definition 2.15 (Definition of $F_{K}$ ) Given $\phi$ and $\psi$ two Radon measures on $\mathbb{G}$, and given $K \subseteq \mathbb{G}$ a compact set, we define

$$
F_{K}(\phi, \psi):=\sup \left\{\left|\int f d \phi-\int f d \psi\right|: f \in \operatorname{Lip}_{1}^{+}(K)\right\},
$$

where $\operatorname{Lip}_{1}^{+}(K)$ denotes the class of 1-Lipschitz nonnegative function with compact support contained in $K$. We also write $F_{x, r}$ for $F_{B(x, r)}$.

Remark 2.6 (Properties of $F_{K}$ ) With few computations that we omit, it is easy to see that $F_{x, r}(\phi, \psi)=r F_{0,1}\left(T_{x, r} \phi, T_{x, r} \psi\right)$. Furthermore, $F_{K}$ enjoys the triangular inequality. Indeed, if $\phi_{1}, \phi_{2}, \phi_{3}$ are Radon measures and $f \in \operatorname{Lip}_{1}^{+}(K)$, then

$$
\begin{aligned}
& \left|\int f d \phi_{1}-\int f d \phi_{2}\right| \leq \mid \int f d \phi_{1} \\
& \quad-\int f d \phi_{3}|+| \int f d \phi_{3}-\int f d \phi_{2} \mid \leq F_{K}\left(\phi_{1}, \phi_{2}\right)+F_{K}\left(\phi_{2}, \phi_{3}\right) .
\end{aligned}
$$

The arbitrariness of $f$ concludes that $F_{K}\left(\phi_{1}, \phi_{2}\right) \leq F_{K}\left(\phi_{1}, \phi_{3}\right)+F_{K}\left(\phi_{3}, \phi_{2}\right)$. 
Definition 2.16 (Definition of $F_{r}$ ) For a given Radon measure $\phi$ on $\mathbb{G}$ and for $r>0$, let us define $F_{r}(\phi):=\int \operatorname{dist}\left(z, U(0, r)^{c}\right) d \phi(z)$.

Lemma 2.15 For any Radon measure $\phi$ on $\mathbb{G}$ and any $r>0$ we have that $F_{r}(\phi)=F_{0, r}(\phi, 0)$.

Proof It is immediate to see that $F_{0, r}(\phi, 0) \geq F_{r}(\phi)$ for any $r>0$. In order to prove the viceversa, note that for any $f \in \operatorname{Lip}_{1}^{+}(B(0, r))$ we have that $\left.f\right|_{\partial B(0, r)}=0$. Thanks to this observation, for any $y \in B(0, r)$ if we let $x \in \partial B(0, r)$ be a point of minimal distance of $y$ from $U(0, r)^{c}$ we have

$$
f(y)=|f(y)-f(x)| \leq d(y, x)=\operatorname{dist}\left(y, U(0, r)^{c}\right),
$$

and this finally shows that $F_{0, r}(\phi, 0)=F_{r}(\phi)$, concluding the proof of the lemma.

Proposition 2.16 The function defined on $\mathcal{M} \times \mathcal{M}$ as

$$
d(\phi, \psi):=\sum_{p=0}^{\infty} 2^{-p} \min \left\{1, F_{0, p}(\phi, \psi)\right\},
$$

is a distance and $(\mathcal{M}, d)$ is a separable metric space. The topology induced by $d$ on $\mathcal{M}$ coincides with the one given by the weak* topology.

Moreover, let us assume $\left\{\phi_{i}\right\}_{i \in \mathbb{N}}$ is a sequence of Radon measures such that $\lim _{\sup } \operatorname{su}_{i \rightarrow \infty} \phi_{i}$ $(B(0, r))<\infty$ for every $r>0$. Then $\left\{\phi_{i}\right\}_{i \in \mathbb{N}}$ has a converging subsequence with respect to the weak * topology.

Proof The result is stated in [42, Proposition 1.12] in the Euclidean case, but the proof works verbatim for Radon measures on Carnot groups.

Proposition 2.17 The function $F_{0,1}(\cdot, \cdot)$ is a metric on $\mathfrak{B}(h):=\left\{\psi \in \mathfrak{M}(h): F_{1}(\psi)=1\right\}$ and $\left(\mathfrak{B}(h), F_{0,1}\right)$ is a compact metric space.

Proof First of all, we note that for any $\mu, v \in \mathfrak{B}(h)$ we have that $F_{0,1}(\mu, v)=0$ if and only if $\mu=v$ and this is an immediate consequence of the fact that $\mu$ and $v$ are cones. Symmetry follows directly form the definition and the triangular inequality follows from Remark 2.6.

We are left to show that $\left(\mathfrak{B}(h), F_{0,1}\right)$ is a compact metric space. Let $\Psi_{i}$ be a sequence in $\mathfrak{B}(h)$ and note that since $\mathcal{C}^{h}\llcorner\mathbb{V}(B(0,1))=1$ for every $\mathbb{V} \in \mathbb{G}(h)$, because of Lemma 2.1 , we deduce that $\Psi_{i}=(h+1) \mathcal{C}^{h}\left\llcorner\mathbb{V}_{i}\right.$ for some $\mathbb{V}_{i} \in \mathbb{G}(h)$. Thus, we can find a (nonrelabeled) subsequence of the planes $\mathbb{V}_{i}$ that converges to some $\mathbb{V} \in \mathbb{G}(h)$ in the Hausdorff metric thanks to the compactness of the Grassmanian $\mathbb{G}(h)$, see Proposition 2.7. Hence, by [4, Proposition 2.29] we infer that $\Psi_{i} \rightarrow(h+1) \mathcal{C}^{h}\llcorner\mathbb{V} \in \mathfrak{B}(h)$ and therefore the compactness follows.

Now we are going to define some functionals that quantifies how far is a measure from being flat around a point $x \in \mathbb{G}$ and at a certain scale $r>0$.

Definition 2.17 (Definition of $d_{x, r}$ ) For any $x \in \mathbb{G}$, any $h \in\{1, \ldots, Q\}$ and any $r>0$ we define the functional

$$
d_{x, r}(\phi, \mathfrak{M}(h)):=\inf _{\mathbb{V} \in \mathbb{G}(h)} F_{0,1}\left(T_{x, r} \phi / F_{1}\left(T_{x, r} \phi\right),(h+1) \mathcal{C}^{h}\llcorner\mathbb{V}) .\right.
$$

Furthermore, if $G$ is a subset of the $h$-dimensional Grassmanian $\mathbb{G}(h)$, we also define

$$
d_{x, r}(\phi, \mathfrak{M}(h, G)):=\inf _{\mathbb{V} \in G} F_{0,1}\left(T_{x, r} \phi / F_{1}\left(T_{x, r} \phi\right),(h+1) \mathcal{C}^{h}\llcorner\mathbb{V}) .\right.
$$


Remark 2.7 (About the definition of $d_{x, r}$ ) For any Radon measure $\phi$ on $\mathbb{G}$ and any $r>0$ it is immediate to see that $F_{1}\left(T_{0, r} \phi\right)=r^{-1} F_{r}(\phi)$. Moreover, thanks to the first part of Remark 2.6, we get by few simple computations

$$
F_{0,1}\left(\frac{T_{x, r} \phi}{F_{1}\left(T_{x, r} \phi\right)},(h+1) \mathcal{C}^{h}\llcorner\mathbb{V})=r^{-(h+1)} F_{0, r}\left(\frac{T_{x, 1} \phi}{r^{-(h+1)} F_{r}\left(T_{x, 1} \phi\right)},(h+1) \mathcal{C}^{h}\llcorner\mathbb{V}),\right.\right.
$$

for all $r>0$ and $\mathbb{V} \in \mathbb{G}(h)$. Hence, since $F_{1}\left((h+1) \mathcal{C}^{h}\llcorner\mathbb{V})=1\right.$ as a consequence of [4, Proposition 2.12] and Lemma 2.1, we notice that the definition in (15) agrees with the definition given in $[42, \S 2.1(3)]$. Namely, $d_{x, r}(\phi, \mathfrak{M}(h, G))=d_{r}\left(T_{x, 1} \phi, \mathfrak{M}(h, G)\right)=$ $d_{1}\left(T_{x, r} \phi, \mathfrak{M}(h, G)\right)$, where $d_{r}$ is the one defined in [42, §2.1(3)].

For the sake of completeness, and for some benefits toward subsequent calculations, let us recall here the precise definition of the function $d$ Preiss gave in his setting. Let $\mathscr{C}$ be an arbitrary cone of measures without the origin, that means $0 \notin \mathscr{C}$ and $\mu \in \mathscr{C}$ implies $\lambda T_{0, v} \mu \in \mathscr{C}$ for every $\lambda, v>0$. Then, for every $r>0$ and $\phi$ a Radon measure we define

$$
d_{r}(\phi, \mathscr{C}):=\inf \left\{F_{r}\left(\frac{\phi}{F_{r}(\phi)}, \psi\right): \psi \in \mathscr{C}, F_{r}(\psi)=1\right\} .
$$

By the explicit expression and the continuity of $F_{r}(\cdot)$ with respect to the weak* convergence, one easily verifies that, for every $r>0$

$$
\phi_{k} \rightarrow_{k} \phi, F_{r}(\phi)>0 \Rightarrow d_{r}\left(\phi_{k}, \mathscr{C}\right) \rightarrow_{k} d_{r}(\phi, \mathscr{C}),
$$

compare [42, 2.1(6)]. Moreover, due to a slight modification of (16), we have, for every $r>0$ and every Radon measure $\phi$,

$$
d_{r}(\phi, \mathscr{C})=d_{1}\left(T_{0, r} \phi, \mathscr{C}\right) .
$$

We now adapt some classical results contained in [42] to our context. The aim will be to prove that when a Radon measure on $\mathbb{G}$ has a tangent at a point that is a cone (of measures) with compact basis, then the measure is locally asymptotically doubling. The following proposition is the analogue of [42, Propostion 2.2].

Proposition 2.18 Assume that $\mathcal{T}$ is a non-empty cone of Radon measures, i.e., for any $v \in \mathcal{T}$ and any $\lambda, \eta>0$ we have $\eta T_{0, \lambda} \nu \in \mathcal{T}$, and moreover $0 \notin \mathcal{T}$. Then, the following are equivalent

(i) the set $\mathfrak{B}(\mathcal{T}):=\left\{v \in \mathcal{T}: F_{1}(v)=1\right\}$ is weak* compact,

(ii) for any sequence $\left\{v_{i}\right\}_{i \in \mathbb{N}} \subseteq \mathcal{T}$ such that $\lim _{i \rightarrow \infty} F_{1}\left(v_{i}\right)=0$, we have $v_{i} \rightarrow 0$,

(iii) there is a $q \in(0, \infty)$ such that $v(B(0,2 r)) \leq q v(B(0, r))$ for every $r>0$ and any $v \in \mathcal{T}$.

Proof Let us first prove that (i) $\Rightarrow$ (ii). Let $v_{i}$ be a sequence in $\mathcal{T}$ and let us assume that $\lim _{i \rightarrow \infty} F_{1}\left(v_{i}\right)=0$. We note that $v_{i} \rightarrow 0$ if and only if $F_{0, t}\left(v_{i}, 0\right)=F_{t}\left(v_{i}\right) \rightarrow_{i} 0$ for any $t>0$. This means that if $v_{i}$ does not converge to 0 , we infer that there are a $t>0$ and an $\varepsilon>0$ such that, up to passing to subsequences, we have $F_{t}\left(v_{i}\right)>\varepsilon$ for any $i \in \mathbb{N}$. Let us define

$$
r_{i}:=\sup \left\{r \in[1, t]: F_{r}\left(v_{i}\right) \leq F_{1}\left(v_{i}\right)+1 / i\right\} .
$$

It is immediate to see that up to further subsequences $F_{1}\left(T_{0, r_{i}} v_{i}\right)=r_{i}^{-1} F_{r_{i}}\left(v_{i}\right)>0$ and that

$$
\lim _{i \rightarrow \infty} \frac{F_{t / r_{i}}\left(T_{0, r_{i}} v_{i}\right)}{F_{1}\left(T_{0, r_{i}} v_{i}\right)}=\lim _{i \rightarrow \infty} \frac{F_{t}\left(v_{i}\right)}{F_{r_{i}}\left(v_{i}\right)}>\varepsilon \lim _{i \rightarrow \infty}\left(F_{1}\left(v_{i}\right)+1 / i\right)^{-1}=\infty .
$$


Thanks to the fact that $\mathcal{T}$ is a cone, we know that $F_{1}\left(T_{0, r_{i}} v_{i}\right)^{-1} T_{0, r_{i}} v_{i} \in \mathfrak{B}(\mathcal{T})$ and thus there must exists a converging (non-relabeled) subsequence of $r_{i}$ and a $v \in \mathfrak{B}(\mathcal{T})$ such that $F_{1}\left(T_{0, r_{i}} v_{i}\right)^{-1} T_{0, r_{i}} v_{i} \rightarrow v$. This however implies that

$$
\infty=\lim _{i \rightarrow \infty} \frac{F_{t / r_{i}}\left(T_{0, r_{i}} v_{i}\right)}{F_{1}\left(T_{0, r_{i}} v_{i}\right)} \leq \lim _{i \rightarrow \infty} \frac{F_{t}\left(T_{0, r_{i}} v_{i}\right)}{F_{1}\left(T_{0, r_{i}} v_{i}\right)}=\lim _{i \rightarrow \infty} F_{t}\left(F_{1}\left(T_{0, r_{i}} v_{i}\right)^{-1} T_{0, r_{i}} v_{i}\right)=F_{t}(v),
$$

that is a contradiction with the fact that $v$ is a Radon measure.

Secondly, let us show that (ii) $\Rightarrow$ (iii). Since $\mathcal{T}$ is a cone, it suffices to prove that there exists $q \in(0,+\infty)$ such that $v(B(0,2)) \leq q v(B(0,1))$ for every $v \in \mathcal{T}$. Indeed, we thus would get that for every $v \in \mathcal{T}$ and $r>0$ we have $v(B(0,2 r))=T_{0, r} v(B(0,2)) \leq$ $q T_{0, r} v(B(0,1))=q v(B(0, r))$. Suppose by contradiction that there exists a sequence of measures $v_{i} \in \mathcal{T}$ such that $v_{i}(B(0,2))>i v_{i}(B(0,1))$. Note now that since $\mathcal{T}$ is a cone, the measures $v_{i}(B(0,2))^{-1} v_{i}$ are still in $\mathcal{T}$ and $\lim _{i \rightarrow \infty} F_{1}\left(v_{i}(B(0,2))^{-1} v_{i}\right)=0$. Thanks to (ii) this shows in particular that

$$
v_{i}(B(0,2))^{-1} v_{i} \rightarrow 0
$$

However, since $F_{3}\left(v_{i}(B(0,2))^{-1} v_{i}\right) \geq 1$ for any $i \in \mathbb{N}$, this is a contradiction with (20), according to which one should have

$$
\lim _{i \rightarrow \infty} F_{3}\left(v_{i}(B(0,2))^{-1} v_{i}\right)=F_{3}(0)=0,
$$

as clearly $F_{3}$ is a weak* continuous operator on Radon measures.

Finally, let us prove the implication (iii) $\Rightarrow$ (i). Let $\left\{v_{i}\right\}_{i \in \mathbb{N}}$ be a sequence in $\mathfrak{B}(\mathcal{T})$ and note that for any $i \in \mathbb{N}$ we have

$$
\nu_{i}(B(0,1 / 2)) \leq 2 F_{1}\left(v_{i}\right)=2,
$$

and thus thanks to (iii) we infer that for any $r>0$ we have $v_{i}(B(0, r)) \leq 4 \max \left\{1, q^{\log _{2}(r)+1}\right\}$ for any $i \in \mathbb{N}$. Proposition 2.16 and the weak* continuity of $F_{1}$ conclude the proof.

Remark 2.8 For some benefit towards the remaining part of this section, let us notice that if $\mathcal{T}$ is a non-empty cone of Radon measure such that $\mathfrak{B}(\mathcal{T})$ is weak* compact, for every $\lambda>1$ there is $\tau>1$ such that $F_{\tau r}(\psi) \leq \lambda F_{r}(\psi)$ for every $r>0$ and $\psi \in \mathcal{T}$. The proof follows verbatim from the five lines in $[42,(1) \Rightarrow(5)$ of Proposition 2.2].

Proposition 2.19 For any Radon measure $\phi$ on $\mathbb{G}$ and $\phi$-almost every $x \in \mathbb{G}$ the $\operatorname{set} \operatorname{Tan}(\phi, x)$ is either empty or a cone. Suppose $\phi$ is a Radon measure on $\mathbb{G}$ such that the set $\mathfrak{B}(\phi, x):=$ $\left\{v \in \operatorname{Tan}(\phi, x): F_{1}(v)=1\right\}$ is a non-empty weak* compact for $\phi$-almost every $x \in \mathbb{G}$. Then $\phi$ is locally asymptotically doubling.

Proof In order to prove the first part of the statement, let $x \in \operatorname{supp}(\phi)$ be a point where $\operatorname{Tan}(\phi, x)$ is non-empty, choose a $v \in \operatorname{Tan}(\phi, x)$ and assume that $r_{i}$ and $c_{i}$ are two sequences such that

$$
c_{i} T_{x, r_{i}} \phi \rightarrow v .
$$

To conclude the proof of the claim we need to show that for any $\eta, \lambda>0$ we have $\eta T_{0, \lambda} \nu \in$ $\operatorname{Tan}(\phi, x)$ and to do this, we just note that

$$
\eta c_{i} T_{x, \lambda r_{i}} \phi=\eta T_{0, \lambda}\left(c_{i} T_{x, r_{i}} \phi\right) \rightarrow \eta T_{0, \lambda} \nu .
$$

This shows that $\eta T_{0, \lambda} \nu \in \operatorname{Tan}(\phi, x)$ and thus $\operatorname{Tan}(\phi, x)$ is a cone. 
Fix a point in $\mathbb{G}$ where the set $\mathfrak{B}(\phi, x)$ is a compact cone and thanks to Proposition 2.18(iii) we infer there exists a $q>0$ such that $v(B(0,2 r)) \leq q v(B(0, r))$ for any $v \in \operatorname{Tan}(\phi, x)$ and every $r>0$. Let $\mathfrak{d}:=\inf \left\{\operatorname{dist}\left(z, U(0,1 / 2)^{c}\right): z \in B(0,1 / 4)\right\}>0$. We now prove that

$$
\limsup _{r \rightarrow 0} F_{1}\left(T_{x, 2 r} \phi\right) / F_{1}\left(T_{x, r} \phi\right) \leq 2 \mathfrak{d}^{-1} q^{2} \text {. }
$$

Indeed, if by contradiction $r_{i}$ is an infinitesimal sequence such that $F_{1}\left(T_{x, 2 r_{i}} \phi\right)>$ $2 \mathfrak{d}^{-1} q^{2} F_{1}\left(T_{x, r_{i}} \phi\right)$, then for any $v \in \mathfrak{B}(\phi, x)$ we have

$$
\begin{aligned}
& F_{1}\left(T_{x, 2 r_{i}} \phi / F_{1}\left(T_{x, 2 r_{i}} \phi\right), v\right) \geq F_{1 / 2}\left(T_{x, 2 r_{i}} \phi / F_{1}\left(T_{x, 2 r_{i}} \phi\right), v\right) \\
& \quad \geq F_{1 / 2}(v)-F_{1 / 2}\left(T_{x, 2 r_{i}} \phi\right) / F_{1}\left(T_{x, 2 r_{i}} \phi\right),
\end{aligned}
$$

where the last inequality comes from Remark 2.6 and Lemma 2.15. Furthermore, we also have for any $v \in \mathfrak{B}(\phi, x)$ that

$$
F_{1 / 2}(v)=\frac{F_{1 / 2}(v)}{F_{1}(v)} \geq \frac{\mathfrak{d} v(B(0,1 / 4))}{2 v(B(0,1))} \geq \frac{\mathfrak{d}}{2 q^{2}} .
$$

Thanks to the absurd hypothesis and the fact that for any $s>0$ we have $F_{s}\left(T_{x, r} \phi\right)=$ $s F_{1}\left(T_{x, r s} \phi\right)$, we infer that

$$
F_{1 / 2}\left(T_{x, 2 r_{i}} \phi\right) / F_{1}\left(T_{x, 2 r_{i}} \phi\right)=F_{1}\left(T_{x, r_{i}} \phi\right) / 2 F_{1}\left(T_{x, 2 r_{i}} \phi\right) \leq \mathfrak{d} / 4 q^{2} .
$$

Putting (22), (23) and (24) together, we conclude that

$$
F_{1}\left(T_{x, 2 r_{i}} \phi / F_{1}\left(T_{x, 2 r_{i}} \phi\right), v\right) \geq \mathfrak{d} / 4 q^{2} \geq \min \left\{\mathfrak{d} / 4 q^{2}, 1 / 2\right\}=: \varepsilon,
$$

for any $v \in \mathfrak{B}(\phi, x)$. Let us now denote, for simplicity, $\mathscr{T}:=\operatorname{Tan}(\phi, x)$. By taking into account the definition of $d_{1}$ in (17), we get from the previous computations that $d_{1}\left(T_{x, 2 r_{i}} \phi, \mathscr{T}\right) \geq \varepsilon$ for every $i$. Let us fix $v \in \operatorname{Tan}(\phi, x)$ such that $c_{i} T_{x, s_{i}} \phi \rightarrow v$ and let us note that (17) and (18) imply that

$$
\lim _{i \rightarrow 0} d_{1}\left(T_{x, s_{i}} \phi, \mathscr{T}\right)=\lim _{i \rightarrow \infty} d_{1}\left(c_{i} T_{x, s_{i}} \phi, \mathscr{T}\right)=d_{1}(\nu, \mathscr{T})=0 .
$$

Thanks to the above chain of identities, for $i$ sufficiently large, we denote by $\ell_{i}$ the smallest number among those $\ell \in\left[0, s_{i}\right]$ with the property that $d_{1}\left(T_{x, \eta}, \mathscr{T}\right)<\varepsilon$ for every $\ell<$ $\eta \leq s_{i}$. Since $d_{1}\left(T_{x, 2 r_{i}} \phi, \mathscr{T}\right) \geq \varepsilon$ we conclude that $\ell_{i}>0$ for $i$ sufficiently large and $d_{1}\left(T_{x, \ell_{i}} \phi, \mathscr{T}\right)=\varepsilon$ by the minimality of $\ell_{i}$ and the continuity of the map $\eta \mapsto d_{1}\left(T_{x, \eta} \phi, \mathscr{T}\right)$.

If, up to subsequences, $\ell_{i} / s_{i} \rightarrow_{i} t>0$, we conclude that, thanks to (18),

$$
\begin{aligned}
& d_{1}\left(T_{0, t} \nu, \mathscr{T}\right)=\lim _{i \rightarrow+\infty} d_{1}\left(T_{x, t s_{i}} \phi, \mathscr{T}\right) \\
& \quad \geq \varepsilon,
\end{aligned}
$$

where the last inequality is true since $t s_{i}$ is arbitrarily near to $\ell_{i}$ for $i$ large enough, and $d_{1}\left(T_{x, \ell_{i}} \phi, \mathscr{T}\right) \geq \varepsilon$. The previous inequality gives a contradiction since $T_{0, t} v \in \mathscr{T}$ and hence we should have $d_{1}\left(T_{0, t} \nu, \mathscr{T}\right)=0$. Thus, $\ell_{i} / s_{i} \rightarrow 0$. This means that for every $r \geq 1$, taking into account (19), we have

$$
\limsup _{i \rightarrow+\infty} d_{r}\left(T_{x, \ell_{i}} \phi, \mathscr{T}\right)=\limsup _{i \rightarrow+\infty} d_{1}\left(T_{x, r \ell_{i}} \phi, \mathscr{T}\right) \leq \varepsilon,
$$

since $\ell_{i} \leq r \ell_{i} \leq s_{i}$ for $i$ sufficiently large. Since $\varepsilon<1$, we have that $\lambda:=2 /(1+\varepsilon)>1$, and hence, by Remark 2.8, there exists $\tau>1$ such that $F_{\tau r}(\psi) \leq \lambda F_{r}(\psi)$ for every $\psi \in \mathscr{T}$ and for every $r>0$, since $\mathscr{T}$ has a compact basis. Hence, taking (26) into account with $\tau r$ 
instead of $r$, we get that, whenever $r \geq 1$ and $i$ is sufficiently big, there exists $\psi \in \mathscr{T}$ with $F_{\tau r}(\psi)=1$ and

$$
F_{\tau r}\left(\frac{T_{x, \ell_{i}} \phi}{F_{\tau r}\left(T_{x, \ell_{i}} \phi\right)}, \psi\right) \leq \varepsilon / 2 .
$$

As a consequence, whenever $r \geq 1$ and $i$ is sufficiently big, by the triangle inequality for $F$ (cf. Proposition 2.17) and by the fact that $F_{\tau r}(\cdot) \geq F_{r}(\cdot)$, we get that

$$
\frac{F_{r}\left(T_{x, \ell_{i}} \phi\right)}{F_{\tau r}\left(T_{x, \ell_{i}} \phi\right)} \geq F_{r}(\psi)-\varepsilon / 2 \geq \lambda^{-1} F_{\tau r}(\psi)-\varepsilon \geq 1 / 2 .
$$

Hence, iterating, we have shown that there exists $\tau>1$ such that that for every $r \geq 1$ and every $p \in \mathbb{N}$,

$$
\limsup _{i \rightarrow+\infty} \frac{F_{\tau} p_{r}\left(T_{x, \ell_{i}} \phi\right)}{F_{r}\left(T_{x, \ell_{i}} \phi\right)}<+\infty
$$

By the arbitrariness of $p \in \mathbb{N}$ and $r \geq 1$, this implies that we are in a position to apply Proposition 2.16 to the sequence $\frac{T_{x, \ell_{i}} \phi}{F_{1}\left(T_{x, \ell_{i}} \phi\right)}$, which then converges, up to subsequences, to $\widetilde{v} \in \mathscr{T}$ with $F_{1}(\widetilde{v})=1$. But then, by $(18)$,

$$
d_{1}(\widetilde{v}, \mathscr{T})=\lim _{i \rightarrow+\infty} d_{1}\left(T_{x, \ell_{i}} \phi, \mathscr{T}\right) \geq \varepsilon,
$$

that is a contradiction since $d_{1}(\widetilde{v}, \mathscr{T})=0$. Hence we finally have proven (21).

Hence, from (21), we deduce

$$
\limsup _{r \rightarrow 0} \frac{\phi(B(x, 2 r))}{\phi(B(x, r))} \leq \limsup _{r \rightarrow 0} \frac{2 F_{1}\left(T_{x, 4 r} \phi\right)}{2^{-1} F_{1}\left(T_{x, r} \phi\right)} \leq 16 \mathfrak{d}^{-2} q^{4},
$$

whence the conclusion.

Let us now prove a simple consequence of the previous Proposition.

Proposition 2.20 Let $\phi$ be a Radon measure on $\mathbb{G}$ such that for $\phi$-almost every $x \in \mathbb{G}$ we have $\operatorname{Tan}(\phi, x)=\left\{\lambda \mathcal{S}^{h}\llcorner\mathbb{V}(x), \lambda>0\}\right.$ for some homogeneous subgroup $\mathbb{V}(x)$ of Hausdorff dimension $h \in \mathbb{N}$. Then, for $\phi$-almost every $x \in \mathbb{G}$, the measure $T_{x, r} \phi / F_{1}\left(T_{x, r} \phi\right)$ weak* converges to $(h+1) \mathcal{C}^{h}\llcorner\mathbb{V}(x)$.

Proof For $\phi$-almost every $x \in \mathbb{G}$ we have that $\mathfrak{B}(\phi, x)=\left\{(h+1) \mathcal{C}^{h}\llcorner\mathbb{V}(x)\}\right.$, taking into account [4, Proposition 2.12] and Lemma 2.1. Hence $\mathfrak{B}(\phi, x)$ is clearly compact for $\phi$-almost every $x \in \mathbb{G}$, and then $\phi$ is locally asymptotically doubling, due to Proposition 2.19. Hence for every sequence $r_{i} \rightarrow 0$ we can extract a subsequence in $i$ such that $T_{x, r_{i}} \phi / F_{1}\left(T_{x, r_{i}} \phi\right)$ weak* converges to some $v \in \operatorname{Tan}(\phi, x)$, due to the fact that $\phi$ is locally asymptotically doubling and thus the hypothesis of Proposition 2.16 is verified. Since $F_{1}(v)=1$ by continuity of $F_{1}$, we conclude that $v=(h+1) \mathcal{C}^{h}\left\llcorner\mathbb{V}(x)\right.$. Thus, being the sequence $r_{i}$ arbitrary, we obtain the thesis.

The following proposition, which is inspired by [42, 4.4(4)], will be of crucial importance in the proof of the two fundamental results of this section, namely Proposition 2.25, and Proposition 2.26.

Proposition 2.21 Let $0<\sigma<1 / 5$, $\phi$ be a Radon measure on $\mathbb{G}, h \in\{1, \ldots, Q\}$, and $d_{z, t}(\phi, \mathfrak{M}(h,\{\mathbb{V}\})) \leq \sigma^{h+4}$, then

$$
\phi\left(B(y, s) \cap B\left(y \mathbb{V}, \sigma^{2} t /(h+1)\right)\right) \geq(1-5 \sigma)(s / r)^{h} \phi(B(x, r)),
$$


whenever $x, y \in z \mathbb{V} \cap B(z,(1-\sigma) t), \sigma t \leq r \leq(1-\sigma) t-\left\|z^{-1} x\right\|$, and $\sigma t \leq s \leq$ $(1-\sigma) t-\left\|z^{-1} y\right\|$.

Proof The definition of $d_{z, t}(\cdot, \mathfrak{M}(h,\{\mathbb{V}\}))$ implies that

$$
F_{0,1}\left(T_{z, t} \phi / F_{1}\left(T_{z, t} \phi\right),(h+1) \mathcal{C}^{h}\llcorner\mathbb{V}) \leq \sigma^{h+4},\right.
$$

and thus up to redefining $\phi$ we can assume without loss of generality that $z=0, t=1$ and that $F_{1}(\phi)=1$. Thus, let $q:=\sigma^{2} /(h+1), x \in \mathbb{V}$ and $r>0$ as in the hypothesis of the proposition. Define

$$
g(w):=\min \{1, \operatorname{dist}(w, \mathbb{G} \backslash B(x, r+q)) / q\} .
$$

Notice that $B(x, r) \Subset B(0,1)$, and thanks to the assumptions on $\phi$ we infer that, calling $\operatorname{Lip}(g)$ the Lipschitz constant of the function $g$,

$$
\begin{aligned}
& \phi(B(x, r)) \\
& \quad \leq \int g(w) d \phi(w) \leq(h+1) \int g(w) d \mathcal{C}^{h}\left\llcorner\mathbb{V}(w)+\operatorname{Lip}(g) F_{0,1}\left(\phi,(h+1) \mathcal{C}^{h}\llcorner\mathbb{V}(w))\right.\right. \\
& \quad \leq(h+1) \mathcal{C}^{h}\left\llcorner\mathbb{V}(B(x, r+q))+\sigma^{h+4} / q=(h+1)(r+q)^{h}+\sigma^{h+4} / q .\right.
\end{aligned}
$$

With the same argument used above,see [4, Equation (37)], for any $y$ and $s>0$ as in the hypothesis of the proposition one can also show that

$$
(h+1)(s-q)^{h}=(h+1) \mathcal{C}^{h}\left\llcorner\mathbb{V}(B(y, s-q)) \leq \phi(B(y, s) \cap B(\mathbb{V}, q))+\sigma^{h+4} / q .\right.
$$

Thus, putting together (27) and (28) we infer that

$$
\begin{aligned}
& \frac{\phi(B(y, s) \cap B(\mathbb{V}, q))}{\phi(B(x, r))} \\
& \quad \geq \frac{(h+1)(s-q)^{h}-\sigma^{h+4} / q}{(h+1)(r+q)^{h}+\sigma^{h+4} / q}=\left(\frac{s}{r}\right)^{h} \frac{\left(1-\frac{\sigma^{2}}{s(h+1)}\right)^{h}-\frac{\sigma^{h+2}}{s^{h}}}{\left(1+\frac{\sigma^{2}}{r(h+1)}\right)^{h}+\frac{\sigma^{h+2}}{r^{h}}} \\
& \geq\left(\frac{s}{r}\right)^{h} \frac{\left(1-\frac{\sigma}{h+1}\right)^{h}-\sigma^{2}}{\left(1+\frac{\sigma}{h+1}\right)^{h}+\sigma^{2}} \geq\left(\frac{s}{r}\right)^{h} \frac{1-h /(h+1) \sigma-\sigma^{2}}{1+2 h /(h+1) \sigma+\sigma^{2}} \geq\left(\frac{s}{r}\right)^{h} \frac{1-2 \sigma}{1+3 \sigma} \\
& \geq(1-5 \sigma)\left(\frac{s}{r}\right)^{h},
\end{aligned}
$$

where in the third inequality above we are using that $\sigma \leq r$ and $\sigma \leq s$; in the fourth inequality we are using that $(1-\sigma /(h+1))^{h} \geq 1-h /(h+1) \sigma$ by Bernoulli inequality, and $(1+\sigma /(h+1))^{h} \leq 1+2 h /(h+1) \sigma$, which can be easily verified by induction since $2 h \sigma /(h+1) \leq 1$.

Before proving the main results of this section, namely Proposition 2.25, and Proposition 2.26, we now state and prove three measurability results that will play a crucial role in the rest of the paper. Roughly speaking, we prove that when a measure has unique tangents (or unique approximate tangents), the map that associates a point $x \in \mathbb{G}$ to its tangent (or approximate tangent) is measurable. 
Lemma 2.22 Let $\phi$ be a Radon measure such that, for $\phi$-almost every $x \in \mathbb{G}$, there exists $\tau(\phi, x) \in \mathbb{G}(h)$ such that

$$
\operatorname{Tan}(\phi, x)=\left\{\lambda \mathcal{C}^{h}\llcorner\tau(\phi, x): \lambda>0\} .\right.
$$

Then the map $x \mapsto \tau(\phi, x)$ is $\phi$-measurable as a map from $\mathbb{G}$ to $\mathbb{G}(h)$.

Proof First of all, from Proposition 2.19 we get that $\phi$ is locally asymptotically doubling. We let $\left\{\mathbb{V}_{\ell}\right\}_{\ell \in \mathbb{N}}$ be a countable dense set in $\mathbb{G}(h)$ that exists thanks to the compactness of the Grassmanian, see Proposition 2.7. Furthermore, for any $r \in(0,1) \cap \mathbb{Q}$ any $\varepsilon>0$, and any $\ell \in \mathbb{N}$ we define the function

$$
\begin{aligned}
& f_{r, \ell, \varepsilon}(x):=\phi(B(x, r))^{-1} \phi\left(\left\{w \in B(x, r): \operatorname{dist}\left(x^{-1} w, \mathbb{V}_{\ell}\right)\right.\right. \\
& \left.\left.\quad \geq \varepsilon\left\|x^{-1} w\right\|\right\}\right)=: \phi(B(x, r))^{-1} \phi(I(x, r, \ell, \varepsilon)),
\end{aligned}
$$

when $\phi(B(x, r))>0$ and we set it to be $+\infty$ if $\phi(B(x, r))=0$. We claim that the functions $f_{r, \ell, \varepsilon}$ are upper semicontinuous. Let $\left\{x_{i}\right\}_{i \in \mathbb{N}}$ be a sequence of points converging to some $x \in \mathbb{G}$. If $\phi(B(x, r))=0$ the upper semicontinuity on the sequence $\left\{x_{i}\right\}_{i \in \mathbb{N}}$ is trivially verified by definition of $f_{r, \ell, \varepsilon}$. So let us assume without loss of generality that $\phi(B(x, r))>0$. Since $x_{i} \rightarrow x$ and $\phi$ is a Radon measure we have $\phi(B(x, r))=\lim _{i} \phi\left(B\left(x_{i}, r\right)\right)$, and then we can assume withouot loss of generality that $\phi\left(B\left(x_{i}, r\right)\right)>0$ for every $i$.

Since the sets $I\left(x_{i}, r, \ell, \varepsilon\right)$ are contained in $B(x, 2)$ provided $i$ is sufficiently big, we infer thanks to Fatou's Lemma that

$$
\begin{aligned}
& \limsup _{i \rightarrow+\infty} f_{r, \ell, \varepsilon}\left(x_{i}\right)=\limsup _{i \rightarrow+\infty} \phi\left(B\left(x_{i}, r\right)\right)^{-1} \int \chi_{I\left(x_{i}, r, \ell, \varepsilon\right)}(z) d \phi(z) \\
& \quad \leq \phi(B(x, r))^{-1} \int \limsup _{i \rightarrow+\infty} \chi_{I\left(x_{i}, r, \ell, \varepsilon\right)}(z) d \phi(z) .
\end{aligned}
$$

Furthermore, since $x_{i} \rightarrow x$ and the sets $I\left(x_{i}, r, \ell, \varepsilon\right)$ and $I(x, r, \ell, \varepsilon)$ are closed, we have

$$
\begin{aligned}
& \limsup _{i \rightarrow+\infty} \chi_{I\left(x_{i}, r, \ell, \varepsilon\right)} \\
& \quad=\chi_{\lim \sup _{i \rightarrow+\infty} I\left(x_{i}, r, \ell, \varepsilon\right)} \leq \chi_{I(x, r, \ell, \varepsilon)},
\end{aligned}
$$

where the first equality is true in general. Then, from (29), we infer that

$$
\begin{aligned}
& \limsup _{i \rightarrow \infty} f_{r, \ell, \varepsilon}\left(x_{i}\right) \leq \phi(B(x, r))^{-1} \int \limsup _{i \rightarrow+\infty} \chi_{I\left(x_{i}, r, \ell, \varepsilon\right)}(z) d \phi(z) \\
& \leq \phi(B(x, r))^{-1} \int \chi_{I(x, r, \ell, \varepsilon)}(z) d \phi(z)=f_{r, \ell, \varepsilon}(x),
\end{aligned}
$$

and this concludes the proof that $f_{r, \ell, \varepsilon}$ is upper semicontinuous. This shows that for every $\ell \in \mathbb{N}$ and $\varepsilon>0$, the function

$$
f_{\ell, \varepsilon}:=\liminf _{r \in \mathbb{Q} \cap(0,1), r \rightarrow 0} f_{r, \ell, \varepsilon}
$$

is $\phi$-measurable. Hence also $\widetilde{f}_{\ell, \varepsilon}:=\sup _{\widetilde{\varepsilon} \in \mathbb{Q}, \widetilde{\varepsilon}>\varepsilon} f_{\ell, \varepsilon}$ is $\phi$-measurable. As a consequence, since $\operatorname{Tan}(\phi, x)=\left\{\lambda \mathcal{C}^{h}\llcorner\tau(\phi, x): \lambda>0\}\right.$ for $\phi$-almost every $x \in \mathbb{G}$, we infer that the set

$$
\begin{aligned}
B_{\ell, \varepsilon} & :=\left\{x \in \mathbb{G}: \tilde{f}_{\ell, \varepsilon}(x)=0\right\} \cap\{x \in \mathbb{G}: \text { there exists } \tau(\phi, x)\} \\
& =\left\{x \in \mathbb{G}: \tau(\phi, x) \subseteq C_{\mathbb{V}_{\ell}}(\varepsilon)\right\},
\end{aligned}
$$


is $\phi$-measurable as well. Let us justify the last equality in the previous line. If $\widetilde{f}_{\ell, \varepsilon}(x)=0$, then $f_{\ell, \widetilde{\varepsilon}}(x)=0$ for every $\widetilde{\varepsilon}>\varepsilon, \widetilde{\varepsilon} \in \mathbb{Q}$. Hence, arguing as in $[4$, Proof of Proposition 5.5], in particular as in the lines slightly above [4, Equation (109)], we get $\tau(\phi, x) \subseteq C_{\mathbb{V}_{\ell}}(\widetilde{\varepsilon})$ for every $\widetilde{\varepsilon} \in \mathbb{Q}$ and $\widetilde{\varepsilon}>\varepsilon$. Let us explain this with further details. We first get that there exist $r_{i} \rightarrow 0$ such that $f_{r_{i}, \ell, \widetilde{\varepsilon}}(x) \rightarrow_{i} 0$. Since $\phi$ is locally asymptotically doubling, thanks to Proposition 2.16 we deduce that $\phi\left(B\left(x, r_{i}\right)\right)^{-1} T_{x, r_{i}} \phi$ converges, up to subsequences, to some tangent measure $v \in \operatorname{Tan}(\phi, x)$, and then from the hypothesis we have $v=\lambda \mathcal{C}^{h}\llcorner\tau(\phi, x)$, for some $\lambda>0$. Then the same computations in the two displayed equations before [4, Equation (109)] give the sought conclusion. Taking $\widetilde{\varepsilon} \rightarrow \varepsilon$ we get the first inclusion of (30). On the other hand, if $\tau(\phi, x) \subseteq C_{\mathbb{V}_{\ell}}(\varepsilon)$, we get that $\tau(\phi, x) \subseteq\left\{w \in \mathbb{G}: \operatorname{dist}\left(w, \mathbb{V}_{\ell}\right)<\widetilde{\varepsilon}\|w\|\right\}$ for every $\widetilde{\varepsilon}>\varepsilon$. Hence we can argue as in [4, Equation (111)] in order to obtain that $f_{\ell, \widetilde{\varepsilon}}(x)=0$ for every $\widetilde{\varepsilon}>\varepsilon$ and then passing to the limit as $\widetilde{\varepsilon} \rightarrow \varepsilon$ we get the sought conclusion.

In order to prove that the map $x \mapsto \tau(\phi, x)$ is $\phi$-measurable, it suffices to check that the for any open $\Omega \subseteq \mathbb{G}(h)$ we have that $\tau^{-1}(\Omega)$ is $\phi$-measurable. To show this we note that, thanks to [4, Lemma 2.15], there is a sequence of radii $r_{k}>0$ such that

$$
\Omega=\bigcup_{\substack{k \in \mathbb{N} \\ \mathbb{V}_{k} \in \Omega}}\left\{\mathbb{W} \in \mathbb{G}(h): \mathbb{W} \subseteq C_{\mathbb{V}_{k}}\left(r_{k}\right)\right\}
$$

This implies that, up to $\phi$-null sets, $\tau^{-1}(\Omega)=\bigcup_{k \in \mathbb{N}} B_{k, r_{k}}$, which thanks to the above discussion is a $\phi$-measurable set.

Lemma 2.23 Let $\phi$ be a $\mathscr{P}_{h}$-rectifiable measure. Denote $\tau(\phi, x)$ to be the unique element of $\mathbb{G}(h)$, that exists $\phi$-almost everywhere by definition, for which

$$
\operatorname{Tan}_{h}(\phi, x) \subseteq\left\{\lambda \mathcal{C}^{h}\llcorner\tau(\phi, x): \lambda>0\} .\right.
$$

Then the map $x \mapsto \tau(\phi, x)$ is $\phi$-measurable as a map from $\mathbb{G}$ to $\mathbb{G}(h)$.

Proof From a routine argument (cf. [38, Remark 14.4(3)]), we get that $\operatorname{Tan}(\phi, x)=$ $\left\{\lambda \mathcal{C}^{h}\llcorner\tau(\phi, x)\}\right.$ for $\phi$-almost every $x \in \mathbb{G}$. Hence we can apply Lemma 2.22

The proof of the following lemma follows as the ones above. We omit the details.

Lemma 2.24 Suppose d is a homogeneous left-invariant metric on $\mathbb{G}$, let $E$ be a Borel set of finite $\mathcal{S}_{d}^{h}$-measure, and suppose that for $\mathcal{S}_{d}^{h}$-almost every $x \in E$ there exists $\mathbb{V}(x) \in \mathbb{G}(h)$ for which for any $0<\varepsilon<1$ and any $0<\beta<1$ there exist $a \rho(x, \varepsilon, \beta)>0$ such that

$$
\mathcal{S}_{d}^{h}\left\llcorner E\left(B_{d}(x, r) \backslash x C_{\mathbb{V}(x), d}(\beta)\right) \leq \varepsilon \mathcal{S}_{d}^{h}\left\llcorner E\left(B_{d}(x, r)\right),\right.\right.
$$

for any $0<r<\rho(x, \varepsilon, \beta)$. Then the map $x \mapsto \mathbb{V}(x)$ from $E$ to $\mathbb{G}(h)$ is $\mathcal{S}_{d}^{h}\llcorner E$-measurable.

Notice that the previous statement could be also obtained arguing as in [39, Proposition 3.9], after having noticed that, since $2^{-h} \leq \Theta_{d}^{h, *}\left(\mathcal{S}_{d}^{h}\llcorner E, x) \leq 1\right.$ for $\mathcal{S}_{d}^{h}\llcorner E$-almost every $x \in \mathbb{G}$ due to [15, 2.10.19(1) and 2.10.19(5)], the condition (31) is equivalent to asking that $\mathbb{V}(x)$ is an approximate tangent plane to $E$ at $x$ in the sense of [39, Equation (3.2)].

Remark 2.9 The results in Lemma 2.22, Lemma 2.23, and Proposition 2.24 are readily true also when we allow $\tau(\phi, x)$ (or $\mathbb{V}(x))$ to be in some Borel subset of $\mathbb{G}(h)$.

Proposition 2.25 Suppose $\phi$ is a Radon measure on $\mathbb{G}$ such that, for $\phi$-almost every $x \in \mathbb{G}$, we have $\operatorname{Tan}(\phi, x)=\left\{\lambda \mathcal{S}^{h}\llcorner\mathbb{V}(x): \lambda>0\}\right.$ for some $\mathbb{V}(x) \in \mathbb{G}(h)$. Then, for every $\alpha \in(0,1)$ there exist $\left\{\mathbb{V}_{i}\right\}_{i \in \mathbb{N}} \subseteq \mathbb{G}(h)$, and a family of compact $C_{\mathbb{V}_{i}}(\alpha)$-sets $\left\{\Gamma_{i}\right\}_{i \in \mathbb{N}}$ such that

$$
\phi\left(\mathbb{G} \backslash \cup_{i \in \mathbb{N}} \Gamma_{i}\right)=0 .
$$


Proof First of all, by Proposition 2.19, the measure $\phi$ is locally asymptotically doubling. Up to restricting $\phi$ to closed balls and by using the locality of tangents in Proposition 2.6 and Lebesgue Theorem in Proposition 2.6, we may assume that $\phi$ is supported on a compact set $K$ and that it is still locally asymptotically doubling. Let $S$ be dense countable subset of $\left(\mathbb{G}(h), d_{\mathbb{G}}\right)$, that exists thanks to Proposition 2.7. Thanks to [4, Proposition 2.29], we infer that also the countable set $\left\{(h+1) \mathcal{C}^{h}\llcorner\mathbb{W}: \mathbb{W} \in S\}\right.$ is dense in the metric space $\left(\left\{(h+1) \mathcal{C}^{h}\llcorner\mathbb{V}: \mathbb{V} \in \mathbb{G}(h)\}, F_{0,1}\right)\right.$.

Let us now fix $\mathfrak{e}<1 / 10, \sigma<1 / 100(\mathfrak{e} /(3(1+\mathfrak{e})))^{h}, \mathbb{V} \in S$ and let us denote

$$
K_{\mathbb{V}}:=\left\{x \in K: F_{0,1}\left((h+1) \mathcal{C}^{h}\left\llcorner\mathbb{V},(h+1) \mathcal{C}^{h}\llcorner\mathbb{V}(x))<\sigma^{h+4}\right\},\right.\right.
$$

where $\mathbb{V}(x) \in \mathbb{G}(h)$ is such that $\operatorname{Tan}(\phi, x)=\left\{\lambda \mathcal{S}^{h}\llcorner\mathbb{V}(x), \lambda>0\}\right.$. Since $\left\{(h+1) \mathcal{C}^{h}\llcorner\mathbb{W}\right.$ : $\mathbb{W} \in S\}$ is dense in the metric space $\left(\left\{(h+1) \mathcal{C}^{h}\llcorner\mathbb{V}: \mathbb{V} \in \mathbb{G}(h)\}, F_{0,1}\right)\right.$ we conclude that $K=\cup_{\mathbb{V} \in S} K_{\mathbb{V}}$. By Lemma 2.22, one gets that $K_{\mathbb{V}}$ is $\phi$-measurable for every $\mathbb{V} \in S$. Thus by Proposition 2.6, we can assume without loss of generality that $\phi$ is locally asymptotically doubling and supported on $K_{\mathbb{V}}$ for some $\mathbb{V} \in S$, which from now on we fix.

We now claim that for $\phi$-almost every $x \in \mathbb{G}$ the following holds

$$
\lim _{r \rightarrow 0^{+}} d_{x, r}(\phi, \mathfrak{M}(h,\{\mathbb{V}\}))=F_{0,1}\left(( h + 1 ) \mathcal { C } ^ { h } \left\llcorner\mathbb{V},(h+1) \mathcal{C}^{h}\llcorner\mathbb{V}(x))\right.\right.
$$

Indeed, for $\phi$-almost every $x \in \mathbb{G}$ the measure $T_{x, r} \phi / F_{1}\left(T_{x, r} \phi\right)$ converges to $(h+1) \mathcal{C}^{h}\llcorner\mathbb{V}(x)$ as $r \rightarrow 0^{+}$, see Proposition 2.20 and thus, from the definition of $d_{x, r}$, we get that

$$
d_{x, r}(\phi, \mathfrak{M}(h,\{\mathbb{V}\}))=F_{0,1}\left(T_{x, r} \phi / F_{1}\left(T_{x, r} \phi\right),(h+1) \mathcal{C}^{h}\llcorner\mathbb{V}),\right.
$$

from which we deduce the claim (32) by using the previous convergence and the continuity of $F_{0,1}$, see, e.g., [40, Proposition 1.10] or Proposition2.16. Moreover, the function $x \rightarrow$ $d_{x, r}(\phi, \mathfrak{M}(h,\{\mathbb{V}\}))$ is continuous in $x$ for every $r>0$. Indeed, by (33) and the continuity of $F_{0,1}$, it is sufficent to see that, for every $r>0$, the map $x \rightarrow T_{x, r} \phi / F_{1}\left(T_{x, r} \phi\right)$ is continuous from $\mathbb{G}$ to the space of Radon measures equipped with the weak* convergence, which is clear again by the continuity of $F_{1}(\cdot)$ and by the continuity of the map $x \rightarrow T_{x, r} \phi$, which is readily verified (see, e.g., the computations at the end of [40, page 22]). Hence, by using SeveriniEgoroff Theorem, we can assume without loss of generality that $\phi$ is supported on a compact set $E$ such that $\operatorname{diam}(E)<s$ and such that $d_{x, r}(\phi, \mathfrak{M}(h,\{\mathbb{V}\}))<\sigma^{h+4}$ whenever $x \in E$ and $r \in(0,400(h+1) s)$. Let us now fix $\tilde{x}, \tilde{y} \in E$ and denote $a:=d(\tilde{x}, \tilde{y}), \tilde{t}:=2 a(1+\mathfrak{e})$, $\tilde{r}:=a(1+\mathfrak{e})$ and $\tilde{s}:=a \mathfrak{e}$.

Let us apply Proposition 2.21 first with the choices $x=y=z=\tilde{y}, s=r=\widetilde{r}, t=\tilde{t}$ and $\sigma$ as above, that yields

$$
\phi\left(B(\tilde{y}, \widetilde{r}) \cap B\left(\widetilde{y} \mathbb{V}, \sigma^{2} \widetilde{t} /(h+1)\right)\right) \geq(1-5 \sigma) \phi(B(\tilde{y}, \widetilde{r})),
$$

and secondly with $x=y=z=\tilde{x}, r=\widetilde{r}+a, s=\widetilde{s}, t=3 a(1+\mathfrak{e})$ and $\sigma$, we get

$$
\phi\left(B(\tilde{x}, \widetilde{s}) \cap B\left(\widetilde{x} \mathbb{V}, \sigma^{2} \cdot 3 a(1+\mathfrak{e}) /(h+1)\right)\right) \geq(1-5 \sigma)(\widetilde{s} /(\widetilde{r}+a))^{h} \phi(B(\tilde{x}, \tilde{r}+a)) .
$$


Putting together (34) and (35), we conclude that

$$
\begin{aligned}
\phi & \left(B(\tilde{y}, \widetilde{r}) \backslash B\left(\widetilde{y} \mathbb{V}, 2 a \sigma^{2}(1+\mathfrak{e}) /(h+1)\right)\right) \\
& =\phi(B(\tilde{y}, \widetilde{r}))-\phi\left(B(\tilde{y}, \widetilde{r}) \cap B\left(\widetilde{y} \mathbb{V}, 2 a \sigma^{2}(1+\mathfrak{e}) /(h+1)\right)\right) \\
& \leq 5 \sigma \phi(B(\tilde{y}, \widetilde{r})) \\
& \leq 5 \sigma \phi(B(\tilde{x}, \widetilde{r}+a)) \\
& \leq \frac{5 \sigma}{1-5 \sigma}\left(\frac{2(1+\mathfrak{e})}{\mathfrak{e}}\right)^{h} \phi(B(\tilde{x}, \widetilde{s}))
\end{aligned}
$$

If by contradiction $\left.B(\tilde{x}, \mathfrak{e} a) \cap B\left(\tilde{y} \mathbb{V}, 2 a \sigma^{2}(1+\mathfrak{e}) /(h+1)\right)\right)=\emptyset$ then from (36) and the fact that $B(\tilde{x}, \widetilde{s}) \subseteq B(\tilde{y}, \widetilde{r})$, we infer

$$
\phi(B(\widetilde{x}, \widetilde{s})) \leq \frac{5 \sigma}{1-5 \sigma}\left(\frac{2(1+\mathfrak{e})}{\mathfrak{e}}\right)^{h} \phi(B(\widetilde{x}, \widetilde{s})),
$$

that is in contradiction thanks with the choice of $\sigma$. Hence, for every $\tilde{x}, \tilde{y} \in E$ we have that $\left.B(\tilde{x}, \mathfrak{e} a) \cap B\left(\widetilde{y} \mathbb{V}, 2 a \sigma^{2}(1+\mathfrak{e}) /(h+1)\right)\right) \neq \emptyset$ and thus $d(\widetilde{x}, \widetilde{y} \mathbb{V}) \leq a\left(\mathfrak{e}+2 \sigma^{2}(1+\mathfrak{e}) /(h+1)\right)=$ $d(\tilde{x}, \tilde{y})\left(\mathfrak{e}+2 \sigma^{2}(1+\mathfrak{e}) /(h+1)\right)$. Hence, the compact set $E$ is a $C_{\mathbb{V}}\left(\mathfrak{e}+2 \sigma^{2}(1+\mathfrak{e}) /(h+1)\right)-$ set. Since it is clear that, for any given $\alpha>0, \sigma$ and $\mathfrak{e}$ can be chosen small enough in order to have $\mathfrak{e}+2 \sigma^{2}(1+\mathfrak{e}) /(h+1)<\alpha$, the proof is thus concluded.

In the case the tangents are complemented we can give the following improvement of the latter Proposition.

Proposition 2.26 Let $1 \leq h \leq Q$ be a natural number. There exist $\left\{\mathbb{V}_{i}\right\}_{i \in \mathbb{N}} \subseteq \mathbb{G}_{c}(h)$ and $\mathbb{L}_{i}$ complementary subgroups of $\mathbb{V}_{i}$ such that the following holds.

Suppose $\phi$ is a Radon measure on $\mathbb{G}$ such that, for $\phi$-almost every $x \in \mathbb{G}$, we have $\operatorname{Tan}(\phi, x)=\left\{\lambda \mathcal{S}^{h}\llcorner\mathbb{V}(x): \lambda>0\}\right.$ for some $\mathbb{V}(x) \in \mathbb{G}_{c}(h)$. Then, for every $\alpha \in(0,1)$ there exists a family of compact sets $\left\{\Gamma_{i}\right\}_{i \in \mathbb{N}}$ such that

$$
\phi\left(\mathbb{G} \backslash \cup_{i \in \mathbb{N}} \Gamma_{i}\right)=0,
$$

and, for every $i \in \mathbb{N}, \Gamma_{i}$ is a compact intrinsic Lipschitz graph, which is also a $C_{\mathbb{V}_{i}}(\alpha)$-set, of a map $\varphi_{i}: A_{i} \subseteq \mathbb{V}_{i} \rightarrow \mathbb{L}_{i}$, where $A_{i}$ is compact.

Proof The proof follows exactly the same lines as the proof of Proposition 2.25, so we just sketch it underlying the main differences. For every $\ell \in \mathbb{N}$, with $\ell \geq 2$, let us define

$$
\mathbb{G}_{c}(h, \ell):=\left\{\mathbb{V} \in \mathbb{G}_{c}(h): \exists \mathbb{L} \text { complement of } \mathbb{V} \text { s.t. } 1 / \ell<\varepsilon_{1}(\mathbb{V}, \mathbb{L}) \leq 1 /(\ell-1)\right\} .
$$

Observe that Proposition 2.7 implies that $\mathbb{G}_{c}(h, \ell)$ is separable for any $\ell \in \mathbb{N}$, since $\mathbb{G}_{c}(h, \ell) \subseteq \mathbb{G}(h)$ and $\left(\mathbb{G}(h), d_{\mathbb{G}}\right)$ is a compact metric space. Let

$$
\mathscr{D}_{\ell}:=\left\{\mathbb{V}_{i, \ell}\right\}_{i \in \mathbb{N}},
$$

be a countable dense subset of $\mathbb{G}_{c}(h, \ell)$ and

$$
\text { for all } i \in \mathbb{N} \text {, choose a complement } \mathbb{L}_{i, \ell} \text { of } \mathbb{V}_{i, \ell} \text { s.t. } 1 / \ell<\varepsilon_{1}\left(\mathbb{V}_{i, \ell}, \mathbb{L}_{i, \ell}\right) \leq 1 /(\ell-1) \text {. }
$$

Now, let $S:=\left\{\mathbb{V}_{i, \ell}\right\}_{i, \ell \in \mathbb{N}}$, which is a dense countable subset of $\left(\mathbb{G}_{c}(h), d_{\mathbb{G}}\right)$ thanks to the definition given above. As in the above proposition we infer that also the countable set $\left\{(h+1) \mathcal{C}^{h}\llcorner\mathbb{W}: \mathbb{W} \in S\}\right.$ is dense in the metric space $\left(\left\{(h+1) \mathcal{C}^{h}\left\llcorner\mathbb{V}: \mathbb{V} \in \mathbb{G}_{c}(h)\right\}, F_{0,1}\right)\right.$. Let us now fix, for every $\ell \in \mathbb{N}, \mathfrak{e}_{\ell}<\min \{1 / 10,1 /(2 \ell), \alpha / 2\}$, where $\alpha$ is as in the statement, 
and $\sigma_{\ell}<\min \left\{1 / 100\left(\mathfrak{e}_{\ell} /\left(3\left(1+\mathfrak{e}_{\ell}\right)\right)\right)^{h}, \sigma_{\ell}^{\prime}\right\}$, where $\sigma_{\ell}^{\prime}$ is chosen small enough such that $\mathfrak{e}_{\ell}+2\left(\sigma_{\ell}^{\prime}\right)^{2}\left(1+\mathfrak{e}_{\ell}\right) /(h+1)<\min \{\alpha, 1 / \ell\}$. Moreover, for every $\mathbb{V}_{i, \ell} \in \mathscr{D}_{\ell}$, let us denote

$$
K_{\mathbb{V}_{i, \ell}}:=\left\{x \in K: F_{0,1}\left((h+1) \mathcal{C}^{h}\left\llcorner\mathbb{V}_{i, \ell},(h+1) \mathcal{C}^{h}\llcorner\mathbb{V}(x))<\sigma_{\ell}^{h+4}\right\},\right.\right.
$$

where $\mathbb{V}(x)$ is the element of $\mathbb{G}(h)$ for which $\operatorname{Tan}(\phi, x)=\left\{\lambda \mathcal{S}^{h}\llcorner\mathbb{V}(x), \lambda>0\}\right.$. Arguing as in the above proposition, being $K$ the compact set on which we can assume $\phi$ is supported without loss of generality, we have $K=\cup_{\ell \in \mathbb{N}} \cup_{\mathbb{V}_{i, \ell} \in \mathscr{D}_{\ell}} K_{\mathbb{V}_{i, \ell}}$. Hence, we can assume without loss of generality that $\phi$ is supported on $K_{\mathbb{V}_{i, \ell}}$ for some $\mathbb{V}_{i, \ell}$. The computations in the proof of the above proposition can be repeated substituting $\sigma_{\ell}$ with $\sigma$ accordingly, allowing us to conclude that $\phi$-almost every $K_{\mathbb{V}_{i, \ell}}$ can be covered by compact sets that are $C_{\mathbb{V}_{i, \ell}}\left(\mathfrak{e}_{\ell}+\right.$ $\left.2\left(\sigma_{\ell}\right)^{2}\left(1+\mathfrak{e}_{\ell}\right) /(h+1)\right)$. By the very choice of $\sigma_{\ell}^{\prime}$ this implies that the latter compact sets are $C_{\mathbb{V}_{i, \ell}}(\min \{\alpha, 1 / \ell\})$-sets, and since $1 / \ell<\varepsilon_{1}\left(\mathbb{V}_{i, \ell}, \mathbb{L}_{i, \ell}\right)$, we also conclude that they are graphs according to the splitting $\mathbb{G}=\mathbb{V}_{i, \ell} \cdot \mathbb{L}_{i, \ell}$, see Proposition 2.13.

\section{From flat tangents to $\mathscr{P}$-rectifiability}

In this section we first prove that, in an arbitrary Carnot group, having flat (complemented) tangent measures à la Preiss implies being $\mathscr{P}$-rectifiable, see Theorem 3.7. Then we will prove a rectifiable criterion, see Proposition 3.9, which will allow us to complete the proof of Theorem 1.1.

In this section a Carnot group $\mathbb{G}$ will be fixed, along with a left-invariant homogeneous distance on it, that sometimes will be understood. Throughout this section we assume that $\mathbb{V} \in \mathbb{G}_{c}(h)$ and that $\mathbb{V} \mathbb{L}=\mathbb{G}$. In this chapter whenever we deal with $C_{\mathbb{V}}(\alpha)$-sets we are tacitly assuming that $\alpha \leq \varepsilon_{1}(\mathbb{V}, \mathbb{L})$.

\subsection{From flat Preiss's tangents to $\mathscr{P}$-rectifiability}

We are going to prove that whenever a measure has flat (complemented) tangents á la Preiss, then it is $\mathscr{P}$-rectifiable. Throughout this section we assume that $\mathbb{V} \in \mathbb{G}_{c}(h)$ and that $\mathbb{L}$ is a complementary subgroup of $\mathbb{V}$. Let us begin with a proposition that roughly tells us the following. If $\Gamma$ is a compact $C_{\mathbb{V}}(\alpha)$-set with $\alpha \leq \varepsilon_{1}(\mathbb{V}, \mathbb{L})$, and moreover we know that the measure $\mathcal{S}^{h}\llcorner\Gamma$ is locally asymptotically doubling, hence $\Gamma$ has big projections on $\mathbb{V}$. This will allow us to prove that the lower $h$-density of $\mathcal{S}^{h}\llcorner\Gamma$ is positive almost everywhere, see Proposition 3.6. The latter conclusion eventually leads to the following result: if a set has flat (complemented) Preiss's tangents, then it is $\mathscr{P}^{c}$-rectifiable, see Theorem 3.7.

Let us start by recalling two results from [4] and proving an adaption of [4, Proposition 4.6] to our context.

Lemma 3.1 ([4, Lemma 4.2]) Suppose d is a homogeneous left-invariant metric on $\mathbb{G}$. Then, there exists an $A:=A(d, \mathbb{V}, \mathbb{L})>1$ such that for any $w \in B_{d}(0,1 / 5 A)$, any $y \in \partial B_{d}(0,1) \cap$ $C_{\mathbb{V}, d}\left(\varepsilon_{1}(d, \mathbb{V}, \mathbb{L})\right)$ and any $z \in B_{d}(y, 1 / 5 A)$, we have $w^{-1} z \notin \mathbb{L}$.

Proposition 3.2 ([4, Proposition 4.3]) Suppose d is a homogeneous left-invariant metric on $\mathbb{G}$. Let us fix $\alpha<\varepsilon_{1}(d, \mathbb{V}, \mathbb{L})$ and suppose $\Gamma$ is a $C_{\mathbb{V}, d}(\alpha)$-set. For any $x \in \Gamma$ let $\rho(x)$ to be the biggest number satisfying the following condition. For any $0<r<\rho(x)$ and any $y \in B(x, r) \cap \Gamma$ we have

$$
P_{\mathbb{V}}\left(B_{d}(x, r)\right) \cap P_{\mathbb{V}}\left(B_{d}(y, s)\right)=\emptyset \text { for any } r, s<d(x, y) / 5 A,
$$


where $A=A(d, \mathbb{V}, \mathbb{L})$ is the constant yielded by Lemma 3.1. Then, the function $x \mapsto \rho(x)$ is positive everywhere on $\Gamma$ and upper semicontinuous.

Proposition 3.3 Let $\alpha \leq \varepsilon_{1}(\mathbb{V}, \mathbb{L})$ and suppose $\Gamma$ is a compact $C_{\mathbb{V}}(\alpha)$-set of $\mathcal{S}^{h}$-finite measure such that for $\mathcal{S}^{h}\llcorner\Gamma$-almost every $x \in \mathbb{G}$ we have

$$
\limsup _{r \rightarrow 0} \frac{\mathcal{S}^{h}\llcorner\Gamma(B(x, 2 r))}{\mathcal{S}^{h}\llcorner\Gamma(B(x, r))}<\infty .
$$

Then, there exists a constant $C_{3}:=C_{3}(h, A)>0$ such that for $\mathcal{S}^{h}$-almost every $x \in \Gamma$ there exists an infinitesimal sequence $\left\{\ell_{i}(x)\right\}_{i \in \mathbb{N}}$ such that for any $i \in \mathbb{N}$ we have

$$
\mathcal{S}^{h}\left(P_{\mathbb{V}}\left(\Gamma \cap B\left(x, \ell_{i}(x)\right)\right)\right)>C_{3} \ell_{i}(x)^{h}
$$

Proof We will just sketch the proof, that is an adaptation of [4, Proposition 4.6] and we refer the reader to [4, Proposition 4.6] for the missing details. Let $N \in \mathbb{N}$ be the unique natural number for which $5^{N-2} \leq A<5^{N-1}$, where $A$ is as in Lemma 3.1. Notice that, since $2^{-h} \leq \Theta^{h, *}\left(\mathcal{S}^{h}\llcorner\Gamma, x) \leq 1\right.$ for $\mathcal{S}^{h}\llcorner\Gamma$-almost every $x \in \mathbb{G}$ (cf. [15, 2.10.19(1) and 2.10.19(5)]), hence, for $\mathcal{S}^{h}\llcorner\Gamma$-almost every $x \in \mathbb{G}$ there exists an infinitesimal sequence $\left\{\ell_{i}(x)\right\}_{i \in \mathbb{N}}$ such that

$$
\frac{1}{2^{h+1}} \leq \frac{\mathcal{S}^{h}\left(\Gamma \cap B\left(x, \ell_{i}(x)\right)\right)}{\ell_{i}(x)^{h}} \leq 2 .
$$

Thus, for any $k \in \mathbb{N}$ and $0<\delta<1 / 2$ we define the following sets

$$
\begin{aligned}
A(k) & :=\{x \in \Gamma: \rho(x)>1 / k\}, \\
\mathscr{D}(k) & :=\left\{x \in A(k): \lim _{r \rightarrow 0} \frac{\mathcal{S}^{h}(B(x, r) \cap A(k))}{\mathcal{S}^{h}(B(x, r) \cap \Gamma)}=1\right\}, \\
\mathscr{F}_{\delta}(k) & :=\left\{B(x, r): x \in \mathscr{D}(k), r \leq \frac{\min \left\{k^{-1}, \delta\right\}}{1000 A} \text { and } \frac{1}{2^{h+1}} \leq \frac{\mathcal{S}^{h}\left\llcorner\Gamma\left(B\left(x, 5^{N+1} r\right)\right)\right.}{\left(5^{N+1} r\right)^{h}} \leq 2\right\},
\end{aligned}
$$

where $\rho(x)$ is the number defined in Proposition 3.2. First of all notice that, thanks to (39), $\mathscr{F}_{\delta}(k)$ is a fine covering of $\mathcal{S}^{h}\llcorner\Gamma$-almost all $\mathscr{D}(k)$. Furthermore, for any $k$ the sets $A(k)$ are Borel since thanks to Proposition 3.2, the function $\rho$ is upper semicontinuous and, since by assumption $\mathcal{S}^{h}\left\llcorner\Gamma\right.$ is locally asymptotically doubling, we also know that $\mathcal{S}^{h}\llcorner\Gamma(A(k) \backslash \mathscr{D}(k))=$ 0. Finally, from Proposition 3.2 we infer that $\mathcal{S}^{h}\left(\Gamma \backslash \cup_{k=1}^{+\infty} A(k)\right)=0$. Let us apply [4, Lemma 4.5] to $N$ and $\mathscr{F}_{\delta}(k)$ and we obtain the disjoint subfamily $\mathscr{G}_{\delta}(k)$ of $\mathscr{F}_{\delta}(k)$ such that

$(\alpha)$ for any $B, B^{\prime} \in \mathscr{G}_{\delta}(k)$ we have that $5^{N} B \cap 5^{N} B^{\prime}=\emptyset$,

( $\beta) \bigcup_{B \in \mathscr{F}_{\delta}(k)} B \subseteq \bigcup_{B \in \mathscr{G}_{\delta}(k)} 5^{N+1} B$.

Throughout the rest of the proof we fix a $w \in \mathscr{D}(k)$ such that there exists a sequence $\left\{\ell_{i}(w)\right\}_{i \in \mathbb{N}}$ satisfying (39), $\ell_{i}(w) \leq \min \left\{k^{-1}, \delta\right\} / 8$, and

$$
\frac{\mathcal{S}^{h}\left\llcorner\mathscr{D}(k)\left(B\left(w, \ell_{i}(w)\right)\right)\right.}{\mathcal{S}^{h}\left\llcorner\Gamma\left(B\left(w, \ell_{i}(w)\right)\right)\right.} \geq \frac{1}{2} \quad \text { for any } i \in \mathbb{N},
$$

where the inequality follows from the fact that $\mathcal{S}^{h}\llcorner\Gamma$-almost every point of $\mathscr{D}(k)$ has density one with respect to the locally asymptotically doubling measure $\mathcal{S}^{h}\llcorner\Gamma$. Notice that, according to the previous discussion, the previous choice on $w$ is made in a set of full $\mathcal{S}^{h}\llcorner\Gamma$-measure, so that if we prove the estimate (38) with such a $w$ we are done. For the ease of notation we 
continue the proof fixing the radius $\ell_{i}(w)=R$. We stress that the forthcoming estimates are verified also for any $\ell_{i}(w)$. As in [4, Proposition 4.6], one can prove that for any couple of closed balls $B(x, r), B(y, s) \in \mathscr{G}_{\delta}(k)$ such that $B(w, R)$ intersects both $B\left(x, 5^{N+1} r\right)$ and $B\left(y, 5^{N+1} s\right)$, we have

$$
P_{\mathbb{V}}(B(x, r)) \cap P_{\mathbb{V}}(B(y, s))=\emptyset .
$$

In order to proceed with the conclusion of the proof, let us define

$$
\begin{aligned}
\mathscr{F}_{\delta}(w, R) & :=\left\{B \in \mathscr{F}_{\delta}(k): 5^{N+1} B \cap B(w, R) \cap \mathscr{D}(k) \neq \emptyset\right\}, \\
\mathscr{G}_{\delta}(w, R) & :=\left\{B \in \mathscr{G}_{\delta}(k): 5^{N+1} B \cap B(w, R) \cap \mathscr{D}(k) \neq \emptyset\right\},
\end{aligned}
$$

Thanks to our choice of $R$, see (41), and the definition of $\mathscr{G}_{\delta}(w, R)$ we have

$$
\frac{R^{h}}{2^{h+1}} \leq \mathcal{S}^{h}\left\llcorner\Gamma(B(w, R)) \leq 2 \mathcal{S}^{h}\left\llcorner\mathscr{D}(k)(B(w, R)) \leq 2 \mathcal{S}^{h}\left\llcorner\mathscr{D}(k)\left(\bigcup_{B \in \mathscr{G}_{\delta}(w, R)} 5^{N+1} B\right) .\right.\right.\right.
$$

Let $\mathscr{G}_{\delta}(w, R):=\left\{B\left(x_{i}, r_{i}\right)\right\}_{i \in \mathbb{N}}$ and recall that $x_{i} \in \mathscr{D}(k)$. This implies, thanks to Corollary 2.8 , that

$$
\begin{aligned}
\mathcal{S}^{h} & \left\llcorner\mathscr{D}(k)\left(\bigcup_{B \in \mathscr{G}_{\delta}(w, R)} 5^{N+1} B\right) \leq 2 \cdot 5^{(N+1) h} \sum_{i \in \mathbb{N}} r_{i}^{h}\right. \\
& =2 \cdot 5^{(N+1) h} C_{2}(\mathbb{V}, \mathbb{L})^{-1} \sum_{i \in \mathbb{N}} \mathcal{S}^{h}\left(P_{\mathbb{V}}\left(B\left(x_{i}, r_{i}\right)\right)\right) \\
& =2 \cdot 5^{(N+1) h} C_{2}(\mathbb{V}, \mathbb{L})^{-1} \mathcal{S}^{h}\left(P_{\mathbb{V}}\left(\bigcup_{i \in \mathbb{N}} B\left(x_{i}, r_{i}\right)\right)\right) \\
& \leq 2 \cdot 5^{(N+1) h} C_{2}(\mathbb{V}, \mathbb{L})^{-1} \mathcal{S}^{h}\left(P_{\mathbb{V}}\left(\bigcup_{B \in \mathscr{F}_{\delta}(w, R)} B\right)\right),
\end{aligned}
$$

where the first inequality comes from the subadditivity of the measure and the upper estimate that we have in the definition of $\mathscr{F}_{\delta}(k)$; while the first identity of the second line above comes from (42). Summing up, for any $\delta>0$ we have

$$
\frac{C_{2}(\mathbb{V}, \mathbb{L}) R^{h}}{5^{(N+1) h} 2^{h+3}} \leq \mathcal{S}^{h}\left(P_{\mathbb{V}}\left(\bigcup_{B \in \mathscr{F} \delta} B, R\right)\right) .
$$

Arguing as in the end of the proof of [4, Proposition 4.6], we get the Hausdorff convergence

$$
P_{\mathbb{V}}\left(\bigcup_{B \in \mathscr{F} \delta} B\right) \underset{H, \delta \rightarrow 0}{\longrightarrow} P_{\mathbb{V}}(\overline{\mathscr{D}(k)} \cap B(w, R)) .
$$

Thanks to the upper semicontinuity of the Lebesgue measure with respect to the Hausdorff convergence we eventually infer that

$$
\begin{aligned}
& \frac{C_{2}(\mathbb{V}, \mathbb{L}) R^{h}}{5^{(N+1) h} 2^{h+3}} \leq \limsup _{\delta \rightarrow 0} \mathcal{S}^{h}\left(P_{\mathbb{V}}\left(\bigcup_{B \in \mathscr{F}_{\delta}(w, R)} B\right)\right) \\
& \quad \leq \mathcal{S}^{h}\left(P_{\mathbb{V}}(\overline{\mathscr{D}(k)} \cap B(w, R))\right) \leq \mathcal{S}^{h}\left(P_{\mathbb{V}}(\Gamma \cap B(w, R))\right),
\end{aligned}
$$

where the last inequality above comes from the compactness of $\Gamma$ and the fact that $\mathscr{D}(k) \subseteq \Gamma$. 
Proposition 3.4 Let us fix $\alpha \leq \varepsilon_{1}(\mathbb{V}, \mathbb{L})$ and suppose $\Gamma$ is a compact $C_{\mathbb{V}}(\alpha)$-set of finite $\mathcal{S}^{h}$-measure such that

$$
\limsup _{r \rightarrow 0} \frac{\mathcal{S}^{h}\llcorner\Gamma(B(x, 2 r))}{\mathcal{S}^{h}\llcorner\Gamma(B(x, r))}<\infty,
$$

for $\mathcal{S}^{h}$-almost every $x \in \Gamma$. Let us set $\varphi: P_{\mathbb{V}}(\Gamma) \rightarrow \mathbb{L}$ the map whose graph is $\Gamma$, see Proposition 2.13, and set $\Phi: P_{\mathbb{V}}(\Gamma) \rightarrow \mathbb{G}$ to be the graph map of $\varphi$. Let us define $\Phi_{*} \mathcal{S}^{h}\llcorner\mathbb{V}$ to be the measure on $\Gamma$ such that for every measurable $A \subseteq \Gamma$ we have $\Phi_{*} \mathcal{S}^{h}\llcorner\mathbb{V}(A):=$ $\mathcal{S}^{h}\left\llcorner\mathbb{V}\left(\Phi^{-1}(A)\right)=\mathcal{S}^{h}\left\llcorner\mathbb{V}\left(P_{\mathbb{V}}(A)\right)\right.\right.$. Then $\Phi_{*} \mathcal{S}^{h}\llcorner\mathbb{V}$ is mutually absolutely continuous with respect to $\mathcal{S}^{h}\llcorner\Gamma$.

Proof The fact that $\Phi_{*} \mathcal{S}^{h}\left\llcorner\mathbb{V}\right.$ is absolutely continuous with respect to $\mathcal{S}^{h}\llcorner\Gamma$ is an immediate consequence of the second part of Corollary 2.8. Viceversa, suppose by contradiction that there exists a compact subset $C$ of $\Gamma$ of positive $\mathcal{S}^{h}$-measure such that

$$
0=\Phi_{*} \mathcal{S}^{h}\left\llcorner\mathbb{V}(C)=\mathcal{S}^{h}\left(P_{\mathbb{V}}(C)\right) .\right.
$$

Since $\mathcal{S}^{h}\llcorner C$ is locally asymptotically doubling by Proposition 2.6 and $C$ has positive and finite $\mathcal{S}^{h}$-measure, we infer thanks to Proposition 3.3 that the set $C$ must have a projection of positive $\mathcal{S}^{h}$-measure. This however comes in contradiction with (43).

In order to prove the forthcoming Proposition 3.6 we need the following result, which is precisely [4, Proposition 4.10].

Proposition 3.5 Suppose $d$ is a homogeneous left-invariant distance on $\mathbb{G}$, let $\mathbb{V}$, $\mathbb{L}$ be complementary subgroups of $\mathbb{G}$ such that $\mathbb{V} \in \mathbb{G}_{c}(h)$, and let us fix $\alpha \leq \varepsilon_{1}(\mathbb{V}, \mathbb{L})$. Suppose that $\Gamma$ is a compact $C_{\mathbb{V}, d}(\alpha)$-set of finite $\mathcal{S}^{h}$-measure. As in Proposition 3.4, let us denote with $\Phi: P_{\mathbb{V}}(\Gamma) \rightarrow \mathbb{G}$ the graph map of $\varphi: P_{\mathbb{V}}(\Gamma) \rightarrow \mathbb{L}$ whose intrinsic graph is $\Gamma$. Then, for $\mathcal{S}_{d}^{h}$-almost every $w \in P_{\mathbb{V}}(\Gamma)$ we have

$$
\lim _{r \rightarrow 0} \frac{\mathcal{S}_{d}^{h}\left(P_{\mathbb{V}}\left(B_{d}(\Phi(w), r) \cap \Phi(w) C_{\mathbb{V}, d}(\alpha)\right) \cap P_{\mathbb{V}}(\Gamma)\right)}{\mathcal{S}_{d}^{h}\left(P_{\mathbb{V}}\left(B_{d}(\Phi(w), r) \cap \Phi(w) C_{\mathbb{V}, d}(\alpha)\right)\right)}=1 .
$$

Proposition 3.6 Let $\alpha \leq \varepsilon_{1}(\mathbb{V}, \mathbb{L})$ and suppose $\Gamma$ is a $C_{\mathbb{V}}(\alpha)$-set such that $\mathcal{S}^{h}\llcorner\Gamma$ is locally asymptotically doubling. Then, $\Theta_{*}^{h}\left(\mathcal{S}^{h}\llcorner\Gamma, x)>0\right.$ for $\mathcal{S}^{h}$-almost every $x \in \Gamma$.

Proof Assume by contradiction that there exists a compact set $C \subseteq \Gamma$ of positive $\mathcal{S}^{h}$-measure such that $\Theta_{*}^{h}\left(\mathcal{S}^{h}\llcorner\Gamma, x)=0\right.$ for every $x \in C$. Since by Proposition 3.4 the measures $\mathcal{S}^{h}\llcorner\Gamma$ and $\Phi_{*} \mathcal{S}^{h}\left\llcorner\mathbb{V}\right.$ are mutually absolutely continuous, the set $P_{\mathbb{V}}(C)$ must have positive $\mathcal{S}^{h}$-measure as well. In particular we have thanks to Proposition 3.5, Lemma 2.14, Corollary 2.8, and Proposition 2.9 that for $\mathcal{S}^{h}$-almost every $x \in C$ we have

$$
\begin{aligned}
\mathcal{S}^{h} & \left(P_{\mathbb{V}}\left(B(0,1) \cap C_{\mathbb{V}}(\alpha)\right)\right) \\
& =\liminf _{r \rightarrow 0} \frac{\mathcal{S}^{h}\left(P_{\mathbb{V}}\left(B(x, \mathfrak{C}(\alpha) r) \cap x C_{\mathbb{V}}(\alpha)\right) \cap P_{\mathbb{V}}(\Gamma)\right)}{\mathcal{S}^{h}\left(P_{\mathbb{V}}\left(B(\Phi(w), \mathfrak{C}(\alpha) r) \cap \Phi(w) C_{\mathbb{V}}(\alpha)\right)\right)} \frac{\mathcal{S}^{h}\left(P_{\mathbb{V}}\left(B(\Phi(w), \mathfrak{C}(\alpha) r) \cap \Phi(w) C_{\mathbb{V}}(\alpha)\right)\right)}{(\mathfrak{C}(\alpha) r)^{h}} \\
& =\liminf _{r \rightarrow 0} \frac{\mathcal{S}^{h}\left(P_{\mathbb{V}}\left(B(x, \mathfrak{C}(\alpha) r) \cap x C_{\mathbb{V}}(\alpha)\right) \cap P_{\mathbb{V}}(\Gamma)\right)}{(\mathfrak{C}(\alpha) r)^{h}} \\
& \leq \liminf _{r \rightarrow 0} \frac{\mathcal{S}^{h}\left\llcorner\mathbb{V}\left(P_{\mathbb{V}}(B(x, r) \cap \Gamma)\right)\right.}{(\mathfrak{C}(\alpha) r)^{h}} \leq 2 C_{1}(\mathbb{V}, \mathbb{L}) \liminf _{r \rightarrow 0} \frac{\mathcal{S}^{h}\llcorner\Gamma(B(x, r))}{(\mathfrak{C}(\alpha) r)^{h}}=0,
\end{aligned}
$$

where $\mathfrak{C}(\alpha)$ is the constant introduced in Lemma 2.14. The above computation is in contradiction with the fact that $\mathcal{S}^{h}\left(P_{\mathbb{V}}\left(B(0,1) \cap C_{\mathbb{V}}(\alpha)\right)\right)$ is positive thus concluding the proof of the proposition. 
We are now in a position to state the main result of this subsection.

Theorem 3.7 Let $\Gamma \subseteq \mathbb{G}$ be compact such that $\mathcal{S}^{h}(\Gamma)<+\infty$. Assume that for $\mathcal{S}^{h}\llcorner\Gamma$-almost every $x \in \mathbb{G}$ we have $\operatorname{Tan}\left(\mathcal{S}^{h}\llcorner\Gamma, x)=\left\{\lambda \mathcal{S}^{h}\llcorner\mathbb{V}(x): \lambda>0\right.\right.$,$\} , where \mathbb{V}(x) \in \mathbb{G}_{c}(h)$. Then, $\mathcal{S}^{h}\left\llcorner\Gamma\right.$ is $\mathscr{P}_{h}^{c}$-rectifiable.

Proof We have that $\mathcal{S}^{h}\llcorner\Gamma$ is locally asymptotically doubling, see Proposition 2.19. Moreover, from Proposition 2.26, there exist $\left\{\mathbb{V}_{i}\right\}_{i \in \mathbb{N}} \subseteq \mathbb{G}_{c}(h)$, and $\left\{\mathbb{L}_{i}\right\}_{i \in \mathbb{N}}$, such that $\mathbb{L}_{i}$ and $\mathbb{V}_{i}$ are homogeneous complementary subgroups, with the property that for every $\alpha>0$ there exists a family of compact sets $\left\{\Gamma_{i}\right\}$ such that $\Gamma_{i}$ is a $C_{\mathbb{V}_{i}}\left(\min \left\{\alpha, \varepsilon_{1}\left(\mathbb{V}_{i}, \mathbb{L}_{i}\right)\right\}\right)$-set, and

$$
\mathcal{S}^{h}\left(\Gamma \backslash \cup_{i \in \mathbb{N}} \Gamma_{i}\right)=0 .
$$

Since $\mathcal{S}^{h}\left\llcorner\Gamma\right.$ is locally asymptotically doubling, then $\mathcal{S}^{h}\left\llcorner\Gamma_{i}\right.$ is locally asymptotically doubling for every $i \in \mathbb{N}$, see Proposition 2.6. Hence, we can apply Proposition 3.6 to conclude that $\Theta_{*}^{h}\left(\mathcal{S}_{\llcorner}^{h} \Gamma_{i}, x\right)>0$ for every $i \in \mathbb{N}$ and for $\mathcal{S}^{h}$-almost every $x \in \Gamma_{i}$. In addition, from the previous inequality and $[15,2.10 .19(5)]$ for every $i \in \mathbb{N}$, we get that

$$
0<\Theta_{*}^{h}\left(\mathcal{S}^{h}\left\llcorner\Gamma_{i}, x\right) \leq \Theta^{h, *}\left(\mathcal{S}^{h}\left\llcorner\Gamma_{i}, x\right)<+\infty, \quad \text { for } \mathcal{S}^{h}-\text { almost every } x \in \Gamma_{i} .\right.\right.
$$

Moreover, since for $\mathcal{S}^{h}$-almost every $x \in \Gamma$ we have $\operatorname{Tan}\left(\mathcal{S}^{h}\llcorner\Gamma, x)=\left\{\lambda \mathcal{S}^{h}\llcorner\mathbb{V}(x): \lambda>0\}\right.\right.$ with $\mathbb{V}(x) \in \mathbb{G}_{c}(h)$, we deduce that, for every $i \in \mathbb{N}$, the locality of tangents in Proposition 2.6 ensures that for $\mathcal{S}^{h}$-almost every $x \in \Gamma_{i}$ we have $\operatorname{Tan}\left(\mathcal{S}^{h}\left\llcorner\Gamma_{i}, x\right)=\left\{\lambda \mathcal{S}^{h}\llcorner\mathbb{V}(x): \lambda>\right.\right.$ $0\}$. From the previous equality, we conclude that for every $i \in \mathbb{N}$ we have $\operatorname{Tan}_{h}(\phi, x) \subseteq$ $\left\{\lambda \mathcal{S}^{h}\llcorner\mathbb{V}(x): \lambda>0\}\right.$. Hence, from the latter conclusion and (46) we get that $\mathcal{S}^{h}\left\llcorner\Gamma_{i}\right.$ is a $\mathscr{P}_{h}^{c}$-rectifiable measure for every $i \in \mathbb{N}$. Finally, from (45) and Proposition 2.6 we conclude that $\mathcal{S}^{h}\left\llcorner\Gamma\right.$ is a $\mathscr{P}_{h}^{c}$-rectifiable measure.

\subsection{From approximate tangent planes to $\mathscr{P}$-rectifiability}

In this section we aim at proving that whenever an approximate (complemented) $h$ dimensional tangent plane to a set $\Gamma$ exists almost everywhere in the sense of Proposition 3.9, then the measure $\mathcal{S}^{h}\left\llcorner\Gamma\right.$ is $\mathscr{P}_{h}^{c}$-rectifiable. First, we need a crucial estimate on projections that will be useful also later on. Since none of the main results of this subsection depends on the choice of the metric, in the following $d$ will be an arbitrary homogeneous left-invariant metric and \|\|$_{d}$ its associated homogeneous norm.

Proposition 3.8 Suppose $d$ is a homogeneous left-invariant distance on $\mathbb{G}$ and let $\mathbb{V}, \mathbb{W} \in$ $\mathbb{G}_{c}(h)$ be complemented by the same subgroup $\mathbb{L}$. Then, there exists an increasing function $\Delta$ : $\left(0, C_{1}(d, \mathbb{W}, \mathbb{L})\right] \rightarrow(0,+\infty)$, depending only on $\mathbb{V}, \mathbb{W}, \mathbb{L}$ and $d$, such that $\lim _{\beta \rightarrow 0} \Delta(\beta)=$ 0 and satisfying the following condition.

For any $\alpha \leq \varepsilon_{1}(\mathbb{V}, \mathbb{L})$ and any $C_{\mathbb{V}, d}(\alpha)$-set $\Gamma$ of finite $\mathcal{S}^{h}$-measure if there are an $x \in \Gamma$, a $\beta \leq C_{1}(d, \mathbb{W}, \mathbb{L})$ and $a \rho>0$ such that

$$
\Gamma \cap B_{d}(x, r) \subseteq x C_{\mathbb{W}, d}(\beta), \quad \text { for all } 0<r<\rho .
$$

then

$$
\left|\frac{\mathcal{S}_{d}^{h}\left(P_{\mathbb{V}}\left(B_{d}(x, r) \cap x C_{\mathbb{W}, d}(\beta)\right) \cap P_{\mathbb{V}}(\Gamma)\right)}{r^{h}}-\frac{\mathcal{S}^{h}\left(P_{\mathbb{V}}\left(B_{d}(x, r) \cap x C_{\mathbb{W}, d}(\beta) \cap \Gamma\right)\right)}{r^{h}}\right| \leq \Delta(\beta),
$$

for any $0<r<\left(1+\alpha\left(C_{1}(d, \mathbb{V}, \mathbb{L})-\alpha\right)^{-1}\right)^{-1} C_{1}(d, \mathbb{V}, \mathbb{L}) \rho=: \varrho(\rho, \alpha)$. 
Proof In order to simplify notation throughout the proof of the proposition, we will drop everywhere the dependence on $d$.

Let us fix an $x \in \Gamma$, a $0<\beta \leq C_{1}(\mathbb{W}, \mathbb{L})$ and a $\rho$ where (47) holds. We denote with $P_{\mathbb{V}}, P_{\mathbb{L}}^{\mathbb{V}}$, respectively, the projections associated to the splitting $\mathbb{G}=\mathbb{V} \cdot \mathbb{L}$, and analogously for the splitting $\mathbb{G}=\mathbb{W} \cdot \mathbb{L}$. For the sake of notation, for any fixed $0<r<\left(1+\alpha\left(C_{1}(\mathbb{V}, \mathbb{L})-\right.\right.$ $\left.\alpha)^{-1}\right)^{-1} C_{1}(\mathbb{V}, \mathbb{L}) \rho$ we let

$$
A_{r}:=P_{\mathbb{V}}\left(B(x, r) \cap x C_{\mathbb{W}}(\beta)\right) \cap P_{\mathbb{V}}(\Gamma) \quad \text { and } \quad B_{r}:=P_{\mathbb{V}}\left(B(x, r) \cap x C_{\mathbb{W}}(\beta) \cap \Gamma\right) .
$$

Since the inclusion $B_{r} \subseteq A_{r}$ is always verified, we want to estimate the measure of those $w$ contained in $A_{r} \backslash B_{r}$. If $y \in A_{r}$, there are $w \in \Gamma$ such that $P_{\mathbb{V}}(w)=y$, and an $\ell \in \mathbb{L}$ such that $y \ell \in B(x, r) \cap x C_{\mathbb{W}}(\beta)$. Let us notice that Corollary 2.8 implies that $\left\|P_{\mathbb{V}}\left(x^{-1} y\right)\right\|=$ $\left\|P_{\mathbb{V}}\left(x^{-1} y \ell\right)\right\| \leq C_{1}(\mathbb{V}, \mathbb{L})^{-1} r$. Moreover, since $\Gamma$ is a $C_{\mathbb{V}}(\alpha)$-set, we even get that, by exploiting Remark 2.2,

$$
\begin{aligned}
\left\|P_{\mathbb{L}}^{\mathbb{V}}\left(x^{-1} w\right)\right\| & \leq \alpha\left(C_{1}(\mathbb{V}, \mathbb{L})-\alpha\right)^{-1}\left\|P_{\mathbb{V}}\left(x^{-1} w\right)\right\|=\alpha\left(C_{1}(\mathbb{V}, \mathbb{L})-\alpha\right)^{-1}\left\|P_{\mathbb{V}}\left(x^{-1} y\right)\right\| \\
& \leq \alpha\left(C_{1}(\mathbb{V}, \mathbb{L})-\alpha\right)^{-1} C_{1}(\mathbb{V}, \mathbb{L})^{-1} r .
\end{aligned}
$$

This implies in particular that

$$
\begin{aligned}
& \left\|x^{-1} w\right\| \leq\left\|P_{\mathbb{V}}\left(x^{-1} w\right)\right\|+\left\|P_{\mathbb{L}}^{\mathbb{V}}\left(x^{-1} w\right)\right\|=\left\|P_{\mathbb{V}}\left(x^{-1} y\right)\right\|+\left\|P_{\mathbb{L}}^{\mathbb{V}}\left(x^{-1} w\right)\right\| \\
& \quad \leq\left(1+\alpha\left(C_{1}(\mathbb{V}, \mathbb{L})-\alpha\right)^{-1}\right) C_{1}^{-1}(\mathbb{V}, \mathbb{L}) r .
\end{aligned}
$$

Hence, from the choice of $r$, we infer that $\left(1+\alpha\left(C_{1}(\mathbb{V}, \mathbb{L})-\alpha\right)^{-1}\right) C_{1}(\mathbb{V}, \mathbb{L})^{-1} r<\rho$ and thus we can use the hypothesis in (47) applied to $w$ to obtain that $x^{-1} w \in C_{\mathbb{W}}(\beta)$. Thus, by also exploiting Remark 2.2 and the fact that $x^{-1} y \ell \in C_{\mathbb{W}}(\beta)$ we get that

$$
\begin{aligned}
& \left\|P_{\mathbb{L}}^{\mathbb{W}}\left(x^{-1} y\right) \ell\right\| \leq \beta\left(C_{1}(\mathbb{W}, \mathbb{L})-\beta\right)^{-1}\left\|P_{\mathbb{W}}\left(x^{-1} y\right)\right\| \text { and } \\
& \left\|P_{\mathbb{L}}^{\mathbb{W}}\left(x^{-1} y\right) P_{\mathbb{L}}^{\mathbb{V}}(w)\right\| \leq \beta\left(C_{1}(\mathbb{W}, \mathbb{L})-\beta\right)^{-1}\left\|P_{\mathbb{W}}\left(x^{-1} y\right)\right\|,
\end{aligned}
$$

where the last inequality comes from the fact that $P_{\mathbb{L}}^{\mathbb{W}}\left(x^{-1} w\right)=P_{\mathbb{L}}^{\mathbb{W}}\left(x^{-1} y P_{\mathbb{L}}^{\mathbb{V}}(w)\right)=$ $P_{\mathbb{L}}^{\mathbb{W}}\left(x^{-1} y\right) P_{\mathbb{L}}^{\mathbb{V}}(w)$. Thanks to (49) we deduce that

$$
\begin{aligned}
\left\|\ell^{-1} P_{\mathbb{L}}^{\mathbb{V}}(w)\right\| & \leq\left\|P_{\mathbb{L}}^{\mathbb{W}}\left(x^{-1} y\right) \ell\right\|+\left\|P_{\mathbb{L}}^{\mathbb{W}}\left(x^{-1} y\right) P_{\mathbb{L}}^{\mathbb{V}}(w)\right\| \\
& \leq 2 \beta\left(C_{1}(\mathbb{W}, \mathbb{L})-\beta\right)^{-1}\left\|P_{\mathbb{W}}\left(x^{-1} y\right)\right\| \\
& =2 \beta\left(C_{1}(\mathbb{W}, \mathbb{L})-\beta\right)^{-1}\left\|P_{\mathbb{W}}\left(x^{-1} y \ell\right)\right\| \\
& \leq 2 \beta\left(C_{1}(\mathbb{W}, \mathbb{L})-\beta\right)^{-1} C_{1}(\mathbb{W}, \mathbb{L})^{-1}\left\|x^{-1} y \ell\right\| \\
& \leq 2 \beta\left(C_{1}(\mathbb{W}, \mathbb{L})-\beta\right)^{-1} C_{1}(\mathbb{W}, \mathbb{L})^{-1} r .
\end{aligned}
$$

This in particular implies that

$\left\|x^{-1} w\right\|=\left\|x^{-1} y P_{\mathbb{L}}^{\mathbb{V}}(w)\right\| \leq r+\left\|\ell^{-1} P_{\mathbb{L}}^{\mathbb{V}}(w)\right\| \leq\left(1+2 \beta\left(C_{1}(\mathbb{W}, \mathbb{L})-\beta\right)^{-1} C_{1}(\mathbb{W}, \mathbb{L})^{-1}\right) r=: f_{2}(\beta) r$.

The above chain of inequalities, together with the hypothesis (47), allows us to conclude that

$$
A_{r} \subseteq P_{\mathbb{V}}\left(B\left(x, f_{2}(\beta) r\right) \cap x C_{\mathbb{W}}(\beta) \cap \Gamma\right) .
$$

Finally this allows us to infer

$$
\begin{aligned}
& \mathcal{S}^{h}\left(A_{r}\right)-\mathcal{S}^{h}\left(B_{r}\right) \leq \mathcal{S}^{h}\left(P_{\mathbb{V}}\left(B\left(x, f_{2}(\beta) r\right) \cap x C_{\mathbb{W}}(\beta) \cap \Gamma\right) \backslash P_{\mathbb{V}}\left(B(x, r) \cap x C_{\mathbb{W}}(\beta) \cap \Gamma\right)\right) \\
& \quad=\mathcal{S}^{h}\left(P_{\mathbb{V}}\left(B\left(x, f_{2}(\beta) r\right) \backslash B(x, r) \cap x C_{\mathbb{W}}(\beta) \cap \Gamma\right)\right),
\end{aligned}
$$


where the last identity comes from the injectivity of $P_{\mathbb{V}}$ when restricted to $\Gamma$, since $\alpha \leq$ $\varepsilon_{1}(\mathbb{V}, \mathbb{L})$, see Proposition 2.13. Finally, Proposition 2.9 implies

$$
\begin{aligned}
\mathcal{S}^{h}\left(A_{r}\right)-\mathcal{S}^{h}\left(B_{r}\right) & \leq \mathcal{S}^{h}\left(P_{\mathbb{V}}\left(B\left(x, f_{2}(\beta) r\right) \backslash B(x, r) \cap x C_{\mathbb{W}}(\beta)\right)\right) \\
& =\mathcal{S}^{h}\left(P_{\mathbb{V}}\left(B\left(0, f_{2}(\beta)\right) \backslash B(0,1) \cap C_{\mathbb{W}}(\beta)\right)\right) r^{h}=: \Delta(\beta) r^{h} .
\end{aligned}
$$

The function $\Delta$ is easily seen to be increasing and thanks to the continuity from above of the measure, the fact that $\lim _{\beta \rightarrow 0} \Delta(\beta)=0$ immediately follows too since $f_{2}(\beta) \rightarrow 1$ as $\beta \rightarrow 0$.

Proposition 3.9 Suppose $d$ is a homogeneous left-invariant distance on $\mathbb{G}$ and that $\Gamma$ is a Borel set of $\mathcal{C}_{d}^{h}$-finite measure such that at $\mathcal{C}_{d}^{h}$-almost every $x \in \Gamma$ there exists $\mathbb{V}(x) \in \mathbb{G}_{c}(h)$ for which for any $0<\beta<1$ there exists a $\rho(x, \beta)>0$ such that

$$
\Gamma \cap B_{d}(x, \rho(x, \beta)) \subseteq x C_{\mathbb{V}(x), d}(\beta) .
$$

Then, the measure $\mathcal{C}_{d}^{h}\left\llcorner\Gamma\right.$ is $\mathscr{P}_{h}^{c}$-rectifiable. In addition we have that $\Theta_{d}^{h}\left(\mathcal{C}_{d}^{h}\llcorner\Gamma, x)=1\right.$ and $\operatorname{Tan}_{h}\left(\mathcal{C}_{d}^{h}\llcorner\Gamma, x)=\left\{\mathcal{C}_{d}^{h}\llcorner\mathbb{V}(x)\}\right.\right.$ for $\mathcal{C}_{d}^{h}\llcorner\Gamma$-almost every $x$.

Proof In order to simplify notation throughout the proof of the proposition, we will drop everywhere the dependence on $d$. First of all we define the family of sets

$$
\begin{aligned}
\mathscr{F} & :=\left\{\Gamma \subseteq \mathbb { G } : \Gamma \text { is Borel, } \mathcal { S } ^ { h } \left\llcorner\Gamma \text { is } \mathscr { P } _ { h } ^ { c } - \text { rectifiable and } \Theta ^ { h } \left(\mathcal{C}^{h}\llcorner\Gamma, x)\right.\right.\right. \\
& =1 \text { for } \mathcal{S}^{h}\llcorner\Gamma-\text { almost every } x\} .
\end{aligned}
$$

Thanks to [40, Proposition 1.22] we can write $\Gamma$ as $\Gamma=\Gamma^{r} \cup \Gamma^{u}$ where $\Gamma^{r}$ is a Borel set for which there are countable many $\Sigma_{k} \in \mathscr{F}$ such that $\Gamma^{r} \subseteq \cup_{k} \Sigma_{k}$ and $\Gamma^{u}$ is a Borel set such that $\mathcal{S}^{h}(\Gamma \cap \Sigma)=0$ for any $\Sigma \in \mathscr{F}$.

Let us prove that $\Gamma^{r} \in \mathscr{F}$. For any $k \in \mathbb{N}$ we define $\tilde{\Sigma}_{k}:=\Sigma_{k} \backslash \cup_{1 \leq i \leq k-1} \Sigma_{i}$. Thanks to Proposition 2.6 the measures $\mathcal{S}^{h}\left\llcorner\tilde{\Sigma}_{k}\right.$ is still $\mathscr{P}_{h}^{c}$-rectifiable, the $\tilde{\Sigma}_{k}$ are pairwise disjoint and their union still contains $\Gamma^{r}$. Again by Proposition 2.6 we infer that for any $k \in \mathbb{N}$ the measure $\mathcal{S}^{h}\left\llcorner\left(\tilde{\Sigma}_{k} \cap \Gamma^{r}\right)\right.$ is $\mathscr{P}_{h}^{c}$-rectifiable and $\Theta^{h}\left(\mathcal{C}^{h}\left\llcorner\left(\tilde{\Sigma}_{k} \cap \Gamma^{r}\right), x\right)=1\right.$ for $\mathcal{S}^{h}\left\llcorner\left(\tilde{\Sigma}_{k} \cap \Gamma^{r}\right)\right.$-almost every $x$ and this finally implies that

$$
\Theta^{h}\left(\mathcal{C}^{h}\left\llcorner\Gamma^{r}, x\right)=1,\right.
$$

for $\mathcal{S}^{h}\left\llcorner\Gamma^{r}\right.$-almost every $x$. Applying Proposition 2.6 to the measure $\mathcal{S}^{h}\left\llcorner\Gamma^{r}\right.$ and to the Borel set $\tilde{\Sigma}_{k}$ we infer that $\operatorname{Tan}_{h}\left(\mathcal{S}^{h}\left\llcorner\Gamma^{r}, x\right)\right.$ is unique and flat $\mathcal{S}^{h}\left\llcorner\Gamma^{r}\right.$-almost everywhere on $\tilde{\Sigma}_{k}$. Since the $\tilde{\Sigma}_{k}$ countably cover $\Gamma^{r}$ this concludes the proof that $\mathcal{S}^{h}\left\llcorner\Gamma^{r}\right.$ is $\mathscr{P}_{h}^{c}$-rectifiable.

The above argument shows that we can assume by contradiction that $\Gamma$ is compact set of positive and finite $\mathcal{S}^{h}$-measure and that

$$
\mathcal{S}^{h}(\Gamma \cap \Sigma)=0 \text { for any } \Sigma \in \mathscr{F} \text {. }
$$

For any $\eta>0$ we let

$$
\mathbb{G}_{c}^{\eta}(h):=\left\{\mathbb{V} \in \mathbb{G}_{c}(h): \inf _{\mathbb{W} \in \mathbb{G}(h) \backslash \mathbb{G}_{c}(h)} d_{\mathbb{G}}(\mathbb{V}, \mathbb{W}) \geq \eta\right\} \subseteq \mathbb{G}_{c}(h) .
$$

Thanks to Proposition 2.7 it follows that $\mathbb{G}_{c}^{\eta}(h)$ is a closed, thus compact, subset of $\mathbb{G}(h)$. Thanks to Proposition 2.24, for any $\eta>0$ the set $\Gamma \eta:=\{x \in \Gamma:(53)$ holds at $x$ and $\mathbb{V}(x) \in$ $\left.\mathbb{G}_{c}^{\eta}(h)\right\}$ is $\mathcal{S}^{h}$-measurable. In addition to this, since $\mathbb{V}(x)$ belongs $\mathcal{S}^{h}\llcorner\Gamma$-almost everywhere to $\mathbb{G}_{c}(h)$, that is an open set in $\mathbb{G}(h)$, see [4, Proposition 2.17], we have

$$
\mathcal{S}^{h}\left(\Gamma \backslash \bigcup_{\eta \in \mathbb{Q}^{+} \backslash\{0\}} \Gamma^{\eta}\right)=0 .
$$


In particular there exists an $\eta_{0}>0$ such that $\mathcal{S}^{h}\left(\Gamma^{\eta_{0}}\right)>0$. In the following we let $E$ be a compact subset of $\Gamma^{\eta_{0}}$ such that

$$
\mathcal{S}^{h}\left(\Gamma^{\eta_{0}} \backslash E\right)<\mathcal{S}^{h}\left(\Gamma^{\eta_{0}}\right) / 2
$$

Note further that thanks to Proposition 2.12 we have that

$$
m\left(\eta_{0}\right):=\min _{\mathbb{W} \in \mathbb{G}_{c}^{\eta_{0}}(h)} \mathfrak{e}(\mathbb{V})>0 .
$$

Let $\mathscr{D}:=\left\{\mathbb{V}_{j}\right\}_{j \in \mathbb{N}}$ be a countable dense subset of $\mathbb{G}_{c}^{\eta_{0}}(h)$ and for all $j \in \mathbb{N}$ we choose a complement $\mathbb{L}_{j}$ of $\mathbb{V}_{j}$ s.t. $\varepsilon_{1}\left(\mathbb{V}_{j}, \mathbb{L}_{j}\right)>\mathfrak{e}\left(\mathbb{V}_{j}\right) / 2 \geq m\left(\eta_{0}\right) / 2$.

From now on we let $\varepsilon$ be a fixed positive number in $\left(0, m\left(\eta_{0}\right) / 10\right)$ such that

$$
1-3 m\left(\eta_{0}\right)^{-1} \varepsilon\left(1+3 m\left(\eta_{0}\right)^{-2} \varepsilon\right) /\left(m\left(\eta_{0}\right)-\varepsilon\right)>0,
$$

which we can do taking $\varepsilon$ small enough. The previous estimate will play a role later on. For any $p, q \in \mathbb{N}$ we define the set

$$
F(p, q):=\left\{x \in E: B(x, 1 / q) \cap \Gamma \subseteq x C_{\mathbb{V}_{p}}(\varepsilon / 6)\right\},
$$

and we claim that

$$
\mathcal{S}^{h}\left(E \backslash \bigcup_{p, q \in \mathbb{N}} F(p, q)\right)=0 .
$$

By density of the family $\mathscr{D}$ in $\mathbb{G}_{c}^{\eta_{0}}(h)$ and since by construction for any $x \in \Gamma^{\eta_{0}}$ we have $\mathbb{V}(x) \in \mathbb{G}_{c}^{\eta_{0}}(h)$, we deduce that there must exist a plane $\mathbb{V}_{p} \in \mathscr{D}$ such that $d_{\mathbb{G}}\left(\mathbb{V}_{p}, \mathbb{V}(x)\right)<$ $30^{-1} \varepsilon$. This, jointly with [4, Lemma 2.15], implies that

$$
C_{\mathbb{V}(x)}\left(30^{-1} \varepsilon\right) \subseteq C_{\mathbb{V}_{p}}\left(6^{-1} \varepsilon\right) .
$$

Since by definition of $\Gamma^{\eta_{0}},(53)$ holds at every point $x \in E$, we can find a $\rho(x)>0$ such that for any $0<r<\rho(x)$ we have

$$
B(x, r) \cap \Gamma \subseteq x C_{\mathbb{V}(x)}\left(30^{-1} \varepsilon\right) .
$$

In particular, putting together (58) and (59) we infer that for $\mathcal{S}^{h}\llcorner\Gamma$-almost every $x \in E$ there are a $p=p(x)>0$ and a $\rho(x)>0$ such that whenever $0<r<\rho(x)$ we have

$$
B(x, r) \cap \Gamma \subseteq x C_{\mathbb{V}_{p}}\left(6^{-1} \varepsilon\right),
$$

and this concludes the proof of (57). Thanks to [4, Proposition 3.3] and Proposition 2.13, we get that there are countably many $\mathbb{V}_{j} \in \mathbb{G}_{c}^{\eta_{0}}(h)$ complemented by some $\mathbb{L}_{j}$, compact subsets $K_{j}$ of $\mathbb{V}_{j}$ and intrinsic Lipschitz functions $\varphi_{j}: K_{j} \subset \mathbb{V}_{j} \rightarrow \mathbb{L}_{j}$ such that

1. for any $z \in K_{j}$ we have $\Gamma_{j}=\left\{w \varphi_{j}(w): w \in K_{j}\right\} \subseteq z \varphi_{j}(z) C_{\mathbb{V}_{\beth}}(\varepsilon)$, and $\Gamma_{j} \subseteq E$

2. $\mathcal{S}^{h}\left(E \backslash \cup_{j} \Gamma_{j}\right)=0$.

Thanks to [14, Corollary 4.17] we know that $\Theta^{*, h}\left(\mathcal{C}^{h}\llcorner E, x) \leq 1\right.$ for $\mathcal{C}^{h}\llcorner E$-almost every $x$ and now we wish to prove that $\Theta_{*}^{h}\left(\mathcal{C}^{h}\llcorner E, x) \geq 1\right.$ for $\mathcal{C}^{h}\llcorner E$-almost every $x$.

Fix a $j \in \mathbb{N}$, and an $x \in \Gamma_{j}$ such that the conclusion in Proposition 3.5 holds. Notice that such a choice of $x$ can be made in a set of $\mathcal{C}^{h}\left\llcorner\Gamma_{j}\right.$-full measure in $\Gamma_{j}$. Suppose that $r_{k}$ is an infinitesimal sequence such that

$$
\Theta_{*}^{h}\left(\mathcal{C}^{h}\left\llcorner\Gamma_{j}, x\right)=\lim _{k \rightarrow \infty} r_{k}^{-h} \mathcal{C}^{h}\left\llcorner\Gamma_{j}\left(B\left(x, r_{k}\right)\right) .\right.\right.
$$


Thanks to item 1 . above and to Proposition 3.8 one infers that we have that for any $k \in \mathbb{N}$ we get

$$
\begin{aligned}
& \left|\frac{\mathcal{C}^{h}\left(P_{\mathbb{V}_{j}}\left(B\left(x, r_{k}\right) \cap x C_{\mathbb{V}_{j}}(\varepsilon)\right) \cap P_{\mathbb{V}_{j}}\left(\Gamma_{j}\right)\right)}{r_{k}^{h}}-\frac{\mathcal{C}^{h}\left(P_{\mathbb{V}_{j}}\left(B\left(x, r_{k}\right) \cap x C_{\mathbb{V}_{j}}(\varepsilon) \cap \Gamma_{j}\right)\right)}{r_{k}^{h}}\right| \\
& \quad \leq \Delta_{j}(\varepsilon),
\end{aligned}
$$

where $\Delta_{j}$ was introduced in the statement of Proposition 3.8 and depends only on the split $\mathbb{V}_{j} \cdot \mathbb{L}_{j}=\mathbb{G}$. In addition to this, for any $j \in \mathbb{N}$ the definitions of $\varepsilon_{1}(\cdot, \cdot)$ and of $\mathbb{L}_{j}$ imply that

$$
C_{1}\left(\mathbb{V}_{j}, \mathbb{L}_{j}\right)=2 \varepsilon_{1}\left(\mathbb{V}_{j}, \mathbb{L}_{j}\right)>\mathfrak{e}\left(\mathbb{V}_{j}\right) \geq m\left(\eta_{0}\right),
$$

and in turn this means that $\Delta_{j}(\varepsilon)$ can be estimated with

$$
\begin{aligned}
\Delta_{j}(\varepsilon) & =\mathcal{C}^{h}\left(P_{\mathbb{V}_{j}}\left(B\left(0, f_{2}(\varepsilon)\right) \backslash B(0,1) \cap C_{\mathbb{V}_{j}}(\varepsilon)\right)\right) \\
& =\mathcal{C}^{h}\left(P_{\mathbb{V}_{j}}\left(B\left(0,1+2 \varepsilon\left(C_{1}\left(\mathbb{V}_{j}, \mathbb{L}_{j}\right)-\varepsilon\right)^{-1} C_{1}\left(\mathbb{V}_{j}, \mathbb{L}_{j}\right)^{-1}\right) \backslash B(0,1) \cap C_{\mathbb{V}_{j}}(\varepsilon)\right)\right) \\
& \leq \mathcal{C}^{h}\left(P_{\mathbb{V}_{j}}\left(B\left(0,1+3 m\left(\eta_{0}\right)^{-2} \varepsilon\right) \backslash B(0,1) \cap C_{\mathbb{V}_{j}}(\varepsilon)\right)\right),
\end{aligned}
$$

where the last inequality above comes from (61) and the fact that $\varepsilon \in\left(0, m\left(\eta_{0}\right) / 10\right)$. From (60), the invariance properties in Proposition 2.9, the fact that $x \in \Gamma_{j}$ was chosen in such a way that Proposition 3.5 holds, and the homogeneity of $\mathcal{C}^{h}\llcorner\mathbb{V}$, we infer that

$$
\begin{aligned}
& \mathcal{C}^{h}\left(P_{\mathbb{V}_{j}}\left(B(0,1) \cap C_{\mathbb{V}_{j}}(\varepsilon)\right)\right) \\
& \left.\limsup _{k \rightarrow+\infty}\left(\frac{\mathcal{C}^{h}\left(P_{\mathbb{V}_{j}}\left(B\left(x, r_{k}\right) \cap x C_{\mathbb{V}_{j}}(\varepsilon)\right) \cap P_{\mathbb{V}_{j}}\left(\Gamma_{j}\right)\right)}{\mathcal{C}^{h}\left(P_{\mathbb{V}_{j}}\left(B\left(x, r_{k}\right) \cap x C_{\mathbb{V}_{j}}(\varepsilon)\right)\right)}-\frac{\mathcal{C}^{h}\left(P_{\mathbb{V}_{j}}\left(B\left(x, r_{k}\right) \cap x C_{\mathbb{V}_{j}}(\varepsilon) \cap \Gamma_{j}\right)\right.}{\mathcal{C}^{h}\left(P_{\mathbb{V}_{j}}\left(B\left(x, r_{k}\right) \cap x C_{\mathbb{V}_{j}}(\varepsilon)\right)\right)}\right)\right) \\
& \quad=\mathcal{C}^{h}\left(P_{\mathbb{V}_{j}}\left(B(0,1) \cap C_{\mathbb{V}_{j}}(\varepsilon)\right)\right)\left(1-\liminf _{k \rightarrow+\infty} \frac{\mathcal{C}^{h}\left(P_{\mathbb{V}_{j}}\left(B\left(x, r_{k}\right) \cap x C_{\mathbb{V}_{j}}(\varepsilon) \cap \Gamma_{j}\right)\right)}{\mathcal{C}^{h}\left(P_{\mathbb{V}_{j}}\left(B\left(x, r_{k}\right) \cap x C_{\mathbb{V}_{j}}(\varepsilon)\right)\right)}\right) \leq \Delta_{j}(\varepsilon) .
\end{aligned}
$$

This implies that, for every $0 \leq \delta \leq 1 / 100$, up to passing to a non-relabelled subsequence in $k$, we can assume without loss of generality that for any $k \in \mathbb{N}$ we have

$$
\begin{aligned}
& 1-\frac{\mathcal{C}^{h}\left(P_{\mathbb{V}_{j}}\left(B\left(x, r_{k}\right) \cap \Gamma_{j}\right)\right)}{r_{k}^{h} \mathcal{C}^{h}\left(P_{\mathbb{V}_{j}}\left(B(0,1) \cap C_{\mathbb{V}_{j}}(\varepsilon)\right)\right)}=1-\frac{\mathcal{C}^{h}\left(P_{\mathbb{V}_{j}}\left(B\left(x, r_{k}\right) \cap x C_{\mathbb{V}_{j}}(\varepsilon) \cap \Gamma_{j}\right)\right)}{\mathcal{C}^{h}\left(P_{\mathbb{V}_{j}}\left(B\left(x, r_{k}\right) \cap x C_{\mathbb{V}_{j}}(\varepsilon)\right)\right)} \\
& \quad \leq \frac{\delta+\Delta_{j}(\varepsilon)}{\mathcal{C}^{h}\left(P_{\mathbb{V}_{j}}\left(B(0,1) \cap C_{\mathbb{V}_{j}}(\varepsilon)\right)\right)} .
\end{aligned}
$$

Now, let us fix a $k \in \mathbb{N}$ sufficiently large such that $\mid \mathcal{C}^{h}\left\llcorner\Gamma_{j}\left(B\left(x, r_{k}\right)\right) / r_{k}^{h}-\Theta_{*}^{h}\left(\mathcal{C}^{h}\left\llcorner\Gamma_{j}, x\right) \mid \leq\right.\right.$ $\delta$, and let $\Gamma_{j}^{\prime} \subseteq \Gamma_{j}$ be a Borel set such that $\left|\mathcal{C}^{h}\left(B\left(x, r_{k}\right) \cap \Gamma_{j}\right)-\mathcal{C}_{0}^{h}\left(B\left(x, r_{k}\right) \cap \Gamma_{j}^{\prime}\right)\right| \leq \delta r_{k}^{h}$. Finally, we choose a covering with balls $\left\{B\left(y_{\ell}, s_{\ell}\right)\right\}_{\ell \in \mathbb{N}}$ of $\Gamma_{j}^{\prime} \cap B\left(x, r_{k}\right)$, with $y_{\ell} \in \Gamma_{j}^{\prime}$, such that $\left|\sum_{\ell \in \mathbb{N}} s_{\ell}^{h}-\mathcal{C}_{0}^{h}\left(B\left(x, r_{k}\right) \cap \Gamma_{j}^{\prime}\right)\right| \leq \delta r_{k}^{h}$. This implies in particular that

$$
\begin{aligned}
& \left|\mathcal{C}^{h}\left(B\left(x, r_{k}\right) \cap \Gamma_{j}\right)-\sum_{\ell \in \mathbb{N}} s_{\ell}^{h}\right| \\
& \quad \leq\left|\mathcal{C}^{h}\left(B\left(x, r_{k}\right) \cap \Gamma_{j}\right)-\mathcal{C}_{0}^{h}\left(B\left(x, r_{k}\right) \cap \Gamma_{j}^{\prime}\right)\right|+\left|\sum_{\ell \in \mathbb{N}} s_{\ell}^{h}-\mathcal{C}_{0}^{h}\left(B\left(x, r_{k}\right) \cap \Gamma_{j}^{\prime}\right)\right| \leq 2 \delta r_{k}^{h} .
\end{aligned}
$$


The above inequalities together imply in particular that for such a $k \in \mathbb{N}$ we have

$$
\begin{aligned}
1 & -\frac{\delta+\Delta_{j}(\varepsilon)}{\mathcal{C}^{h}\left(P_{\mathbb{V}_{j}}\left(B(0,1) \cap C_{\mathbb{V}_{j}}(\varepsilon)\right)\right)} \leq \frac{\mathcal{C}^{h}\left(P_{\mathbb{V}_{j}}\left(B\left(x, r_{k}\right) \cap \Gamma_{j}\right)\right)}{r_{k}^{h} \mathcal{C}^{h}\left(P_{\mathbb{V}_{j}}\left(B(0,1) \cap C_{\mathbb{V}_{j}}(\varepsilon)\right)\right)} \\
& \leq \frac{\mathcal{C}^{h}\left(P_{\mathbb{V}_{j}}\left(B\left(x, r_{k}\right) \cap \Gamma_{j}^{\prime}\right)\right)+C_{2}\left(\mathbb{V}_{j}, \mathbb{L}_{j}\right) \delta r_{k}^{h}}{r_{k}^{h} \mathcal{C}^{h}\left(P_{\mathbb{V}_{j}}\left(B(0,1) \cap C_{\mathbb{V}_{j}}(\varepsilon)\right)\right)} \\
& \leq \frac{\mathcal{C}^{h}\left(P_{\mathbb{V}_{j}}\left(\bigcup_{\ell \in \mathbb{N}} B\left(y_{\ell}, s_{\ell}\right) \cap y_{\ell} C_{\mathbb{V}_{j}}(\varepsilon)\right)\right)+C_{2} \delta r_{k}^{h}}{r_{k}^{h} \mathcal{C}^{h}\left(P_{\mathbb{V}_{j}}\left(B(0,1) \cap C_{\mathbb{V}_{j}}(\varepsilon)\right)\right)} \\
& \leq r_{k}^{-h} \sum_{\ell \in \mathbb{N}} s_{\ell}^{h}+\frac{C_{2} \delta}{\mathcal{C}^{h}\left(P_{\mathbb{V}_{j}}\left(B(0,1) \cap C_{\mathbb{V}_{j}}(\varepsilon)\right)\right)},
\end{aligned}
$$

where in the second inequality we used

$$
\begin{aligned}
& \mathcal{C}^{h}\left(P_{\mathbb{V}_{j}}\left(B\left(x, r_{k}\right) \cap \Gamma_{j}\right)\right)-\mathcal{C}^{h}\left(P_{\mathbb{V}_{j}}\left(B\left(x, r_{k}\right) \cap \Gamma_{j}^{\prime}\right)\right) \\
& \quad=\mathcal{C}^{h}\left(P_{\mathbb{V}_{j}}\left(B\left(x, r_{k}\right) \cap \Gamma_{j} \backslash B\left(x, r_{k}\right) \cap \Gamma_{j}^{\prime}\right)\right) \\
& \quad \leq C_{2} \mathcal{C}^{h}\left(B\left(x, r_{k}\right) \cap \Gamma_{j} \backslash B\left(x, r_{k}\right) \cap \Gamma_{j}^{\prime}\right) \\
& \quad=C_{2}\left(\mathcal{C}^{h}\left(B\left(x, r_{k}\right) \cap \Gamma_{j}\right)-\mathcal{C}^{h}\left(B\left(x, r_{k}\right) \cap \Gamma_{j}^{\prime}\right)\right) \\
& \quad \leq C_{2}\left(\mathcal{C}^{h}\left(B\left(x, r_{k}\right) \cap \Gamma_{j}\right)-\mathcal{C}_{0}^{h}\left(B\left(x, r_{k}\right) \cap \Gamma_{j}^{\prime}\right)\right) \leq C_{2} \delta r_{k}^{h},
\end{aligned}
$$

that is true taking into account the second part of Corollary 2.8, the fact that $P_{\mathbb{V}_{j}}$ is injective on $\Gamma_{j}$, see Proposition 2.13, and the fact that $\mathcal{S}^{h} \leq \mathcal{C}^{h}$ by definition. Hence putting together (63) and (64) we deduce that, for $k$ large enough,

$$
\begin{gathered}
1-\frac{\left(1+C_{2}\right) \delta+\Delta_{j}(\varepsilon)}{\mathcal{C}^{h}\left(P_{\mathrm{V}_{j}}\left(B(0,1) \cap C_{\mathbb{V}_{j}}(\varepsilon)\right)\right)} \\
\leq r_{k}^{-h} \sum_{\ell \in \mathbb{N}} s_{\ell}^{h} \leq \mathcal{C}^{h}\left(B\left(x, r_{k}\right) \cap \Gamma_{j}\right) / r_{k}^{h}+2 \delta \leq \Theta_{*}^{h}\left(\mathcal{C}^{h}\left\llcorner\Gamma_{j}, x\right)+3 \delta .\right.
\end{gathered}
$$

Thanks to the arbitrariness of $\delta$, this implies that for $\mathcal{C}^{h}\left\llcorner\Gamma_{j}\right.$-almost every $x$ we have, by making use of (62)

$$
\begin{aligned}
1 & -\neg(\varepsilon, j):=1-\frac{\mathcal{C}^{h}\left(P_{\mathbb{V}_{j}}\left(B\left(0,1+3 m\left(\eta_{0}\right)^{-2} \varepsilon\right) \backslash B(0,1) \cap C_{\mathbb{V}_{j}}(\varepsilon)\right)\right)}{\mathcal{C}^{h}\left(P_{\mathbb{V}_{j}}\left(B(0,1) \cap C_{\mathbb{V}_{j}}(\varepsilon)\right)\right)} \\
& \leq 1-\frac{\Delta_{j}(\varepsilon)}{\mathcal{C}^{h}\left(P_{\mathbb{V}_{j}}\left(B(0,1) \cap C_{\mathbb{V}_{j}}(\varepsilon)\right)\right)} \\
& \leq \Theta_{*}^{h}\left(\mathcal{C}^{h}\left\llcorner\Gamma_{j}, x\right) \leq \Theta_{*}^{h}\left(\mathcal{C}^{h}\llcorner E, x) .\right.\right.
\end{aligned}
$$

We now wish to get a bound from above of $\rceil(\varepsilon, j)$ that does not depend on $j$. In order to do this, we first of all let $\rho_{1}(\varepsilon):=1+3 m\left(\eta_{0}\right)^{-2} \varepsilon$ and $\rho_{2}(\varepsilon):=1-3 m\left(\eta_{0}\right)^{-1} \varepsilon \rho_{1}(\varepsilon) /\left(m\left(\eta_{0}\right)-\varepsilon\right)>0$, thanks to (55). We claim that the following inclusion holds

$$
\begin{aligned}
& P_{\mathbb{V}_{j}}\left(B\left(0, \rho_{1}(\varepsilon)\right) \backslash B(0,1) \cap C_{\mathbb{V}_{j}}(\varepsilon)\right) \\
& \quad \subseteq P_{\mathbb{V}_{j}}\left(B\left(0, \rho_{1}(\varepsilon)\right) \cap C_{\mathbb{V}_{j}}(\varepsilon)\right) \backslash P_{\mathbb{V}_{j}}\left(B\left(0, \rho_{2}(\varepsilon)\right) \cap C_{\mathbb{V}_{j}}(\varepsilon)\right) .
\end{aligned}
$$

By definition, if $y \in P_{\mathbb{V}_{j}}\left(B\left(0, \rho_{1}(\varepsilon)\right) \backslash B(0,1) \cap C_{\mathbb{V}_{j}}(\varepsilon)\right)$, there exists an $\ell_{1} \in \mathbb{L}_{j}$ such that $y \ell_{1} \in B\left(0, \rho_{1}(\varepsilon)\right) \backslash B(0,1) \cap C_{\mathbb{V}_{j}}(\varepsilon)$. Notice that if $\ell \in \mathbb{L}_{j}$ is such that $y \ell \in C_{\mathbb{V}_{j}}(\varepsilon)$, by 
Corollary 2.8 we have

$$
m\left(\eta_{0}\right)\|\ell\| \leq C_{1}\left(\mathbb{V}_{j}, \mathbb{L}_{j}\right)\|\ell\| \leq \operatorname{dist}\left(y \ell, \mathbb{V}_{j}\right) \leq \varepsilon\|y \ell\| \leq \varepsilon\|y\|+\varepsilon\|\ell\|,
$$

and then $\|\ell\| \leq \varepsilon\|y\| /\left(m\left(\eta_{0}\right)-\varepsilon\right)$. This implies that in order to prove that $y$ does not belong to $P_{\mathbb{V}_{j}}\left(B\left(0, \rho_{2}(\varepsilon)\right) \cap C_{\mathbb{V}_{j}}(\varepsilon)\right)$ we just need to show that $y \ell \notin B\left(0, \rho_{2}(\varepsilon)\right) \cap C_{\mathbb{V}_{j}}(\varepsilon)$ for any $\ell \in B\left(0, \varepsilon\|y\| /\left(m\left(\eta_{0}\right)-\varepsilon\right)\right) \cap \mathbb{L}_{j}$. This however, follows from the following computation

$$
\|y \ell\| \geq\left\|y \ell_{1}\right\|-\left\|\ell_{1}^{-1} \ell\right\| \geq 1-\frac{2 \varepsilon\|y\|}{m\left(\eta_{0}\right)-\varepsilon}>\rho_{2}(\varepsilon),
$$

where the last inequality comes from the fact that $\left\|\ell_{1}^{-1} \ell\right\| \leq\left\|\ell_{1}^{-1}\right\|+\|\ell\| \leq 2 \varepsilon\|y\| /\left(m\left(\eta_{0}\right)-\right.$ $\varepsilon)$, and $\|y\| \leq C_{1}\left(\mathbb{V}_{j}, \mathbb{L}_{j}\right)^{-1}\left\|y \ell_{1}\right\| \leq m\left(\eta_{0}\right)^{-1} \rho_{1}(\varepsilon)$. This proves (67) and in turn the inequality $\neg(\varepsilon, j) \leq \rho_{1}(\varepsilon)^{h}-\rho_{2}(\varepsilon)^{h}$, by homogeneity of $\mathcal{C}^{h}$. Furthermore, since on the righthand side of the previous inequality we have an expression independent on $j$ we conclude that, by exploiting (66), for $\mathcal{C}^{h}$-almost every $x \in \Gamma_{j}$ we have

$$
1-\left(\rho_{1}(\varepsilon)^{h}-\rho_{2}(\varepsilon)^{h}\right) \leq \Theta_{*}^{h}\left(\mathcal{C}^{h}\llcorner E, x) .\right.
$$

Thanks to the arbitrariness of $j$ and to the fact that $\mathcal{C}^{h}\left(E \backslash \cup_{j} \Gamma_{j}\right)=0$, we deduce that the previous inequality holds for $\mathcal{C}^{h}$-almost every $x \in E$. Since $\varepsilon$ can be chosen arbitrarily small, we conclude that $\Theta_{*}^{h}\left(\mathcal{C}^{h}\llcorner E, x) \geq 1\right.$, and then $\Theta^{h}\left(\mathcal{C}^{h}\llcorner E, x)=1\right.$ for $\mathcal{C}^{h}$-almost every $x \in E$.

Eventually, Proposition 2.2 together with (53) concludes that for $\mathcal{C}^{h}$-almost every $x \in E$ and for any $v \in \operatorname{Tan}_{h}\left(\mathcal{C}^{h}\llcorner E, x)\right.$ the support of $v$ is contained in $\mathbb{V}(x)$. In addition to this, from Proposition 2.4 and Proposition 2.5 we have that for $\mathcal{C}^{h}\llcorner E$-almost every $x \in \mathbb{G}$ we have $\operatorname{Tan}_{h}\left(\mathcal{C}^{h}\llcorner E, x)=\left\{\mathcal{C}^{h}\llcorner\mathbb{V}(x)\}\right.\right.$. This concludes the proof of the fact that $\mathcal{C}^{h}\left\llcorner E\right.$ is $\mathscr{P}_{c^{h}}$ rectifiable and this comes in contradiction with the fact that $E \subseteq \Gamma$ has positive $\mathcal{S}^{h}\llcorner\Gamma$-measure by construction and (54).

Let us now verify that an intrinsically differentiable graph satisfies the hypothesis of Proposition 3.9. First, we recall the definition of intrinsically differentiable graph.

Definition 3.1 (Intrinsically differentiable graph) Let $\mathbb{V}$ and $\mathbb{L}$ be two complementary subgroups of a Carnot group $\mathbb{G}$, with $h:=\operatorname{dim}_{H} \mathbb{V}$. Let $\varphi: A \subseteq \mathbb{V} \rightarrow \mathbb{L}$ be a continuous function with $A$ Borel in $\mathbb{V}$. Let $a_{0} \in A$. We say that $\Gamma:=\operatorname{graph}(\varphi):=\{a \cdot \varphi(a): a \in A\}$ is an $h$-dimensional intrinsically differentiable graph at $w \in \Gamma$ if there exists a homogeneous subgroup $\mathbb{V}(w)$ such that for all $k>0$

$$
\lim _{\lambda \rightarrow \infty} d_{H, \mathbb{G}}\left(\delta_{\lambda}\left(w^{-1} \cdot \Gamma\right) \cap B(0, k), \mathbb{V}(w) \cap B(0, k)\right)=0,
$$

where $d_{H, \mathbb{G}}$ is the Hausdorff distance between closed subsets of $\mathbb{G}$. We will call $\mathbb{V}(w)$ the Hausdorff tangent of $\Gamma$ at $w$.

Lemma 3.10 Let $\varphi: A \subseteq \mathbb{V} \rightarrow \mathbb{L}$ be a map such that $\Gamma:=\operatorname{graph}(\varphi)$ is an intrinsically differentiable graph at $w \in \Gamma$ with tangent $\mathbb{V}(w)$. Then, for every $\beta$ there exists $\rho=\rho(\beta)$ such that

$$
\Gamma \cap B(w, \rho) \subseteq w C_{\mathbb{V}(w)}(\beta)
$$

Proof We first claim that for every $\varepsilon>0$ there exists $r_{0}:=r_{0}(\varepsilon)$ such that

$$
\sup _{p \in \Gamma \cap B(w, r)} \operatorname{dist}(p, w \mathbb{V}(w)) \leq \varepsilon r, \quad \text { for all } 0<r \leq r_{0} .
$$


Indeed, this follows just by taking $k=1$ in the definition (68) and by exploiting the very definition of Hausdorff distance.

Now let us take $\varepsilon \leq \beta / 2$. We claim that $\Gamma \cap B\left(w, r_{0}(\varepsilon)\right) \subseteq w C_{\mathbb{V}(w)}(\beta)$. Indeed, let $p \in \Gamma \cap B\left(w, r_{0}(\varepsilon)\right)$, and $k \geq 1$ be such that $r_{0} 2^{-k}<\left\|w^{-1} \cdot p\right\| \leq r_{0} 2^{-k+1}$. Since $p \in \Gamma \cap B\left(w, r_{0} 2^{-k+1}\right)$, from (69) we get

$$
\operatorname{dist}(p, w \mathbb{V}(w)) \leq \varepsilon r_{0} 2^{-k+1} \leq 2 \varepsilon\left\|w^{-1} \cdot p\right\| \leq \beta\left\|w^{-1} \cdot p\right\|,
$$

thus showing the claim.

We are now ready to give the proof of Theorem 1.1 .

Proof of Theorem 1.1 We prove different implications in separate points.

1. $\Rightarrow 2$. If $\mathcal{S}^{h}\left\llcorner\Gamma\right.$ is $\mathscr{P}_{h}^{c}$-rectifiable, then $\mathcal{S}^{h}\llcorner\Gamma$ is asymptoticall doubling. Hence, by a routine argument (cf. [38, Remark 14.4(3)]) we get that, for $\mathcal{S}^{h}\llcorner\Gamma$-almost every $x \in \mathbb{G}$, every element in $\operatorname{Tan}\left(\mathcal{S}^{h}\llcorner\Gamma, x)\right.$ is a constant multiple of an element of $\operatorname{Tan}_{h}\left(\mathcal{S}^{h}\llcorner\Gamma, x)\right.$, which is by hypothesis of the form $\lambda \mathcal{S}^{h}\left\llcorner\mathbb{V}(x)\right.$ with $\mathbb{V}(x) \in \mathbb{G}_{c}(h)$, whence the conclusion.

2 . $\Rightarrow 1$. It follows from Theorem 3.7 by approximating the Borel set $\Gamma$ from within by compact sets.

1. $\Rightarrow 3$. It is a consequence of [4, Theorem 1.8], Proposition 2.26, and the fact that the Hausdorff tangent at $\mathcal{S}^{h}\left\llcorner\Gamma_{i}\right.$-almost ever $x$ of $\Gamma_{i}$ is complemented since it coincides almost everywhere with the subgroup on which it is supported the tangent measure.

3. $\Rightarrow 1$. Since, for every $i \in \mathbb{N}, \Gamma_{i}$ is an intrinsically differentiable graph at $\mathcal{S}^{h}\left\llcorner\Gamma_{i}\right.$-almost every point of it, by Proposition 3.10 we conclude that the hypothesis of Proposition 3.9 is verified. Hence, for every $i \in \mathbb{N}, \mathcal{S}^{h}\left\llcorner\Gamma_{i}\right.$ is $\mathscr{P}_{h}^{c}$-rectifiable. Hence, since $\mathcal{S}^{h}\left(\Gamma \backslash \cup_{i=1}^{+\infty} \Gamma_{i}\right)=0$, by a routine argument involving the locality of tangents and the Lebesgue differentiation theorem, see Proposition 2.6, we conclude that $\mathcal{S}^{h}\left\llcorner\Gamma\right.$ is $\mathscr{P}_{h}^{c}$-rectifiable as well.

Let us show that the item 3. implies the last part of the statement. Since, for every $i \in \mathbb{N}$, $\Gamma_{i}$ is intrinsically differentiable, arguing as above we can apply Proposition 3.9 and then conclude that $\Theta^{h}\left(\mathcal{C}^{h}\left\llcorner\Gamma_{i}, x\right)=1\right.$ for $\mathcal{C}^{h}\left\llcorner\Gamma_{i}\right.$-almost every $x \in \mathbb{G}$. Hence, from the Lebesgue differentiation theorem in Proposition 2.6, we conclude that, for every $i \in \mathbb{N}, \Theta^{h}\left(\mathcal{C}^{h}\llcorner\Gamma, x)=\right.$ 1 for $\mathcal{C}^{h}\left\llcorner\Gamma_{i}\right.$-almost every $x \in \mathbb{G}$, and hence the same conclusion holds for $\mathcal{C}^{h}\llcorner\Gamma$-almost every $x \in \mathbb{G}$ since $\mathcal{C}^{h}\left(\Gamma \backslash \cup_{i=1}^{+\infty} \Gamma_{i}\right)=0$. The last convergence result is a direct consequence of the fact that the density is 1 and [4, Proposition 2.26].

\section{Area formula}

In this section $\mathbb{G}$ is an arbitrary Carnot group, and $\mathbb{V}$ and $\mathbb{L}$ are two homogeneous complementary subgroups, i.e., such that $\mathbb{G}=\mathbb{V} \cdot \mathbb{L}$ and $\mathbb{V} \cap \mathbb{L}=\{0\}$. Moreover, let $h$ be the Hausdorff dimension of $\mathbb{V}$. We equip $\mathbb{G}$ with an arbitrary fixed left-invariant homogeneous distance $d$ that sometimes will be understood.

Lemma 4.1 ([16, Proposition 3.1.5]) Let $\mathbb{V}, \mathbb{L}$ be two complementary subgroups in $\mathbb{G}$. Let $\mathbb{P}$ be a homogeneous subgroup that is a complementary subgroup of $\mathbb{L}$. Then there exists a map $\varphi_{\mathbb{P}}: \mathbb{V} \rightarrow \mathbb{L}$ such that $\mathbb{P}=\Phi_{\mathbb{P}}(\mathbb{V}):=\mathbb{V} \cdot \varphi_{\mathbb{P}}(\mathbb{V})$.

Definition 4.1 (Area factor, [27, Lemma 3.2]) Let $\mathbb{V}, \mathbb{L}$ be two complementary subgroups in $\mathbb{G}$. Let $\mathbb{P}$ be a homogeneous subgroup that is a complementary subgroup of $\mathbb{L}$. Take $\varphi_{\mathbb{P}}: \mathbb{V} \rightarrow \mathbb{L}$ as in Lemma 4.1 and let $\Phi_{\mathbb{P}}: v \mapsto v \cdot \varphi_{\mathbb{P}}(v)$ be its graph map. Then the 
centered area factor of $\mathbb{P}$ with respect to the splitting $\mathbb{V} \cdot \mathbb{L}$ is the unique $0<\mathcal{A}(\mathbb{P})<+\infty$ such that

$$
\mathcal{C}^{h}\left\llcorner\mathbb{P}=\mathcal{A}(\mathbb{P})\left(\Phi_{\mathbb{P}}\right)_{*}\left(\mathcal{C}^{h}\llcorner\mathbb{V}) .\right.\right.
$$

Let $\mathbb{P}, \varphi_{\mathbb{P}}$, and $\Phi_{\mathbb{P}}: \mathbb{V} \rightarrow \mathbb{P}$ be as in Definition 4.1. It is readily seen that $\left(\Phi_{\mathbb{P}}\right)_{*}\left(\mathcal{C}^{h}\llcorner\mathbb{V})\right.$ is a Haar measure on $\mathbb{P}$, compare with the beginning of the proof of [27, Lemma 3.2]. From [18, Theorem 3.1] we conclude

$$
\begin{aligned}
\mathcal{A}(\mathbb{P})^{-1} & =\limsup _{r \rightarrow 0} \frac{\left(\left(\Phi_{\mathbb{P}}\right)_{*}\left(\mathcal{C}^{h}\llcorner\mathbb{V})\right)(B(0, r))\right.}{r^{h}}=\limsup _{r \rightarrow 0} \frac{\mathcal{C}^{h}\left(P_{\mathbb{V}}(B(0, r) \cap \mathbb{P})\right)}{r^{h}} \\
& =\mathcal{C}^{h}\left(P_{\mathbb{V}}(B(0,1) \cap \mathbb{P})\right),
\end{aligned}
$$

where in the last equality we used the homogeneity of $\mathcal{C}^{h}\llcorner\mathbb{V}$.

Lemma 4.2 Given the splitting $\mathbb{G}=\mathbb{V} \cdot \mathbb{L}$, the area factor $\mathcal{A}(\cdot)$ is continuous on the set of homogeneous subgroups that have $\mathbb{L}$ as a complementary subgroup.

Proof It directly follows from the explicit expression in (71) together with a simple argument that can be found, e.g., at the end of [27, Proof of Lemma 3.2].

Definition 4.2 (Elementary $\mathscr{P}_{h}$-rectifiable graph) Let $\mathbb{V}, \mathbb{L}$ be two homogeneous complementary subgroups of a Carnot group $\mathbb{G}$, and $\alpha \leq \varepsilon_{1}(\mathbb{V}, \mathbb{L})$. We say that a compact set $\Gamma$ is an $\alpha$-elementary $\mathscr{P}_{h}^{c}$-rectifiable graph with respect to $\mathbb{V}$ and $\mathbb{L}$ if the following four conditions hold

(i) $\Gamma$ is a compact $C_{\mathbb{V}}(\alpha)$-set of $\mathcal{S}^{h}$-finite measure and thus it is the intrinsic graph of a continuous map $\varphi: A \subseteq \mathbb{V} \rightarrow \mathbb{L}$, with $A:=P_{\mathbb{V}}(\Gamma)$, see Proposition 2.13,

(ii) $\mathcal{S}^{h}\left\llcorner\Gamma\right.$ is a $\mathscr{P}_{h}^{c}$-rectifiable measure,

(iii) for $\mathcal{S}^{h}\left\llcorner\Gamma\right.$-almost every $x \in \mathbb{G}$, the subgroup $\tau(\Gamma, x):=\tau\left(\mathcal{S}^{h}\llcorner\Gamma, x)\right.$ defined in Lemma 2.23 is complementary to $\mathbb{L}$,

(iv) The value of $\mathcal{A}(\tau(\Gamma, x))$ is uniformly bounded above for $\mathcal{S}^{h}\llcorner\Gamma$-almost every $x \in \mathbb{G}$, where $\mathcal{A}$ is the centered area factor defined in Definition 4.1.

For the crucial limit result in Proposition 4.4, we need the following adaptation of [4, Proposition 4.10].

Proposition 4.3 Let $\mathbb{V}, \mathbb{L}$ be complementary subgroups of a Carnot group $\mathbb{G}$. Let us fix $\alpha \leq$ $\varepsilon_{1}(\mathbb{V}, \mathbb{L})$ and suppose that $\Gamma$ is a compact $C_{\mathbb{V}}(\alpha)$-set of finite $\mathcal{S}^{h}$-measure. For $\mathcal{S}^{h}\llcorner\Gamma$-almost every $x \in \mathbb{G}$, let $\mathcal{C}_{x}:=C_{\mathbb{V}(x)}\left(\beta_{x}\right)$, for some $\mathbb{V}(x) \in \mathbb{G}_{c}(h)$ that is complemented by $\mathbb{L}$, and some $\beta_{x}>0$. Let us further assume that $\mathcal{A}(\mathbb{V}(x))$, defined with respect to the splitting $\mathbb{G}=\mathbb{V} \cdot \mathbb{L}$ (see Definition 4.1) is uniformly bounded above by a constant $C$ for $\mathcal{S}^{h}\llcorner\Gamma$-almost every $x \in \mathbb{G}$. As in Proposition 3.4, let us denote with $\Phi: P_{\mathbb{V}}(\Gamma) \rightarrow \mathbb{G}$ the graph map of $\varphi: P_{\mathbb{V}}(\Gamma) \rightarrow \mathbb{L}$ whose intrinsic graph is $\Gamma$.

Then for $\mathcal{S}^{h}$-almost every $w \in P_{\mathbb{V}}(\Gamma)$ we have

$$
\lim _{r \rightarrow 0} \frac{\mathcal{S}^{h}\left(P_{\mathbb{V}}\left(B(\Phi(w), r) \cap \Phi(w) \mathcal{C}_{\Phi(w)}\right) \cap P_{\mathbb{V}}(\Gamma)\right)}{\mathcal{S}^{h}\left(P_{\mathbb{V}}\left(B(\Phi(w), r) \cap \Phi(w) \mathcal{C}_{\Phi(w)}\right)\right)}=1 .
$$

Proof The proof is almost identical to the one of [4, Proposition 4.10], and we outline just the main changes. First, the fine covering $S$ is exactly the same of [4, Proposition 4.10], except that in $(\beta)$ one defines $G(w, r):=P_{\mathbb{V}}\left(B(\Phi(w), r) \cap \Phi(w) C_{\mathbb{V}(\Phi(w))}\left(\beta_{\Phi(w)}\right)\right)$ for 
$w \in P_{\mathbb{V}}(\Gamma)$. Thus we need to check [4, Equation (84)] with the newly defined covering $S$. The verification of [4, Equation (84)] for the part of the covering in $(\alpha)$ is precisely the same as in [4]. Moreover, as it is readily seen by how the estimates are made, arguing verbatim as in [4], and by using the same notation therein, we get whenever $w \in P_{\mathbb{V}}(\Gamma)$ and $0<r<r(w)$ is sufficiently small we have

$$
\hat{G}(w, r) \subseteq P_{\mathbb{V}}(B(\Phi(w), 50(A+1) r)) \cup P_{\mathbb{V}}(B(\Phi(w), C(\Gamma) r)),
$$

where $C(\Gamma)$ is a suitable constant depending only on $\Gamma$. Hence whenever $w \in P_{\mathbb{V}}(\Gamma)$ and $0<r<r(w)$ is sufficiently small we have, by exploiting the homogeneity of $\mathcal{C}^{h}$ and the invariance properties in Proposition 2.9,

$$
\begin{aligned}
& \frac{\mathcal{C}^{h}(\hat{G}(w, r))}{\mathcal{C}^{h}(G(w, r))} \leq \frac{\mathcal{C}^{h}\left(P_{\mathbb{V}}(B(0, \max 50(A+1), C(\Gamma)))\right)}{\mathcal{C}^{h}\left(P_{\mathbb{V}}\left(B(0,1) \cap C_{\mathbb{V}(\Phi(w))}\left(\beta_{\Phi(w)}\right)\right)\right)} \\
& \quad \leq C \mathcal{C}^{h}\left(P_{\mathbb{V}}(B(0, \max 50(A+1), C(\Gamma)))\right)
\end{aligned}
$$

where the last inequality is true since

$$
\begin{aligned}
& \mathcal{C}^{h}\left(P_{\mathbb{V}}\left(B(0,1) \cap C_{\mathbb{V}(\Phi(w))}\left(\beta_{\Phi(w)}\right)\right) \geq \mathcal{C}^{h}\left(P_{\mathbb{V}}(B(0,1) \cap \mathbb{V}(\Phi(w)))\right)\right. \\
& \quad=\mathcal{A}(\mathbb{V}(\Phi(w)))^{-1} \geq C^{-1} .
\end{aligned}
$$

Since the ratio of centered Hausdorff measures on $\mathbb{V}$ is the same as the ratio of spherical Hausdorff measures, the previous estimate allows to conclude that [4, Equation (84)] holds true also for $w \in P_{\mathbb{V}}(\Gamma)$ for this newly defined covering $S$ described above. Hence applications of standard differentiation results allow to conclude the proof of (72) as in [4].

Proposition 4.4 Let $\mathbb{V}, \mathbb{L}$ be two complementary subgroups of $\mathbb{G}$ and let $\alpha \leq \varepsilon_{1}(\mathbb{V}, \mathbb{L})$ and. Let $\Gamma$ be an $\alpha$-elementary $\mathscr{P}_{h}^{c}$-rectifiable graph with respect to $\mathbb{V}, \mathbb{L}$, see Definition 4.2. Then for $\mathcal{S}^{h}\llcorner\Gamma$ almost every $x \in \mathbb{G}$, we have the following equality

$$
\lim _{r \rightarrow 0} r^{-h} \mathcal{C}^{h}\left(P_{\mathbb{V}}(B(x, r) \cap \Gamma)\right)=\mathcal{C}^{h}\left(P_{\mathbb{V}}(B(0,1) \cap \tau(\Gamma, x))\right),
$$

where $\tau(\Gamma, x)$ is the tangent plane at $x$ introduced in Lemma 2.23.

Proof Let us notice first that by means of Proposition 2.9 and from the homogeneity of the measure we get that

$$
\mathcal{C}^{h}\left(P_{\mathbb{V}}\left(B(0,1) \cap \delta_{\lambda}\left(x^{-1} \Gamma\right)\right)\right)=\lambda^{h} \mathcal{C}^{h}\left(P_{\mathbb{V}}\left(B\left(x, \lambda^{-1}\right) \cap \Gamma\right)\right),
$$

for every $x \in \Gamma, \lambda>0$. Let us call $\Phi: A \subseteq \mathbb{V} \rightarrow \mathbb{L}$ the graph map of $\varphi$ as in item (i) of Definition 4.2. From Proposition 3.4 we get that the measure $\Phi_{*}\left(\mathcal{C}^{h}\llcorner\mathbb{V})\right.$ is mutually absolutely continuous with respect to $\mathcal{C}^{h}\llcorner\Gamma$. As a consequence, if we fix $\vartheta, \gamma \in \mathbb{N}$, we have that $\mathcal{C}^{h}\llcorner\Gamma$-almost every point $x$ in $E(\vartheta, \gamma)$, see Definition 2.8 , is a point of density one for the measure $\Phi_{*}\left(\mathcal{C}^{h}\llcorner\mathbb{V})\right.$, that is to say for every $\vartheta, \gamma \in \mathbb{N}$ we have that

$$
\begin{aligned}
& \lim _{r \rightarrow 0} \frac{\left(\Phi_{*}\left(\mathcal{C}^{h}\llcorner\mathbb{V})\right)(B(x, r))\right.}{\left(\Phi_{*}\left(\mathcal{C}^{h}\llcorner\mathbb{V})\right)(B(x, r) \cap E(\vartheta, \gamma))\right.} \\
& \quad=\lim _{r \rightarrow 0} \frac{\mathcal{C}^{h}\left(P_{\mathbb{V}}(B(x, r) \cap \Gamma)\right)}{\mathcal{C}^{h}\left(P_{\mathbb{V}}(B(x, r) \cap E(\vartheta, \gamma))\right)}=1, \quad \text { for } \mathcal{C}^{h}\llcorner\Gamma-\text { almost every } x \in E(\vartheta, \gamma) .
\end{aligned}
$$

From the previous equality, Proposition 2.3, identity (74) and the invariance properties of Proposition 2.9, we conclude that it is sufficient to prove that for every $\vartheta, \gamma \in \mathbb{N}$ we have that

$$
\lim _{r \rightarrow 0} \mathcal{C}^{h}\left(P_{\mathbb{V}}\left(B(0,1) \cap \delta_{1 / r}\left(x^{-1} E(\vartheta, \gamma)\right)\right)\right)=\mathcal{C}^{h}\left(P_{\mathbb{V}}(B(0,1) \cap \tau(\Gamma, x))\right),
$$


for $\mathcal{C}^{h}\llcorner\Gamma$-almost every $x \in E(\vartheta, \gamma)$.

From now on we assume $\vartheta, \gamma \in \mathbb{N}$ to be fixed. Thanks to [4, Proposition 3.2] for $\mathcal{C}^{h}\llcorner\Gamma$ almost every $x \in E(\vartheta, \gamma)$ and any $\beta \leq C_{1}(\tau(\Gamma, x), \mathbb{L})$, there exists a $\tilde{\varrho}(x, \beta)$ such that $E(\vartheta, \gamma) \cap B(x, r) \subseteq x C_{\tau(\Gamma, x)}(\beta)$ for very $0<r<\widetilde{\varrho}(x, \beta)$. For such an $x$ and $\beta \leq$ $C_{1}(\tau(\Gamma, x), \mathbb{L})$, note that Proposition 3.8 with the choices $\Gamma=E(\vartheta, \gamma)$ and $\rho=\tilde{\varrho}(x, \beta)$ allows us to infer that

$$
\begin{aligned}
& \left|\frac{\mathcal{C}^{h}\left(P_{\mathbb{V}}\left(B(x, r) \cap x C_{\tau(\Gamma, x)}(\beta)\right) \cap P_{\mathbb{V}}(E(\vartheta, \gamma))\right)}{r^{h}}-\frac{\mathcal{C}^{h}\left(P_{\mathbb{V}}\left(B(x, r) \cap x C_{\tau(\Gamma, x)}(\beta) \cap E(\vartheta, \gamma)\right)\right)}{r^{h}}\right| \\
& \quad \leq \Delta_{x}(\beta),
\end{aligned}
$$

for any $0<r<\varrho(\tilde{\varrho}(x, \beta), \alpha)$. In addition to this, Proposition 4.3 , the homogeneity of $\mathcal{C}^{h}\llcorner\mathbb{V}$, and the invariance properties of Proposition 2.9 imply that, for any $\beta>0$, we get that for $\mathcal{C}^{h}\llcorner\Gamma$-almost every $x \in E(\vartheta, \gamma)$ we have

$$
\begin{aligned}
\lim _{r \rightarrow 0} & \frac{\mathcal{C}^{h}\left(P_{\mathbb{V}}\left(B(x, r) \cap x C_{\tau(\Gamma, x)}(\beta)\right) \cap P_{\mathbb{V}}(E(\vartheta, \gamma))\right)}{r^{h}} \\
= & \lim _{r \rightarrow 0} \frac{\mathcal{C}^{h}\left(P_{\mathbb{V}}\left(B(x, r) \cap x C_{\tau(\Gamma, x)}(\beta)\right) \cap P_{\mathbb{V}}(E(\vartheta, \gamma))\right)}{\mathcal{C}^{h}\left(P_{\mathbb{V}}\left(B(x, r) \cap x C_{\tau(\Gamma, x)}(\beta)\right)\right)} \cdot \frac{\mathcal{C}^{h}\left(P_{\mathbb{V}}\left(B(x, r) \cap x C_{\tau(\Gamma, x)}(\beta)\right)\right)}{r^{h}} \\
= & \mathcal{C}^{h}\left(P_{\mathbb{V}}\left(B(0,1) \cap C_{\tau(\Gamma, x)}(\beta)\right)\right) .
\end{aligned}
$$

Finally, from the continuity of measures, for $\mathcal{C}^{h}\llcorner\Gamma$-almost every $x \in E(\vartheta, \gamma)$ there exists a function $\Delta_{x}^{\prime}(\beta)$ with $\Delta_{x}^{\prime}(\beta) \rightarrow 0$ as $\beta \rightarrow 0$ (pointwise in $x$ ), and

$$
\left|\mathcal{C}^{h}\left(P_{\mathbb{V}}\left(B(0,1) \cap C_{\tau(\Gamma, x)}(\beta)\right)\right)-\mathcal{C}^{h}\left(P_{\mathbb{V}}(B(0,1) \cap \tau(\Gamma, x))\right)\right| \leq \Delta_{x}^{\prime}(\beta), \quad \text { for all } \beta>0 .
$$

Let us define $E_{n}$ as the set of points $x$ in $E(\vartheta, \gamma)$ such that $\tau(\Gamma, x)$ exists and $C_{1}(\tau(\Gamma, x), \mathbb{L})>$ $1 / n$. Obviously $\mathcal{C}^{h}\left(E(\vartheta, \gamma) \backslash \cup_{n=1}^{+\infty} E_{n}\right)=0$. Thus it is sufficient to prove the claim (75) for $\mathcal{C}^{h}\left\llcorner\Gamma\right.$-almost every $x \in E_{n}$. Let us fix $n \in \mathbb{N}$. The above discussion shows that, if we fix $\beta \leq 1 / n$, then for $\mathcal{C}^{h}\left\llcorner\Gamma\right.$-almost every $x \in E_{n}$ we have that (76), (77) and (78) imply

$$
\begin{aligned}
& \limsup _{r \rightarrow 0}\left|\mathcal{C}^{h}\left(P_{\mathbb{V}}\left(B(0,1) \cap \delta_{1 / r}\left(x^{-1} E(\vartheta, \gamma)\right)\right)\right)-\mathcal{C}^{h}\left(P_{\mathbb{V}}(B(0,1) \cap \tau(\Gamma, x))\right)\right| \\
& \leq \Delta_{x}^{\prime}(\beta)+\limsup _{r \rightarrow 0} \mid \mathcal{C}^{h}\left(P_{\mathbb{V}}\left(B(0,1) \cap \delta_{1 / r}\left(x^{-1} E(\vartheta, \gamma)\right)\right)\right) \\
& -\mathcal{C}^{h}\left(P_{\mathbb{V}}\left(B(0,1) \cap C_{\tau(\Gamma, x)}(\beta)\right)\right) \mid \\
& \leq \Delta_{x}^{\prime}(\beta)+\limsup _{r \rightarrow 0} \mid \frac{\mathcal{C}^{h}\left(P_{\mathbb{V}}\left(B(x, r) \cap x C_{\tau(\Gamma, x)}(\beta) \cap E(\vartheta, \gamma)\right)\right)}{r^{h}} \\
& -\frac{\mathcal{C}^{h}\left(P_{\mathbb{V}}\left(B(x, r) \cap x C_{\tau(\Gamma, x)}(\beta)\right) \cap P_{\mathbb{V}}(E(\vartheta, \gamma))\right)}{r^{h}} \mid \\
& +\limsup _{r \rightarrow 0} \mid \frac{\mathcal{C}^{h}\left(P_{\mathbb{V}}\left(B(x, r) \cap x C_{\tau(\Gamma, x)}(\beta)\right) \cap P_{\mathbb{V}}(E(\vartheta, \gamma))\right)}{r^{h}} \\
& -\frac{\mathcal{C}^{h}\left(P_{\mathbb{V}}\left(B(x, r) \cap x C_{\tau(\Gamma, x)}(\beta)\right)\right)}{r^{h}} \mid \\
& \leq \Delta_{x}^{\prime}(\beta)+\Delta_{x}(\beta) \text {. }
\end{aligned}
$$

Thus by taking the intersection of the $\mathcal{C}^{h}\left\llcorner\Gamma\right.$-full measure sets in $E_{n}$ on which the previous inequality holds for $\beta=1 / m$, with $m \geq n$, we get that for $\mathcal{C}^{h}\left\llcorner\Gamma\right.$-almost every $x \in E_{n}$, the previous inequality holds for every $\beta=1 / m$, with $m \geq n$. By fixing an $x$ in such a set of 
full $\mathcal{C}^{h}\left\llcorner\Gamma\right.$-measure in $E_{n}$ and taking $\beta=1 / m$ in (79) and $m \rightarrow+\infty$, we get the claim (75) for $\mathcal{C}^{h}\left\llcorner\Gamma\right.$-almost every $x \in E_{n}$, and thus the proof is concluded.

Proposition 4.5 There exist a family $\mathscr{F}:=\left\{\mathbb{V}_{k}\right\}_{k \in \mathbb{N}} \subseteq \mathbb{G}_{c}(h)$ and $\mathbb{L}_{k}$ complementary subgroups of $\mathbb{V}_{k}$ such that the following holds. If $\phi$ is a $\mathscr{P}_{h}^{c}$-rectifiable measure, there are continuous maps $\varphi_{k}: A_{k} \subseteq \mathbb{V}_{k} \rightarrow \mathbb{L}_{k}$, with $A_{k}$ compact, such that

(i) for every $k \in \mathbb{N}$ we have $\Gamma_{k}:=\operatorname{graph}\left(\varphi_{k}\right)=A_{k} \cdot \varphi_{k}\left(A_{k}\right)$ is an $\alpha_{k}$-elementary $\mathscr{P}_{h^{-}}^{-}$ rectifiable graph with respect to $\mathbb{V}_{k}$ and $\mathbb{L}_{k}$ for some $\alpha_{k}$, see Definition 4.2,

(ii) $\phi\left(\mathbb{G} \backslash \cup_{k \in \mathbb{N}} \Gamma_{k}\right)=0$,

Proof The result in [4, Theorem 3.4] implies that we can find countably many $\mathbb{V}_{k} \in \mathbb{G}_{c}(h)$ complemented by some $\mathbb{L}_{k}$ such that the following holds. If $\phi$ is a $\mathscr{P}_{h}^{c}$-rectifiable measure, then there exist compact sets $\Gamma_{k}$ such that

1. $\phi\left(\mathbb{G} \backslash \cup_{k \in \mathbb{N}} \Gamma_{k}\right)=0$,

2. for any $k \in \mathbb{N}$ the set $\Gamma_{k}$ is a $C_{\mathbb{V}_{\urcorner}}\left(\min \left\{\varepsilon_{1}\left(\mathbb{V}_{k}, \mathbb{L}_{k}\right), \hbar_{\mathbb{G}}\right\}\right)$-set, where $\hbar_{\mathbb{G}}>0$ is the constant in [4, Proposition 2.8].

It is immediate to see that the measures $\phi$ and $\mathcal{C}^{h}$ are mutually absolutely continuous (see, e.g., [4, Proposition 2.6]) and hence by the Lebesgue differentiation theorem and the locality of tangents, cf. Proposition 2.6, the measure $\mathcal{C}^{h}\left\llcorner\Gamma_{k}\right.$ is still a $\mathscr{P}_{h}^{c}$-rectifiable measure. This proves that each $\Gamma_{k}$ verifies the hypothesis (i) and (ii) of Definition 4.2. In order to check (iii) we note that that, from item 2 . above, for $\mathcal{C}^{h}\left\llcorner\Gamma_{k}\right.$-almost every $x$ we have that the tangent $\mathbb{V}(x)$ is contained in $C_{\mathbb{V}_{k}}\left(\min \left\{\varepsilon_{1}\left(\mathbb{V}_{k}, \mathbb{L}_{k}\right), \hbar_{\mathbb{G}}\right\}\right)=: C_{k}$. This implies thanks to [4, Proof of Proposition 2.17] that $\mathbb{V}(x)$ is a complementary subgroup of $\mathbb{L}_{k}$. In order to conclude the proof of item (iv) of Definition 4.2, we must prove that for any $k \in \mathbb{N}$ there exists a constant $C>0$ such that

$$
\mathcal{C}^{h}\left(P_{\mathbb{V}_{k}}(B(0,1) \cap \mathbb{V}(x))\right)^{-1}=: \mathcal{A}(\mathbb{V}(x)) \leq C \quad \text { for } \mathcal{C}^{h}\left\llcorner\Gamma_{k} \text {-almost any } x \in \mathbb{G} .\right.
$$

Since $\mathbb{V}(x) \subseteq C_{k}$ for $\mathcal{C}^{h}\left\llcorner\Gamma_{k}\right.$-almost every $x \in \mathbb{G}$, it is sufficient to show that there exists a constant $c>0$ such that for any $\mathbb{W} \in \mathbb{G}_{c}(h)$ contained in $C_{k}$ we have

$$
\mathcal{C}^{h}\left(P_{\mathbb{V}_{k}}(B(0,1) \cap \mathbb{W})\right) \geq c .
$$

Suppose by contradiction that there exists a sequence of planes $\mathbb{W}_{i} \in \mathbb{G}_{c}(h)$ contained in $C_{k}$ such that

$$
\mathcal{C}^{h}\left(P_{\mathbb{V}_{k}}\left(B(0,1) \cap \mathbb{W}_{i}\right)\right) \leq i^{-1} .
$$

The compactness result in Proposition 2.7 implies that there exists a $\mathbb{W} \in \mathbb{G}(h)$ such that $\lim _{i \rightarrow 0} d_{\mathbb{G}}\left(\mathbb{W}, \mathbb{W}_{i}\right)=0$. Since $\mathbb{W}_{i} \in C_{k}$ for every $i$ we also get $\mathbb{W} \in C_{k}$ and then, since the aperture of the cone $C_{k}$ is smaller than $\min \left\{\varepsilon_{1}\left(\mathbb{V}_{k}, \mathbb{L}_{k}\right), \hbar_{\mathbb{G}}\right\}$, [4, Proof of Proposition 2.17] we have that $\mathbb{L}_{k}$ and $\mathbb{W}$ are complementary subgroups, and thus $\mathbb{W} \in \mathbb{G}_{c}(h)$. Finally Lemma 4.2 implies that $\mathcal{C}^{h}\left(P_{\mathbb{V}_{k}}(B(0,1) \cap \mathbb{W})\right)=0$. This is not possible since the area factor $\mathcal{A}(\mathbb{W})$ relative to the splitting $\mathbb{G}=\mathbb{V}_{k} \cdot \mathbb{L}_{k}$ should be finite, see Definition 4.1.

Theorem 4.6 (Area formula for the centered measure) Let $\mathbb{V}, \mathbb{L}$ be two complementary subgroups of $\mathbb{G}$. Suppose further $\Gamma$ is an $\alpha$-elementary $\mathscr{P}_{h}^{c}$-rectifiable graph with respect to $\mathbb{V}$ and $\mathbb{L}$, see Definition 4.2. Then, for every Borel function $\psi: \Gamma \rightarrow[0,+\infty)$ we have

$$
\int_{\Gamma} \psi(w) d \mathcal{C}^{h}=\int_{A} \psi(a \cdot \varphi(a)) \mathcal{A}(\tau(\Gamma, a \cdot \varphi(a))) d \mathcal{C}^{h}\llcorner\mathbb{V} .
$$

where $\mathcal{A}(\cdot)$ denotes the centered area factor introduced in Definition 4.1. 
Remark 4.1 Note that the above expression is well defined since thanks to Proposition 3.4 the map $a \rightarrow \tau(\Gamma, a \cdot \varphi(a))$ is defined up to $\mathcal{C}^{h}$-null sets on $\mathbb{V}$.

Proof As a first step, let us show that the map $a \mapsto \mathcal{A}(\tau(\Gamma, a \cdot \varphi(a)))=: f(a)$ is $\mathcal{C}^{h}\llcorner\mathbb{V}$ measurable. To do so let us first recall that

1. the map $a \mapsto a \cdot \varphi(a)$ is continuous from $A$ to $\Gamma$,

2. the map $x \mapsto \tau(\Gamma, x)$ sending points of $\Gamma$ into elements of $\mathbb{G}_{c}(h)$ is $\mathcal{C}^{h}\llcorner\Gamma$-measurable thanks to Lemma 2.23 and for $\mathcal{S}^{h}\llcorner\Gamma$-almost every $x \in \Gamma$ the plane $\tau(\Gamma, x)$ is a complementary subgroup of $\mathbb{L}$ thanks to Definition 4.2(iii),

3. thanks to Lemma 4.2, the function $\mathbb{W} \mapsto \mathcal{A}(\mathbb{W})$ is continuous when restricted to those $\mathbb{W} \in \mathbb{G}_{c}(h)$ that are complements of $\mathbb{L}$.

Finally, items 1., 2. and 3. conclude the proof of the $\mathcal{C}^{h}\llcorner\mathbb{V}$-measurability of the function $f$. In addition to this, thanks to Definition 4.2(iv) we know that $f$ is uniformly bounded on $A$ and thus it is an element of $L_{\text {loc }}^{1}(A)$.

We now introduce a measure $\mu$ supported on $\Gamma$ such that for any Borel set $E$ we have

$$
\mu(E)=\int_{P_{\mathbb{V}}(\Gamma \cap E)} f(a) d \mathcal{C}^{h}\llcorner\mathbb{V}(a) .
$$

Since $\mathcal{C}^{h}\left\llcorner\Gamma\right.$ is a $\mathscr{P}_{h}^{c}$-rectifiable measure, it is locally asymptotically doubling and thus Proposition 3.4 implies that $\mu \ll \mathcal{C}^{h}\left\llcorner\Gamma\right.$. Therefore, if we are able to prove that $\Theta^{h, *}(\mu, x)=1$ for $\mu$-almost every $x \in \Gamma$, then [18, Theorem 3.1] concludes the proof.

Let us now proceed and prove that $\Theta^{h, *}(\mu, x)=1$ for $\mu$-almost every $x \in \Gamma$. As a first step, we note that

$$
\begin{aligned}
& \left|r^{-h} \int_{P_{\mathbb{V}}(B(z \cdot \varphi(z), r) \cap \Gamma)}(f(a)-f(z)) d \mathcal{C}^{h}(a)\right| \\
& \quad \leq \frac{\mathcal{C}^{h}\left(P_{\mathbb{V}}(B(0,1))\right)}{\mathcal{C}^{h}\left(P_{\mathbb{V}}(B(z \cdot \varphi(z), r) \cap \Gamma)\right)} \int_{P_{\mathbb{V}}(B(z \cdot \varphi(z), r) \cap \Gamma)}|f(a)-f(z)| d \mathcal{C}^{h}(a),
\end{aligned}
$$

for any $z \in A$ and where, in order to obtain the above inequality, we used the fact that

$$
\mathcal{C}^{h}\left(P_{\mathbb{V}}(B(z \cdot \varphi(z), r) \cap \Gamma)\right) \leq \mathcal{C}^{h}\left(P_{\mathbb{V}}(B(z \cdot \varphi(z), r))\right)=r^{h} \mathcal{C}^{h}\left(P_{\mathbb{V}}(B(0,1)) .\right.
$$

In addition to this, since $\mathcal{C}^{h}\left\llcorner\Gamma\right.$ is supposed to be a $\mathscr{P}_{h}^{c}$-rectifiable measure, we infer by [4, Proposition 4.9] that for any $z \in P_{\mathbb{V}}(\Gamma)$ there exists a $0<\rho(z)<1$ such that the covering relation of $P_{\mathbb{V}}(\Gamma)$

$$
\left.\left\{\left(z, P_{\mathbb{V}}(B(\Phi(z), r) \cap \Gamma)\right): z \in P_{\mathbb{V}}(\Gamma) \text { and } 0<r<\rho(z)\right\}\right\},
$$

is a $\mathcal{C}^{h}\left\llcorner P_{\mathbb{V}}(\Gamma)\right.$-Vitali relation. Hence, since $f \in L_{\text {loc }}^{1}(A)$, [15, Corollary 2.9.9] allows to conclude that

$$
\lim _{\varepsilon \rightarrow 0^{+}} \sup \left\{\frac{\int_{P_{\mathbb{V}}(B(z \cdot \varphi(z), r) \cap \Gamma)}|f-f(z)| d \mathcal{C}^{h}}{\mathcal{C}^{h}\left(P_{\mathbb{V}}(B(z \cdot \varphi(z), r) \cap \Gamma)\right)}: r<\rho(z), \operatorname{diam}\left(P_{\mathbb{V}}(B(z \cdot \varphi(z), r) \cap \Gamma)\right)<\varepsilon\right\}=0,
$$

for $\mathcal{C}^{h}\left\llcorner P_{\mathbb{V}}(\Gamma)\right.$-almost every $z \in P_{\mathbb{V}}(\Gamma)$. As a consequence, thanks to (81) and (82) we get

$$
\begin{aligned}
& \underset{\mathfrak{r} \rightarrow 0}{\limsup }\left|\mathfrak{r}^{-h} \mu(B(z \cdot \varphi(z), \mathfrak{r}))-f(z) \mathfrak{r}^{-h} \mathcal{C}^{h}\left(P_{\mathbb{V}}(B(z \cdot \varphi(z), \mathfrak{r}) \cap \Gamma)\right)\right| \\
& \leq \limsup _{\mathfrak{r} \rightarrow 0} \frac{\mathcal{C}^{h}\left(P_{\mathbb{V}}(B(0,1))\right)}{\mathcal{C}^{h}\left(P_{\mathbb{V}}(B(z \cdot \varphi(z), \mathfrak{r}) \cap \Gamma)\right)} \int_{P_{\mathbb{V}}(B(z \cdot \varphi(z), \mathfrak{r}) \cap \Gamma)}|f(a)-f(z)| d \mathcal{C}^{h}(a)=0,
\end{aligned}
$$


for $\mathcal{C}^{h}\left\llcorner P_{\mathbb{V}}(\Gamma)\right.$-almost every $z \in P_{\mathbb{V}}(\Gamma)$. Thanks to the absolute continuity of $\mu$ with respect to $\mathcal{C}^{h}\llcorner\Gamma$, and to (83) we infer that for $\mu$-almost every $x \in \Gamma$ we have

$$
\Theta^{*, h}(\mu, x)=f(x) \limsup _{\mathfrak{r} \rightarrow 0} \mathfrak{r}^{-h} \mathcal{C}^{h}\left\llcorner\mathbb{V}\left(P_{\mathbb{V}}(B(x, \mathfrak{r}) \cap \Gamma)\right)=f(x) \mathcal{C}^{h}\left(P_{\mathbb{V}}(B(0,1) \cap \tau(\Gamma, x))\right)=1,\right.
$$

where the last identity follows from the definition of $f,(71)$ and Proposition 4.4.

Corollary 4.7 For any $\mathscr{P}_{h}^{c}$-rectifiable measure $\phi$ there are countably many $\mathbb{V}_{k} \in \mathbb{G}_{c}(h)$ respectively complemented by some $\mathbb{L}_{k}$, and countably many pairwise disjoint elementary $\mathscr{P}_{h}^{c}$-rectifiable graphs $\Gamma_{k}$ with respect to $\mathbb{V}_{k}$ and $\mathbb{L}_{k}$ such that for every Borel function $\psi: \mathbb{G} \rightarrow[0,+\infty)$ we have

$$
\int \psi(w) d \phi(w)=\sum_{k \in \mathbb{N}} \int \psi(a \cdot \varphi(a)) \Theta^{h}(\phi, x) \mathcal{A}_{k}(\tau(\Gamma, a \cdot \varphi(a))) d \mathcal{C}^{h}\left\llcorner\mathbb{V}_{k},\right.
$$

where $\mathcal{A}_{k}(\cdot)$ denotes the centered area factor with respect to the splitting $\mathbb{G}=\mathbb{V}_{k} \cdot \mathbb{L}_{k}$ introduced in Definition 4.1.

Proof First of all, thanks to [4, Theorem 3.4, Proposition 2.5 and Proposition 2.6] there exists a $\mathcal{C}^{h}$ - $\sigma$-finite Borel set $\Sigma$ on which $\phi$ is supported, and moreover $\phi$ is mutually absolutely continuous with respect to $\mathcal{C}^{h}\llcorner\Sigma$. Moreover, from [4, Theorem 4.13] and [18, Theorem 3.1] we conclude that

$$
\phi=\Theta^{h}(\phi, x) \mathcal{C}^{h}\llcorner\Sigma .
$$

Since clearly $\mathcal{C}^{h}\left\llcorner\Sigma\right.$ is $\mathscr{P}_{c}^{h}$-rectifiable thanks to Proposition 2.6, we infer by Proposition 4.5 that we can find the claimed disjoint $\mathscr{P}_{h}^{c}$-elementary graphs $\Gamma_{k}$ covering $\mathcal{C}^{h}$-almost all $\Sigma$. Applying Theorem 4.6 to each one of the $\Gamma_{k}$ s concludes the proof.

Let us now conclude this section by giving the proof of Theorem 1.2 and Theorem 1.3.

Remark 4.2 We remark that Theorem 4.6 holds as well if we substitute the item (i) in the definition of $\alpha$-elementary $\mathscr{P}_{h}^{c}$-rectifiable graph, see Definition 4.2, with the following item

(i) * $\quad \Gamma$ is the compact graph of an intrinsic Lipschitz function $\varphi: A \subseteq \mathbb{V} \rightarrow \mathbb{L}$, see Definition 2.12.

Indeed, the proof of Theorem 4.6 is ultimately based on Proposition 3.4, the differentiation result in Proposition 3.5, and Proposition 4.4, which are themselves based on Proposition 3.4, the differentiation results Proposition 3.5, Proposition 4.3 and Proposition 3.8. All these latter results do not specifically use the fact that $\Gamma$ is a compact $C_{\mathbb{V}}(\alpha)$-set for $\alpha \leq \varepsilon_{1}(\mathbb{V}, \mathbb{L})$ but just two basic consequences of this: namely, the fact that $\mathbb{L} \cap C_{\mathbb{V}}(\alpha)=\{0\}$, see Lemma 2.11, and the fact that $P_{\mathbb{V}}$ is injective on $\Gamma$, see Proposition 2.13. Since we obviously have, by the very definition of the cone $C_{\mathbb{V}, \mathbb{L}}(\alpha)$, that $\mathbb{L} \cap C_{\mathbb{V}, \mathbb{L}}(\alpha)=\{0\}$ for every $\alpha>0$, and since we also readily have that if $\Gamma$ is an intrinsic Lipschitz graph then $P_{\mathbb{V}}$ is injective on $\Gamma$, we conclude that the same strategy of the proof can be adapted to prove Theorem 4.6 with the above modification of the definition of $\alpha$-elementary $\mathscr{P}_{h}^{c}$-rectifiable graph.

Proof (Proof of Theorem 1.2) First of all, let us notice that we can assume $A$, and hence $\Gamma$, to be compact. Indeed, since $A$ is Borel, $\Gamma$ is Borel, because it is the image of $A$ under the graph map of $\varphi$, which is a continuous injective map. Since $\mathcal{S}^{h}\left\llcorner\Gamma\right.$ is $\mathscr{P}_{h}^{c}$-rectifiable we hence deduce that $\Gamma$ is $\mathcal{S}^{h}$ - $\sigma$-finite, cf. [4, Proposition 2.4 and Proposition 2.5]. Hence we have that there exists an increasing sequence $\left\{E_{i}\right\}_{i \in \mathbb{N}}$ of compact sets such that $\chi_{E_{i}} \rightarrow \chi_{\Gamma}$ holds 
$\mathcal{S}^{h}\left\llcorner\Gamma\right.$-almost everywhere. From (8) we also deduce that $\chi_{P_{\mathrm{V}}\left(E_{i}\right)} \rightarrow \chi_{P_{\mathrm{V}}(\Gamma)}$ holds $\mathcal{S}^{h}\llcorner A$ almost everywhere. Hence if we know the Theorem 1.2 to be true for each $E_{i}$ we are done by monotone convergence.

Hence we now prove Theorem 1.2 assuming $A$, and then $\Gamma$, to be compact. Taking into account Remark 4.2, we observe that the proof of Theorem 1.2 is concluded if we have in addition that $\mathcal{A}(\mathbb{V}(x))$ is uniformly bounded above for $\mathcal{S}^{h}\llcorner\Gamma$-almost every $x \in \mathbb{G}$, since it is the only reamining hypothesis to verify in order to apply the modified version of Theorem 4.6 discussed in Remark 4.2. But since $a \rightarrow \mathcal{A}(\mathbb{V}(a \cdot \varphi(a)))$ is $\mathcal{C}^{h}\llcorner\mathbb{V}$-measurable, see the first part of the proof of Theorem 4.6, we can divide the set $A$ into countably many disjoint measurable subsets where $\mathcal{A}(\cdot)$ is uniformly bounded above. Hence, by approximating each of these countably many pieces from the inside with compact sets as explained at the beginning of this proof, and applying Theorem 4.6 as discussed in Remark 4.2, we conclude by approximation that Theorem 4.6 holds on each piece of the latter disjoint union. Then summing together finishes the proof of Theorem 1.2.

Proof of Theorem 1.3 It is a consequence of Theorem 1.2 and Proposition 3.9, taking into account that the intrinsic differentiability implies the hypothesis of Proposition 3.9 (cf. Proposition 3.10) from which we get that $\mathcal{S}^{h}\left\llcorner\Gamma\right.$ is $\mathscr{P}_{h}^{c}$-rectifiable.

\section{Applications}

In this section we provide some applications of the rectifiability criterion proved in Proposition 3.9, which was at the core of the proof of 3. $\Rightarrow 1$. of Theorem 1.1. Let us first recall the definition of $C_{\mathrm{H}}^{1}$-function.

Definition 5.1 ( $C_{\mathrm{H}}^{1}$-function) Let $\mathbb{G}$ and $\mathbb{G}^{\prime}$ be two Carnot groups endowed with left-invariant homogeneous distances $d$ and $d^{\prime}$, respectively. Let $\Omega \subseteq \mathbb{G}$ be open and let $f: \Omega \rightarrow \mathbb{G}^{\prime}$ be a function. We say that $f$ is Pansu differentiable at $x \in \Omega$ if there exists a homogeneous homomorphism $d f_{x}: \mathbb{G} \rightarrow \mathbb{G}^{\prime}$ such that

$$
\lim _{y \rightarrow x} \frac{d^{\prime}\left(f(x)^{-1} \cdot f(y), d f_{x}\left(x^{-1} \cdot y\right)\right)}{d(x, y)}=0 .
$$

Moreover we say that $f$ is of class $C_{\mathrm{H}}^{1}$ in $\Omega$ if the map $x \mapsto d f_{x}$ is continuous from $\Omega$ to the space of homogeneous homomorphisms from $\mathbb{G}$ to $\mathbb{G}^{\prime}$.

Proposition 5.1 Let B be a Borel set in $\mathbb{G}$ and suppose $\mathbb{H}$ is a Carnot group of homogeneous dimension $\mathcal{Q}^{\prime}$ with $\mathcal{Q} \geq \mathcal{Q}^{\prime}$. Let $f: B \subseteq \mathbb{G} \rightarrow \mathbb{H}$ be a Lipschitz map such that

$$
\operatorname{Ker}(d f(x)) \in \mathbb{G}_{c}(\mathbb{G}) \text { for } \mathcal{S}^{\mathcal{Q}} \text { - almost every } x \in\{z \in B: d f(z) \text { exists and is surjective }\},
$$

where $\mathbb{G}_{c}(\mathbb{G})$ denotes the set of complemented homogeneous subgroups in $\mathbb{G}$. Then, for $\mathcal{S}^{\mathcal{Q}^{\prime}}$. almost every $y \in f(B)$, the following holds. For $\mathcal{S}^{\mathcal{Q}-\mathcal{Q}^{\prime}}$-almost every $x \in f^{-1}(y)$ and any $0<\beta<1$ there exists a $\rho(x, \beta)>0$ such that

$$
f^{-1}(y) \cap B(x, \rho(x, \beta)) \subseteq x C_{\operatorname{Ker}(d f(x))}(\beta) .
$$

In particular the measure $\mathcal{S}^{\mathcal{Q}-\mathcal{Q}^{\prime}}\left\llcorner f^{-1}(y)\right.$ is $\mathscr{P}_{\mathcal{Q}-\mathcal{Q}^{\prime}}^{c}$-rectifiable in $\mathbb{G}$ and

$\operatorname{Tan}_{\mathcal{Q}-\mathcal{Q}^{\prime}}\left(\mathcal{S}^{\mathcal{Q}-\mathcal{Q}^{\prime}}\left\llcorner f^{-1}(y), x\right) \subseteq\left\{\lambda \mathcal{C}^{\mathcal{Q}-\mathcal{Q}^{\prime}}\llcorner\operatorname{Ker}(d f(x)): \lambda>0\}\right.\right.$ for $\mathcal{S}^{\mathcal{Q}-\mathcal{Q}^{\prime}-\text { almost every } x \in f^{-1}(y)}$. 
Proof Without loss of generality we can assume that $B$ is a bounded Borel set. Thanks to [33, Equation (9) and Proposition 1.12], since $B$ is bounded and $f$ is Lipschitz on $B$, one infers that for $\mathcal{S}^{\mathcal{Q}^{\prime}}$-almost every $y \in f(B)$ we have $\mathcal{S}^{\mathcal{Q}-\mathcal{Q}^{\prime}}\left(f^{-1}(y)\right)<\infty$. Moreover, from [33, Theorem 2.6 and Theorem 2.7] we have

$$
\mathcal{S}^{\mathcal{Q}-\mathcal{Q}^{\prime}}\left(f^{-1}(y) \cap N\right)=0, \quad \text { and } \quad \mathcal{S}^{\mathcal{Q}-\mathcal{Q}^{\prime}}\left(f^{-1}(y) \cap N^{\prime}\right)=0,
$$

where $N$ is the $\mathcal{S}^{\mathcal{Q}}$-null set on which the Pansu differential $d f(x)$ does not exists and $N^{\prime}$ is the $\mathcal{S}^{\mathcal{Q}}$-measurable set where $d f(x)$ is not surjective. In addition to this, thanks to (86), we know that

$$
\mathcal{S}^{\mathcal{Q}}\left(\left\{z \in B \backslash\left(N \cup N^{\prime}\right): \operatorname{Ker}(d f(x)) \notin \mathbb{G}_{c}(\mathbb{G})\right\}\right)=0,
$$

and thus, [33, Theorem 2.6] implies that for $\mathcal{S}^{\mathcal{Q}^{\prime}}$-almost every $y \in f(B)$ we have

$$
\mathcal{S}^{\mathcal{Q}-\mathcal{Q}^{\prime}}\left(\left\{z \in f^{-1}(y): d f(z) \text { exists, is surjective and } \operatorname{Ker}(d f(z)) \notin \mathbb{G}_{c}(\mathbb{G})\right\}\right)=0 .
$$

We can further refine the above condition thanks to the following observation. Since for $\mathcal{S}^{\mathcal{Q}^{\prime}}$-almost every $y \in f(B)$ and for $\mathcal{S}^{\mathcal{Q}-\mathcal{Q}^{\prime}}$-almost every $z \in f^{-1}(y)$ the Pansu differential $d f(z)$ exists and is surjective, then by the first homomorphism theorem $\mathbb{G} / \operatorname{Ker}(d f(z)) \cong \mathbb{H}$ and in particular the subgroup $\operatorname{Ker}(d f(z))$ must have homogeneous dimension $\mathcal{Q}-\mathcal{Q}^{\prime}$.

Therefore, throughout the rest of the proof, we fix a $y \in f(B)$ such that $\mathcal{S}^{\mathcal{Q}-\mathcal{Q}^{\prime}}\left(f^{-1}(y)\right)<$ $\infty$, and recall that for $\mathcal{S}^{\mathcal{Q}-\mathcal{Q}^{\prime}}$-almost every $z \in f^{-1}(y)$ the Pansu differential $d f(z)$ exists, is surjective and $\operatorname{Ker}(d f(z)) \in \mathbb{G}_{c}\left(\mathcal{Q}-\mathcal{Q}^{\prime}\right)$.

In order to conclude the proof we show that the hypothesis of Proposition 3.9 is satisfied. Fix a point $x \in f^{-1}(y)$ such that $d f(x)$ exists, is surjective and $\operatorname{Ker}(d f(x)) \in \mathbb{G}_{c}\left(\mathcal{Q}-\mathcal{Q}^{\prime}\right)$. Notice that thanks to what we proved above, such $x$ can be chosen in a set of $\mathcal{S}^{\mathcal{Q}-\mathcal{Q}^{\prime}}$-full measure in $f^{-1}(y)$. Let us note that for any $\varepsilon>0$ there exists an $\eta:=\eta(x, \varepsilon)>0$ such that for any $w \in B(x, \eta)$ we have

$$
d_{\mathbb{H}}\left(f(x) d f(x)\left[x^{-1} w\right], f(w)\right) \leq \varepsilon d_{\mathbb{G}}(w, x) .
$$

This in particular implies that for any $w \in B(x, \eta) \cap f^{-1}(y)$ we have

$$
\begin{aligned}
0 & =d_{\mathbb{H}}(f(w), f(x)) \geq d_{\mathbb{H}}\left(f(x) d f(x)\left[x^{-1} w\right], f(x)\right)-d_{\mathbb{H}}\left(f(x) d f(x)\left[x^{-1} w\right], f(w)\right) \\
& \geq d_{\mathbb{H}}\left(f(x) d f(x)\left[x^{-1} w\right], f(x)\right)-\varepsilon d_{\mathbb{G}}(w, x),
\end{aligned}
$$

implying that

$$
\left\|d f(x)\left[P_{\mathbb{L}(x)}\left(x^{-1} w\right)\right]\right\|_{\mathbb{H}}=\left\|d f(x)\left[x^{-1} w\right]\right\|_{\mathbb{H}} \leq \varepsilon\left\|x^{-1} w\right\|_{\mathbb{G}},
$$

where $\mathbb{L}(x)$ is a complementary subgroup of $\operatorname{Ker}(d f(x))$ and $P_{\mathbb{L}(x)}$ is the splitting projection on $\mathbb{L}(x)$ associated to the split $\operatorname{Ker}(d f(x)) \cdot \mathbb{L}(x)$. Thanks to a standard compactness argument, it is not hard to see that there exists a constant $C(x)>0$ such that $\left\|d f(x)\left[P_{\mathbb{L}(x)}\left(x^{-1} w\right)\right]\right\|_{\mathbb{H}} \geq$ $C(x)\left\|P_{\mathbb{L}(x)}\left(x^{-1} w\right)\right\|_{\mathbb{G}}$ for every $w \in B(x, \eta)$, and thus

$$
\operatorname{dist}_{\mathbb{G}}\left(x^{-1} w, \operatorname{Ker}(d f(x))\right) \leq\left\|P_{\mathbb{L}(x)}\left(x^{-1} w\right)\right\|_{\mathbb{G}} \leq C(x)^{-1} \varepsilon\left\|x^{-1} w\right\|_{\mathbb{G}},
$$

for every $w \in B(x, \eta) \cap f^{-1}(y)$, proving that $f^{-1}(y) \cap B(x, \eta) \subseteq x C_{\operatorname{Ker}(d f(x))}\left(C(x)^{-1} \varepsilon\right) \cap$ $B(x, \eta)$. Since we fall in the hypothesis of Proposition 3.9, the proof of the proposition is achieved.

Before stating the following corollaries of Proposition 5.1, let us recall the definition of $\left(\mathbb{G}, \mathbb{G}^{\prime}\right)$-rectifiable sets. 
Definition $5.2\left(C_{\mathrm{H}^{-}}^{1}\right.$-submanifold) Given an arbitrary Carnot group $\mathbb{G}$, we say that $\Sigma \subseteq \mathbb{G}$ is a $C_{\mathrm{H}^{-}}^{1}$-submanifold of $\mathbb{G}$ if there exists a Carnot group $\mathbb{G}^{\prime}$ such that for every $p \in \Sigma$ there exists an open neighborhood $\Omega$ of $p$ and a function $f \in C_{\mathrm{H}}^{1}\left(\Omega ; \mathbb{G}^{\prime}\right)$ such that

$$
\Sigma \cap \Omega=\{g \in \Omega: f(g)=0\},
$$

and $d f_{p}: \mathbb{G} \rightarrow \mathbb{G}^{\prime}$ is surjective with $\operatorname{Ker}\left(d f_{p}\right)$ complemented. In this case we say that $\Sigma$ is a $C_{\mathrm{H}}^{1}\left(\mathbb{G}, \mathbb{G}^{\prime}\right)$-submanifold.

Definition $5.3\left(\left(\mathbb{G}, \mathbb{G}^{\prime}\right)\right.$-rectifiable set) Given two arbitrary Carnot groups $\mathbb{G}$ and $\mathbb{G}^{\prime}$ of homogeneous dimensions $Q$ and $Q^{\prime}$, respectively, we say that $\Sigma \subseteq \mathbb{G}$ is a $\left(\mathbb{G}, \mathbb{G}^{\prime}\right)$-rectifiable set if there exist countably many subsets $\Sigma_{i}$ of $\mathbb{G}$ that are $C_{\mathrm{H}}^{1}\left(\mathbb{G}, \mathbb{G}^{\prime}\right)$-submanifolds, such that

$$
\mathcal{H}^{Q-Q^{\prime}}\left(\Sigma \backslash \bigcup_{i=1}^{+\infty} \Sigma_{i}\right)=0
$$

Corollary 5.2 Suppose $\mathcal{Q} \geq m$, let $B \subseteq \mathbb{G}$ a Borel subset and $f: B \rightarrow \mathbb{R}^{m}$ be a Lipschitz map such that

$\operatorname{Ker}(d f(x)) \in \mathbb{G}_{c}(\mathbb{G})$ for $\mathcal{S}^{\mathcal{Q}}-$ almost every $x \in\{z \in B: d f(z)$ exists and is surjective $\}$.

Then, $m \leq \operatorname{dim}\left(V_{1}\right)$ and for $\mathcal{S}^{m}$-almost every $y \in f(B)$ the set $f^{-1}(y)$ is $C_{H}^{1}\left(\mathbb{G}, \mathbb{R}^{m}\right)$ rectifiable.

Proof First of all, let us note that Proposition 5.1 immediately implies that for $\mathcal{S}^{m}$-almost every $y \in f(B)$, the measure $\mathcal{S}^{\mathcal{Q}-m}\left\llcorner f^{-1}(y)\right.$ is $\mathscr{P}_{\mathcal{Q}-m}^{c}$-rectifiable.

A necessary step to conclude the proof is to investigate further the structure of $\operatorname{Ker}(d f(x))$ whenever it exists. In order to do so, we fix a point where $d f(x)$ exists and note that for any $v \in \mathbb{G}$ we have $d f(x)\left[\delta_{\lambda}(v)\right]=\lambda d f(x)[v]$ for any $\lambda>0$. Since, thanks to the identification through exp of $\mathbb{G}$ with its Lie algebra, $d f(x)$ can be expressed as a matrix, thus we conclude that for every $v \in V_{j}$ with $2 \leq j \leq \kappa$, where $\kappa$ is the step of the group, we have

$$
d f(x)[v]=\lim _{\lambda \rightarrow 0} \frac{d f(x)\left[\delta_{\lambda}(v)\right]}{\lambda}=\lim _{\lambda \rightarrow 0} \frac{\lambda^{j} d f(x)[v]}{\lambda}=0 .
$$

So, $\operatorname{Ker}(d f(x))$ is a normal subgroup containing $V_{2} \oplus \ldots \oplus V_{\kappa}$ and on the points where $d f(x)$ is surjective we must have

$$
\operatorname{dim}_{\text {hom }}(\operatorname{Ker}(d f(x)))=\mathcal{Q}-m \geq \operatorname{dim}_{\text {hom }}\left(V_{2} \oplus \ldots \oplus V_{k}\right)=Q-\operatorname{dim}\left(V_{1}\right),
$$

proving that we have $m \leq \operatorname{dim}\left(V_{1}\right)$.

Throughout the rest of the proof we fix an $y \in f(B)$ such that $f^{-1}(y)$ is a $\mathscr{P}_{\mathcal{Q}-m^{-}}^{c}$ rectifiable measure. In addition to this, since $f^{-1}(y)$ is closed we may as well assume without loss of generality that $f^{-1}(y)$ is compact with $\mathcal{S}^{\mathcal{Q}-m}$-finite measure since the class of $\mathscr{P}_{\mathcal{Q}-m}^{c}$-rectifiable measures is closed under restriction to a Borel subset by splitting $f^{-1}(y)$ as in [4, Proposition 2.4, Proposition 2.5 and Proposition 2.6].

Thanks to Lemma 2.23 applied to the measure $\mathcal{S}^{\mathcal{Q}-m}\left\llcorner f^{-1}(y)\right.$, and thanks to Proposition 5.1, we know that the map $x \mapsto \operatorname{Ker}(d f(x)) \in \mathbb{G}_{c}(\mathcal{Q}-m)$ is $\mathcal{S}^{\mathcal{Q}-m}\left\llcorner f^{-1}(y)\right.$-measurable. Calling $\left\{e_{i}\right\}_{i=1}^{n}$ a basis of $\mathfrak{g}$, the latter observation in conjunction with Proposition 2.10 yields that for any $i=1, \ldots, n$ the vector fields $x \mapsto \Pi_{\operatorname{Ker}(d f(x))^{\perp}}\left[e_{i}\right]=: v_{i}(x)$ are $\mathcal{S}^{\mathcal{Q}-m}\left\llcorner f^{-1}(y)\right.$ measurable. Moreover, thanks to the above discussion, according to which $\operatorname{Ker}(d f(x)) \supseteq$ $V_{2} \oplus \cdots \oplus V_{\kappa}$, each $v_{i}(x)$ belong $\mathcal{S}^{\mathcal{Q}-m}\left\llcorner f^{-1}(y)\right.$-almost everywhere to $G V_{1}$. 
For any $\varepsilon>0$ thanks to Lusin's theorem and the Borel regularity of the measure $\mathcal{S}^{\mathcal{Q}-m}\left\llcorner f^{-1}(y)\right.$, we can find a compact set $K_{\varepsilon} \subseteq \mathbb{G}$ such that $\mathcal{S}^{\mathcal{Q}-m}\left(f^{-1}(y) \backslash K_{\varepsilon}\right) \leq$ $\varepsilon \mathcal{S}^{\mathcal{Q}-m}\left(f^{-1}(y)\right)$ and the vector fields $v_{i}(x)$ are continuous on $K_{\varepsilon}$ for any $i=1, \ldots, n$. In particular we can split $K_{\varepsilon}$ into a finite partition $\left\{K_{\varepsilon}^{I}: I=\left(i_{1}, \ldots, i_{m}\right) \in\{1, \ldots, n\}^{m}\right\}$ of Borel subsets on which the vector fields $v_{i_{1}}(x), \ldots, v_{i_{m}}(x)$ are a basis for $\operatorname{Ker}(d f(x))^{\perp}$.

In the following we will show that for any choice of $I \in\{1, \ldots, n\}^{m}$ such that $\mathcal{S}^{\mathcal{Q}-m}\left\llcorner f^{-1}(y)\left(K_{\varepsilon}^{I}\right)>0\right.$ and of $\vartheta, \gamma \in \mathbb{N}$, the set $E(\vartheta, \gamma)$ relative to the measure $\mathcal{S}^{\mathcal{Q}-m}\left\llcorner K_{\varepsilon}^{I}\right.$, introduced in Definition 2.8 , can be covered $\mathcal{S}^{\mathcal{Q}-m}$-almost all with countably many $C_{H}^{1}\left(\mathbb{G}, \mathbb{R}^{m}\right)$-rectifiable sets. This would imply that $K_{\varepsilon}^{I}$ can be covered $\mathcal{S}^{\mathcal{Q}-m}$-almost all with $C_{H}^{1}\left(\mathbb{G}, \mathbb{R}^{m}\right)$-rectifiable sets thanks to Proposition 2.3 and thus so can $K_{\varepsilon}$ thanks to the finiteness of the family $I$. Finally, the arbitrariness of $\varepsilon$ would conclude the proof of the proposition.

For any $j=1, \ldots, m$ we let

$$
\rho_{j, \delta}(x):=\sup \left\{\frac{\left|\left\langle v_{i_{j}}(x), \pi_{1}\left(x^{-1} z\right)\right\rangle\right|}{\left\|x^{-1} z\right\|}: z \in E(\vartheta, \gamma) \text { and }\left\|x^{-1} z\right\| \leq \delta\right\},
$$

and we claim that for any $j=1, \ldots, m$ we have $\lim _{\delta \rightarrow 0} \rho_{j, \delta}(x)=0$ for any $x \in E(\vartheta, \gamma)$ at which for any $\beta>0$ there exists a $\rho=\rho(x, \beta)>0$ such that

$$
f^{-1}(y) \cap B(x, \rho) \subseteq x C_{\operatorname{Ker}(d f(x))}(\beta) .
$$

Note that thanks to Proposition 5.1, the above condition (91) is satisfied for $\mathcal{S}^{\mathcal{Q}-m}$-almost every $x \in E(\vartheta, \gamma)$. We remark that the functions $\rho_{j, \delta}$ are measurable for any $i \in \mathbb{N}$ and $\delta>0$. Indeed, on the one hand the function $(x, z) \mapsto\left|\left\langle v_{i_{j}}(x), \pi_{1}\left(x^{-1} z\right)\right\rangle\right| / d(x, z)$ is $\mathcal{S}^{\mathcal{Q}-m}\left\llcorner f^{-1}(y)\right.$-measurable since it is the quotient of two $\mathcal{S}^{\mathcal{Q}-m}\left\llcorner f^{-1}(y)\right.$-measurable functions. On the other, since $\mathbb{G}$ is separable, it is immediate to see that $\rho_{j, \delta}$ can be rewritten as the supremum on $z$ over a countable subset of $E(\vartheta, \gamma) \cap B(x, \delta)$, showing that $\rho_{j, \delta}$ is indeed measurable. Thanks to [40, Proposition 1.5], we know that at any $x \in E(\vartheta, \gamma)$ where (91) is satisfied for some $\beta$ and some $\rho>0$, we have

$$
\frac{\left|\left\langle v_{i_{j}}(x), \pi_{1}\left(x^{-1} z\right)\right\rangle\right|}{\left\|x^{-1} z\right\|}=\frac{\operatorname{dist}\left(x^{-1} z, \mathbb{V}\left(v_{i_{j}}(x)\right)\right)}{\left\|x^{-1} z\right\|} \leq \frac{\operatorname{dist}\left(x^{-1} z, \operatorname{Ker}(d f(x))\right)}{\left\|x^{-1} z\right\|} \leq \beta,
$$

for any $z \in B(x, \rho) \cap f^{-1}(y)$, where $\mathbb{V}\left(v_{i_{j}}(x)\right)$ is the 1-codimensional homogeneous subgroup orthogonal (in the Euclidean sense) to the vector $v_{i_{j}}(x)$, and where the second last inequality above comes from the fact that $\operatorname{Ker}(d f(x))$ is contained in $\mathbb{V}\left(v_{i_{j}}(x)\right)$. The bound (92) together with Proposition 5.1 conclude that $\lim _{\delta \rightarrow 0} \rho_{j, \delta}(x)=0$ for $\mathcal{S}^{Q-m}\llcorner E(\vartheta, \gamma)$ almost every $x \in \mathbb{G}$. Thanks to Severini-Egoroff's theorem for any $\tilde{\varepsilon}>0$ we can find a compact set $\tilde{K}_{\tilde{\varepsilon}}$ inside $E(\vartheta, \gamma)$ such that

1. $\mathcal{S}^{\mathcal{Q}-m}\left(E(\vartheta, \gamma) \backslash \tilde{K}_{\tilde{\varepsilon}}\right) \leq \tilde{\varepsilon} \mathcal{S}^{\mathcal{Q}-m}(E(\vartheta, \gamma))$,

2. $v_{i_{j}}(x)$ is continuous on $\tilde{K}_{\tilde{\varepsilon}}$ for any $j=1, \ldots, m$,

3. $\rho_{j, \delta}$ converges uniformly to 0 on $\tilde{K}_{\tilde{\varepsilon}}$ for any $j=1, \ldots, m$.

Thanks to Whitney extension theorem, see for instance [21, Theorem 5.2], we infer that we can find $m C_{\mathrm{H}^{-}}^{1}$-functions defined on all of $\mathbb{G}$ such that $\left.f_{j, \tilde{\varepsilon}}\right|_{\tilde{K}_{\tilde{\varepsilon}}}=0$ and $\nabla_{\mathbb{H}} f_{j, \tilde{\varepsilon}}(x)=v_{i_{j}}(x)$ for any $x \in \widetilde{K}_{\tilde{\varepsilon}}$. This shows that, thanks to arbitrariness of $\tilde{\varepsilon}$ and to the fact that the $v_{i j}$ 's are independent everywhere on $E(\vartheta, \gamma)$, the set $E(\vartheta, \gamma)$ can be covered $\mathcal{S}^{\mathcal{Q}-m}$-almost all by the 0-level set of countably many $C_{H}^{1}\left(\mathbb{G}, \mathbb{R}^{m}\right)$-maps. Thus the proof is concluded. 
We end this section with some consequences of the previous Corollary 5.2. The first part of the forthcoming corollary follows verbatim from the second part of the proof above; while the second part of the forthcoming corollary is a byproduct of the first part in conjunction with [4, Proposition 6.2].

Corollary 5.3 Let $\mathbb{G}$ be a Carnot group of homogeneous dimension $Q$, and let $1 \leq h \leq Q$ be a natural number. Let $\Gamma \subseteq \mathbb{G}$ be a compact set such that $\mathcal{S}^{h}(\Gamma)<+\infty$. The following are equivalent

1. $\mathcal{S}^{h}\left\llcorner\Gamma\right.$ is a $\mathscr{P}_{h}^{c}$-rectifiable measure, and at $\mathcal{S}^{h}\llcorner\Gamma$-almost every $x \in \mathbb{G}$ the tangent plane is complemented by a horizontal subgroup.

2. $\Gamma$ is $C_{\mathrm{H}}^{1}\left(\mathbb{G}, \mathbb{R}^{Q-h}\right)$-rectifiable.

If any of the previous holds we have $Q-h \leq \operatorname{dim}\left(V_{1}\right)$.

Proof $1 . \Rightarrow 2$. is obtained arguing precisely as in the second part of the proof of Corollary 5.2. 2. $\Rightarrow 1$. is an immediate consequence of [4, Proposition 6.2]

Corollary 5.4 Let $B \subseteq \mathbb{G}$ be a Borel subset. Suppose $f: B \rightarrow \mathbb{R}$ is a Lipschitz map. Then, for $\mathcal{S}^{1}$-almost every $y \in f(B)$ the set $f^{-1}(y)$ is $C_{\mathrm{H}^{-}}^{1}$-rectifiable.

Proof Let us assume that $x$ is point where the Pansu's differential $d f(x)$ exists and is surjective. Thanks to the first homomorphism theorem we know that $\mathbb{G} / \operatorname{Ker}(d f(z)) \cong \mathbb{R}$ and in particular $\operatorname{Ker}(d f(z))$ is a 1-codimensional homogeneous subgroup of $\mathbb{G}$. These subgroups are always complemented, and thus Corollary 5.2 concludes the proof.

Corollary 5.5 Let $B \subseteq \mathbb{G}$ be a Borel subset. Suppose $f: B \rightarrow \mathbb{R}^{m}$ is a Lipschitz function with $\mathcal{Q} \geq m$. Then for $\mathcal{S}^{m}$-almost every $y \in f(B)$ there are $m C_{H}^{1}$-rectifiable sets $\Gamma_{i}(y)$ such that

$$
f^{-1}(y)=\bigcap_{i=1}^{m} \Gamma_{i}(y) .
$$

Proof Since $f(z)=y$ if and only if for any $i=1, \ldots, m$ we have $f_{i}(z)=y_{i}$, the claim immediately follows thanks to Corollary 5.2 and Corollary 5.4.

Acknowledgements The first author is partially supported by the European Research Council (ERC Starting Grant 713998 GeoMeG 'Geometry of Metric Groups') led by Enrico Le Donne. The second author is supported by the Simons Foundation grant 601941, GD.

Funding Open access funding provided by Scuola Normale Superiore within the CRUI-CARE Agreement.

Open Access This article is licensed under a Creative Commons Attribution 4.0 International License, which permits use, sharing, adaptation, distribution and reproduction in any medium or format, as long as you give appropriate credit to the original author(s) and the source, provide a link to the Creative Commons licence, and indicate if changes were made. The images or other third party material in this article are included in the article's Creative Commons licence, unless indicated otherwise in a credit line to the material. If material is not included in the article's Creative Commons licence and your intended use is not permitted by statutory regulation or exceeds the permitted use, you will need to obtain permission directly from the copyright holder. To view a copy of this licence, visit http://creativecommons.org/licenses/by/4.0/.

\section{References}

1. Antonelli, G., Di Donato, D., Don, S., Donne, E. L: Characterizations of uniformly differentiable cohorizontal intrinsic graphs in Carnot groups (2020). Preprint on arXiv, arXiv:2005.11390 
2. Antonelli, G., Le Donne, E.: Pauls rectifiable and purely Pauls unrectifiable smooth hypersurfaces. Nonlinear Anal. 200, 111983 (2020). (ISSN: 0362-546X)

3. Antonelli, G., Merlo, A.: Intrinsically Lipschitz functions with normal target in Carnot groups. Ann. Fennici Math. 46, 571-579 (2021)

4. Antonelli, G., Merlo, A.: On rectifiable measures in Carnot groups: structure theory (2020). Preprint on arXiv, arXiv:2009.13941

5. Bigolin, F., Vittone, D.: Some remarks about parametrizations of intrinsic regular surfaces in the Heisenberg group. Publ. Mat. 54(1), 159-172 (2010)

6. Breuillard, E., Le Donne, E.: On the rate of convergence to the asymptotic cone for nilpotent groups and subFinsler geometry. Proc. Natl. Acad. Sci. USA 110(48), 19220-19226 (2013)

7. Citti, G., Manfredini, M., Pinamonti, A., Serra Cassano, F.: Smooth approximation for intrinsic Lipschitz functions in the Heisenberg group. Calc. Var. Partial Diff. Equ. 49(3-4), 1279-1308 (2014)

8. Cole, D., Pauls, S.: C1-hypersurfaces of the Heisenberg group are N-rectifiable. Houston J. Math. 32(3), 713-724 (2006)

9. Corni, F., Magnani, V.: Area formula for regular submanifolds of low codimension in Heisenberg groups (2021). Preprint on arXiv, arXiv:2002.01433

10. De Lellis, C.: Rectifiable sets, densities and tangent measures. Zurich Lectures in Advanced Mathematics. European Mathematical Society (EMS), Zürich, pp. vi+127 (2008). (ISBN: 978-3-03719-044-9)

11. Di Donato, D., Katrin F., Tuomas O.: Metric rectifiability of H-regular surfaces with Hölder continuous horizontal normal. Preprint on arXiv, arXiv:1906.10215. Accepted for publication at International Mathematical Research Notices

12. Don, S., Le Donne, E., Moisala, T., Vittone, D.: A rectifiability result for finite-perimeter sets in Carnot groups. Preprint on arXiv, arXiv:1912.00493. Accepted for publication at Indiana University Mathematics Journal

13. Don, S.: Vittone, D: Fine properties of functions with bounded variation in Carnot-Carathéodory spaces. J. Math. Anal. Appl. 479(1), 482-530 (2019). (ISSN: 0022-247X)

14. Edgar, G.A.: Centered densities and fractal measures. New York J. Math. 13, 33-87 (2007)

15. Federer, H.: Geometric measure theory. Die Grundlehren der mathematischen Wissenschaften, Band 153. Springer-Verlag New York Inc., New York, pp. xiv+676 (1969)

16. Franchi, B., Marchi, M., Serapioni, R.P.: Differentiability and approximate differentiability for intrinsic Lipschitz functions in Carnot groups and a Rademacher theorem. Anal. Geomet. Metr. Spaces 2(1), 258-281 (2014)

17. Franchi, B., Serapioni, R.P.: Intrinsic Lipschitz graphs within Carnot groups. J. Geomet. Anal. 26(3), 1946-1994 (2016). (ISSN: 1050-6926)

18. Franchi, B., Serapioni, R.P., Serra Cassano, F.: Area formula for centered Hausdorff measures in metric spaces. Nonlinear Anal. 126, 218-233 (2015). (ISSN: 0362-546X)

19. Franchi, B., Serapioni, R., Serra Cassano, F.: Differentiability of intrinsic Lipschitz functions within Heisenberg groups. J. Geomet. Anal. 21(4), 1044-1084 (2011). (ISSN: 1050-6926)

20. Franchi, B., Serapioni, R., Serra Cassano, F.: Intrinsic Lipschitz graphs in Heisenberg groups. J. Nonlinear Convex Anal. 7(3), 423-441 (2006). (ISSN: 1345-4773)

21. Franchi, B., Serapioni, R., Serra Cassano, F.: On the structure of finite perimeter sets in step 2 Carnot groups. J. Geomet. Anal. 13(3), 421-466 (2003). (ISSN: 1050-6926)

22. Franchi, B., Serapioni, R., Serra Cassano, F.: Rectifiability and perimeter in the Heisenberg group. Math. Ann. 321(3), 479-531 (2001). (ISSN: 0025-5831)

23. Franchi, B., Serapioni, R., Serra Cassano, F.: Regular hypersurfaces, intrinsic perimeter and implicit function theorem in Carnot groups. Commun. Anal. Geomet. 11(5), 909-944 (2003). (ISSN: 10198385)

24. Franchi, B., Serapioni, R., Serra Cassano, F.: Regular submanifolds, graphs and area formula in Heisenberg groups. Adv. Math. 211(1), 152-203 (2007). (ISSN: 0001-8708)

25. Heinonen, J., Koskela, P., Shanmugalingam, N., Tyson, J.: Sobolev spaces on metric measure spaces. Vol. 27. New Mathematical Monographs. An approach based on upper gradients. Cambridge University Press, Cambridge, pp. xii+434 (2015). (ISBN: 978-1-107-09234-1)

26. Idu, K. O., Valentino M., Francesco, P.M.: Characterizations of k-rectifiability in homogenous groups. Preprint on arXiv, arXiv:2009.03991. Accepted for publication at Journal of Mathematical Analysis and Applications (2020)

27. Julia, A., Nicolussi Golo, S., Vittone, D: Area of intrinsic graphs and Coarea formula in Carnot groups (2020). Preprint on arXiv, arXiv:2004.02520

28. Julia, A., Nicolussi Golo, S., Vittone, D.: Nowhere differentiable intrinsic Lipschitz graphs. Preprint on arXiv, arXiv:2101.02985. Accepted for publication at Bulletin of the London Mathematical Society (2021) 
29. Le Donne, E.: A primer on Carnot groups: homogenous groups, Carnot-Carathéodory spaces, and regularity of their isometries. Anal. Geomet. Metr. Spaces 5(1), 116-137 (2017)

30. Le Donne, E., Nicolussi Golo, S.: Metric Lie groups admitting dilations. Arkiv för Matematik 59, 125-163 (2021)

31. Le Donne, E., Nicolussi Golo, S.: Regularity properties of spheres in homogeneous groups. Trans. Am. Math. Soc. 370(3), 2057-2084 (2018)

32. Le Donne, E., Terhi, M: Semigenerated step-3 Carnot algebras and applications to sub-Riemannian perimeter. Preprint on arXiv, arXiv:2004.08619. Accepted for publication at Mathematische Zeitschrift

33. Magnani, V.: On a general Coarea inequality and applications. Ann. Acad. Sci. Fenn. Math. 27(1), 121-140 (2002). (ISSN: 1239-629X)

34. Magnani, V.: Towards a theory of area in homogeneous groups. Calc. Var. Partial Diff. Equ. 58(3), 91 (2019). (ISSN: 0944-2669)

35. Magnani, V.: Towards differential calculus in stratified groups. J. Aust. Math. Soc. 95(1), 76-128 (2013). (ISSN: 1446-7887)

36. Magnani, V., Tyson, J., Vittone, D.: On transversal submanifolds and their measure. J. Anal. Math. 125, 319-351 (2015)

37. Magnani, V., Vittone, D.: An intrinsic measure for submanifolds in stratified groups. J. Reine Angew. Math. 619, 203-232 (2008)

38. Mattila, P.: Geometry of sets and measures in Euclidean spaces. Vol. 44. Cambridge Studies in Advanced Mathematics. Fractals and rectifiability. Cambridge University Press, Cambridge, pp. xii+343 (1995). (ISBN: 0-521-46576-1)

39. Mattila, P., Serapioni, R., Cassano, F.S.: Characterizations of intrinsic rectifiability in Heisenberg groups. Ann. Sc. Norm. Super. Pisa Cl. Sci. 9(4), 687-723 (2010). (ISSN: 0391-173X)

40. Merlo, A.: Marstrand-Mattila rectifiability criterion for 1-codimensional measures in Carnot groups (2020). Preprint on arXiv, arXiv:2007.03236

41. Pauls, S.: A notion of rectifiability modeled on Carnot groups. Indiana Univ. Math. J. 53(1), 49-81 (2004)

42. Preiss, D.: Geometry of measures in Rn: distribution, rectifiability, and densities. Ann. Math. 125(3), 537-643 (1987). (ISSN: 0003-486X)

43. Vittone, D.: Lipschitz graphs and currents in Heisenberg groups (2020). Preprint on arXiv, arXiv:2007.14286

44. Vittone, D.: Lipschitz surfaces, perimeter and trace theorems for BV functions in Carnot-Carathéodory spaces. Annali della Scuola Normale Superiore di Pisa-Classe di Scienze 11(4), 939-998 (2012)

Publisher's Note Springer Nature remains neutral with regard to jurisdictional claims in published maps and institutional affiliations. 